\title{
30. NEOGENE PLANKTONIC FORAMINIFERA OF THE INDIAN OCEAN (DSDP, LEG 26)
}

\author{
Esteban Boltovskoy, Museo Argentino de Ciencias Naturales "Bernardino Rivadavia" and \\ Consejo Nacional de Investigaciones Cientificas y Tecnicas, Argentina
}

\section{INTRODUCTION}

This report is the result of a study of the Neogene deposits cored in the Indian Ocean during Leg 26 of the Deep Sea Drilling Project.

The main purposes of the study were: (a) qualitative, and in some degree quantitative, study of the planktonic foraminiferal assemblages found in the holes drilled; (b) .determination of the stratigraphic ranges of the main species; (c) establishment of biostratigraphical zonations in the area under study; (d) correlation of the sites drilled; and (e) where possible, the drawing of ecological paleoclimatological as well as taxonomical conclusions.

Time restrictions in the publication schedule did not permit several aspects of the conclusions drawn to be investigated in detail. For the same reason, unfortunately, no study of benthonic fauna was completed. Hopefully, it will be carried out in the neat future.

In spite of these shortcomings, I hope that the main results of this study are adequately based and correct.

To save space, the following abbreviations are used for the samples cited in text and in the plates explanations. Each sample is characterized by numbers which signify core number, section number of that core, and, set off by commas, depth from the top of the section in centimeters. Thus $3-2,40-42 \mathrm{~cm}$ signifies that this sample was taken from Core 3, Section 2, at a depth of $40-42 \mathrm{~cm}$ from the top of the section. Samples from the core catchers are designated by the number of the core and the letters "CC." Thus, a sample taken from the core catcher material of Core 5 will be marked as $5, \mathrm{CC}$.

\section{MATERIAL AND METHODS}

Leg 26 was started on 6 September 1972, in Durban, South Africa, and ended on 31 October 1972, in Fremantle, Australia. The Glomar Challenger crossed the Indian Ocean mainly in the temperate zone; only two sites were located in the subtropical zone.

During Leg 26, the shipboard paleontologists worked together in determining the age of the sediments cored. At that time practically the only material studied was from core catchers (average volume of each core catcher is about $25-30 \mathrm{cc}$ ). In special, infrequent, cases some additional samples, taken near the biostratigraphic boundaries, were examined also.

In total, nine sites were drilled (Sites 250-258). Six of them (Sites 250,251, 253, 254, 255, 258) gave well- or relatively well-preserved faunas. Three other sites (Sites $252,256,257)$, situated in very deep water, were barren because of dissolution effects.

The list of sites cored is given in Table 1.
TABLE 1

Site Summary

\begin{tabular}{ccrc}
\hline Site & Latitude S & Longitude E & $\begin{array}{c}\text { Water Depth } \\
(\mathrm{m})\end{array}$ \\
\hline 250 & $33^{\circ} 27.74^{\prime}$ & $39^{\circ} 22.15^{\prime}$ & 5119 \\
251 & $36^{\circ} 30.26^{\prime}$ & $49^{\circ} 29.08^{\prime}$ & 3489 \\
252 & $37^{\circ} 02.44^{\prime}$ & $59^{\circ} 14.33^{\prime}$ & 5032 \\
253 & $24^{\circ} 52.65^{\prime}$ & $87^{\circ} 21.97^{\prime}$ & 1962 \\
254 & $30^{\circ} 58.15^{\prime}$ & $87^{\circ} 53.72^{\prime}$ & 1253 \\
255 & $31^{\circ} 07.87^{\prime}$ & $93^{\circ} 43.72^{\prime}$ & 1144 \\
256 & $23^{\circ} 27.35^{\prime}$ & $100^{\circ} 46.46^{\prime}$ & 5361 \\
257 & $30^{\circ} 59.16^{\prime}$ & $108^{\circ} 20.99^{\prime}$ & 5278 \\
258 & $33^{\circ} 47.69^{\prime}$ & $112^{\circ} 28.42^{\prime}$ & 2793 \\
\hline
\end{tabular}

Figure 1 shows the location of the sites studied.

For study in the shore laboratory R. Herb took Cretaceous and Paleogene foraminifera and $\mathrm{E}$. Boltovskoy took Neogene foraminifera. The PaleogeneNeogene transitional zone was sampled by both. The following samples were taken by E. Boltovskoy:

1) One sample per core section and at closer intervals near biostratigraphic boundaries (each sample was about $10 \mathrm{cc}$ ).

2) Samples from those places in cores which showed some lithologic changes.

3) Additional material from core catchers for more detailed study than that completed aboard ship.

The following numbers of Neogene samples were studied from each site.

\begin{tabular}{cr} 
Site 250 & 117 \\
Site 251 & 194 \\
Site 252 & 12 \\
Site 253 & 89 \\
Site 254 & 175 \\
Site 255 & 17 \\
Site 256 & 44 \\
Site 257 & 31 \\
Site 258 & 200 \\
\hline Total & 879
\end{tabular}

All the samples were soaked and, if necessary, treated with hydrogen peroxide, or boiled in a weak solution of washing soda. In some cases, if the sediment was rather consolidated, a mixture of sodium pyrophosphate and $15 \%$ hydrogen peroxide was used.

Then the material was washed over a screen having average mesh of $0.063 \mathrm{~mm}$ (U.S. Sieve Series Mesh no. 230 ). The washed residue was then dried, and from each sample 200-250 foraminiferal tests were picked out at random. The remaining residue was thoroughly examined and all rare specimens, as well as probable indicator species, were also extracted. It was observed 


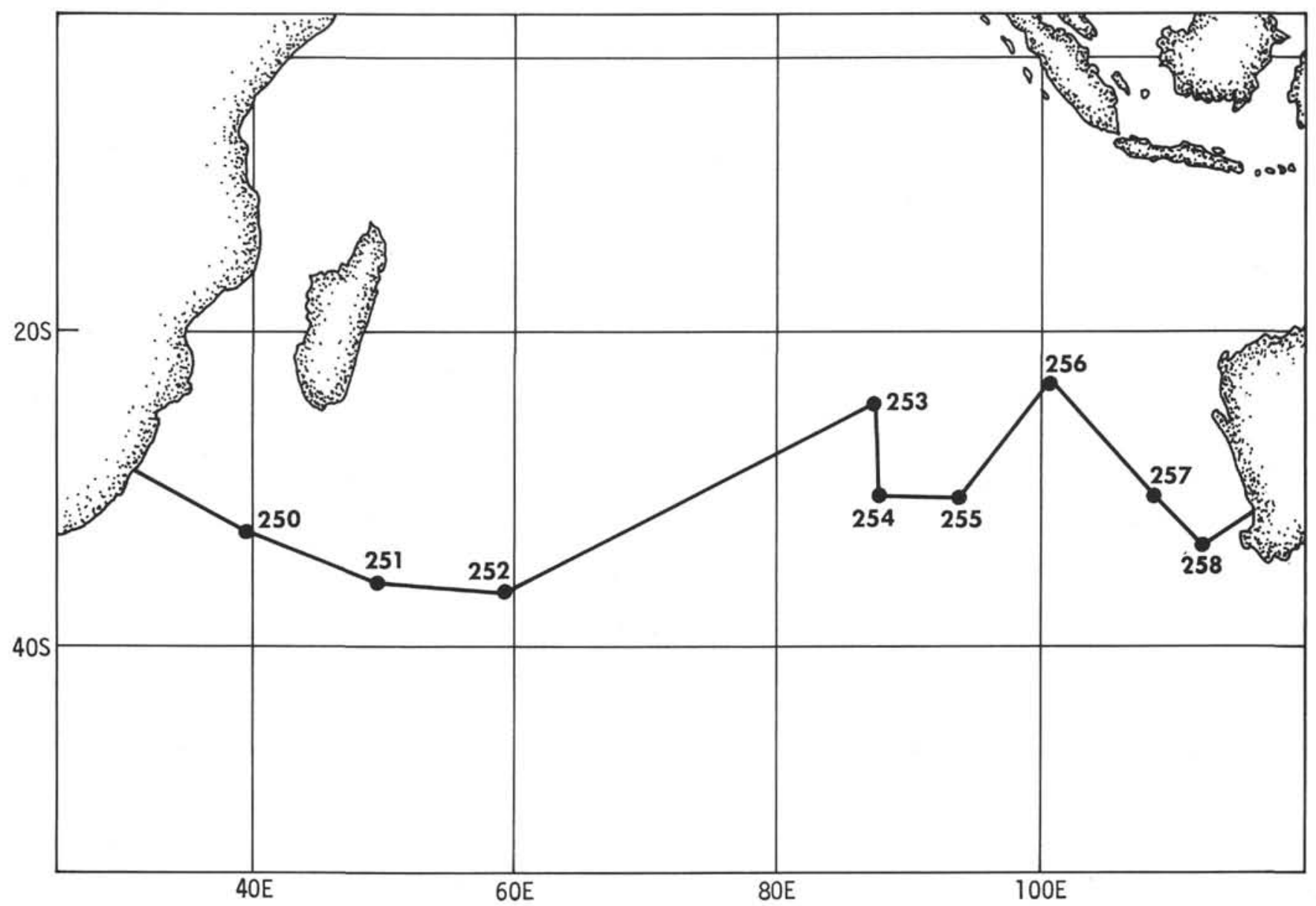

Figure 1. Location of the sites studied.

that in many cases marker species were very scarce, and, for this reason, they were not found in the first 200-250 specimens, but only by checking the remaining residues. In addition, for the location of the Quaternary/Pliocene boundary (see section on Biostratigraphical Boundaries) where the relationship between Globorotalia truncatulinoides and $G$. tosaensis was taken as the main criterion, at least 100 specimens of these species were collected at random from each sample. If the fauna was poor, the whole sample was checked and all the foraminiferal tests found were picked out. In cases where the material was attacked chemically, fewer than 200-250 specimens were found.

The subsequent steps were as follows: taxonomic identification, counting, and mounting of selected specimens in foraminiferal slides.

The following point should be emphasized with respect to taxonomic identification. The confusions which exist in the taxonomy of foraminifera are really enormous (Boltovskoy, 1965). These confusions have serious consequence for all kinds of paleontological conclusions. Therefore special attention should be paid to the identification of the species recorded.

Certainly I do not claim that all the identifications in this paper are correct. However, I do claim that I did everything I possibly could to achieve this goal.
For this purpose, directly after the cruise, I spent several days in Wellington where I compared my identifications with the foraminiferal collections stored in the Geological Survey of New Zealand and discussed many taxonomic problems with micropaleontologists working there. In Buenos Aires all the identifications were compared with a rather rich collection stored in the Museo Argentino de Ciencias Naturales "B. Rivadavia." During my postcruise-meeting trip to the U.S.A. I discussed several species with F. L. Parker, Scripps Institution of Oceanography, La Jolla, California, and R. Todd, U.S. National Museum, Washington. Finally, I spent several days in Washington to compare my fauna with the enormous foraminiferal collections of the Smithsonian Institution. In addition, to give to my colleagues the opportunity to better understand how I interpret the species recorded, I have tried to provide photographs of almost all of the species found (see Plates 1-14).

Counting the recorded specimens of each species permitted me to put on the range charts not only the existence of the species but also their abundance. However, it should be noted that the abundance signs are not precise. Nevertheless, they help to better display the vertical appearance and disappearance of each species. 
Although all the species were determined and counted, not all of them were used for range charts or discussed and figured in the systematical part of this report. Some relatively rare species were excluded. The most important of these are probably those which belong to the Globigerina bulloides group ( $G$. bulloides, $G$. parabulloides, G. praebulloides, G. falconensis, G. ouchitaensis, G. gnaucki, and some others). Their exclusion is justified not only by their limited importance for stratigraphical purposes in the area under study, but also by confusion and uncertainty regarding their taxonomic separation. The very short time between the cruise and the postcruise meeting and subsequent presentation of the finished report for publication is another reason why this group was not studied in detail, and, therefore, not included in the range charts.

All the samples which contained any kind of planktonic foraminiferal contamination were noted on the range charts. They compose somewhat less than 5\% of the total quantity of Neogene samples which were not barren. But what does attract attention is that in twothirds of the contaminated samples the contamination was evidently caused aboard ship, or less probably, during treatment in the laboratory, because Quaternary elements were found mixed with Pliocene or Miocene fauna.

\section{MAJOR BIOSTRATIGRAPHIC BOUNDARIES AND FAUNAL ASSOCIATIONS}

Unfortunately, there is no uniformity of opinion concerning what foraminiferal criteria should be employed to locate the boundaries between different geologic strata. This is explained mainly because different authors have studied material from different areas with distinct climatic conditions, and, thus, with distinct faunas. In addition, correlation is complicated by the disturbance of the geological sequences which can take place in the cores and by the absence (or excessive abundance) of some foraminiferal species due to the effect of selective dissolution. An alternating presence and/or absence of several species in a core may be caused by climatic fluctuations which took place in past geological epochs. Undoubtedly, the absence of uniformity of opinion with respect to boundary location is also explained to a certain degree by the confusion existing in foraminiferal taxonomy.

In locating the major biostratigraphic boundaries in this report, both foraminiferal and nannoplankton data were taken into consideration. In almost all cases a good correlation was observed between the conclusions reached by utilizing each of these two planktonic groups. In several cases, relatively small discrepancies were smoothed over after discussion aboard the Glomar Challenger or during the postcruise meeting and by checking additional samples. In only two cases (Sites 251 and 258) could the differences of opinion with respect to location of the Pliocene/Quaternary boundary not be resolved by mutual discussion. These cases are discussed later.

As for the zonations, different zonal schemes are used by different micropaleontologists. Those best elaborated and based on the richest material are the zonations by Bolli (1966) (the first chart of this zonation from the middle Miocene into the Pliocene was published by Bolli and Bermúdez [1965]) and Blow (1969) (the first chart of this zonation was published by Banner and Blow [1965]).

Although Blow, in his zonation, used letters and numbers to name different zones and this method is unacceptable in the existing stratigraphic code, I find it much more convenient and practical than using specific names. Several species established as marker species by the authors of the zonations cited were not found in the present material. However, by using other species (as well as using the nannoplankton data), it was possible to relate many sequences to the zones established previously. It would seem rather illogical to call a zone by the name of foraminiferal species which was not found either in this zone or in the area. At the same time several species were found which could be considered as marker species for the present area, but they were not mentioned in the zonations of Bolli or Blow. Therefore, I prefer to use Blow's method of calling zones by letters and numbers.

I was unable to find all Blow's zones in the sites studied, and, in many cases, I was forced to distinguish larger units than single zones. This can be explained primarily by the fact that Blow's zonation (as well as Bolli's) is based principally on tropical and subtropical material, whereas sites studied in the present report are located in the temperate zone. An exception is Site 253 which is located in the subtropical zone and where the general character of the assemblages is of warm water. However, detailed zonation at this site was not carried out because, in spite of the subtropical location of the site, several species cited by Blow or Bolli were not found there, and many species recorded in this study are not included in the zonations of Bolli or Blow. In addition, there are discrepancies in the stratigraphic range of several common species; those established by Bolli and Blow are not the same as those established in the Leg 26 material, in general, and at Site 253, in particular.

It should also be mentioned that so-called "systems of datum levels" are in use by some micropaleontologists (Jenkins, 1966; Berggren, 1972). I agree with Beckmann (1971) that these systems can be very practical for initial prompt identification in samples aboard a ship. However, they are not very practical for detailed study in a shore laboratory because dissolution effects can eliminate several marker species. Furthermore, several species can be missing for other reasons or may have somewhat different ranges.

It would be possible to create a new zonation (or a "revised" zonation) on the basis of material collected during Leg 26; however, I do not think that this would be the right way to handle the problem. First of all, I do not think that material of one leg is sufficient to create a new well-founded zonal scheme. Secondly, I am of the opinion that it is much better to try to adjust your results to a well-known zonation than to create a new one based on insufficient evidence. This approach can lead to the possibility of world-wide correlation. Thus, I tried to use Blow's zones wherever possible. 
Several criteria used to locate different boundaries are briefly discussed below. Only the more important and recent published works are cited. By citing specific names, the original forms used by the authors of the papers cited are conserved. The criteria used in this report to locate stratigraphic boundaries, as well as the foraminiferal assemblages typical of different ages, are discussed.

\section{The Oligocene/Miocene Boundary and Subdivision of the Miocene}

Blow (1969) stated that the first appearance of Globigerinoides primordius defines the base of the Miocene, and the extinction of Globigerina angulisuturalis, Globigerina ciperoensis, Turborotalia kugleri, and some other species takes place in the basal Miocene.

According to Bandy and Ingle (1970), the base of the lower Miocene in tropical waters is characterized by the first appearance of Globigerinoides trilobus. Other typical species are: Globigerina concinna, Catapsydrax stainforthi, and Globorotalia suteri. Middle Miocene can be located by the first appearance of "Orbulina" suturalis and upper Miocene by the presence of Globigerina siphonifera, "Orbulina" universa, and Globorotalia menardii

Krasheninnikov (1971) stated that the base of the lower Miocene is marked by the first appearance of Globorotalia kugleri. The base of the middle Miocene is marked by the first appearance of Globorotalia fohsi and G. menardii. G. miocenica's first appearance marks the base of the upper Miocene.

In the present report the following assemblages and paleontological events were considered as indicative for the Oligocene and Miocene periods.

Oligocene marker species: Globigerina ampliapertura, G. angulisuturalis, G. angiporoides, Globorotalia pseudobulloides, and several other species such as Globigerina cryptomphala, G. tripartita, G. yeguaensis, G. euapertura, etc., which extend into the early Miocene.

The Oligocene/Miocene boundary: Globigerina ampliapertura, G. angulisuturalis, and G. angiporoides become extinct. Globoquadrina venezuelana, $G$. altispira, Globigerinoides trilobus, s.l., G. sicanus, Globorotalia miozea conoidea, and Globigerina "praerubra" appear.

Early Miocene: in addition to the above-mentioned species, very typical early Miocene foraminifera are Globigerinita dissimilis dissimilis, $G$. dissimilis ciperoensis, Globorotalia kugleri, and, in the uppermost lower Miocene and lowermost middle Miocene, Globorotalia peripheroronda.

Lower Miocene/middle Miocene boundary: many species which composed the Oligocene fauna become extinct. From this group only Globoquadrina dehiscens dehiscens and Globorotalia opima, s.l. extend into the middle Miocene. Many species, as, for instance, Globigerinoides amplus, G. bulloideus, G. ruber, Globigerinopsis aguasayensis, Globorotalia margaritae, $G$. petaliformis, Orbulina universa, appear.

Middle Miocene: the most typical and/or common foraminifera in the middle Miocene assemblage are Globorotalia miozea conoidea, G. aff. limbata, Globo- quadrina dehiscens dehiscens, G. venezuelana, Globigerina nepenthes, $G$. decoraperta, Globigerinoides bulloideus, $G$. ruber, G. trilobus, G. mitra and Sphaeroidinella seminulina.

Middle Miocene/upper Miocene boundary: appearance of Globorotalia crassaformis, G. limbata, G. menardii, s.l., G. scitula, and Sphaeroidinella subdehiscens.

Upper Miocene: the most typical and/or common species are Globorotalia miozea conoidea, G. limbata, $G$. margaritae, Globigerina nepenthes, $G$. decoraperta, Globigerinoides trilobus, G. bulloideus, G. obliquus, s.l., G. amplus, and Sphaeroidinella seminulina.

\section{The Miocene/Pliocene Boundary and Subdivision of the Pliocene}

This boundary was located by different authors using the following criteria.

Banner and Blow (1965): Sphaeroidinella subdehiscens was replaced by its descendant, $S$. dehiscens.

Bandy and Wade (1967): (a) disappearance of Sphaeroidinella seminulina, Globoquadrina altispira, and Globorotalia tumida miocenica; (b) appearance of Globorotalia inflata and G. truncatulinoides: and (c) great development of Pulleniatina obliquiloculata, Globorotalia puncticulata, and Sphaeroidinella dehiscens.

Jenkins (1967): appearance of Globorotalia inflata, $G$. crassaformis, and G. hirsuta.

Blow (1969): appearance of Globorotalia tumida tumida and the replacement of Sphaeroidinella subdehiscens paenedehiscens by its descendant $S$. dehiscens dehiscens.

Berggren (1969) and Cifelli (1970): appearance of Sphaeroidinella dehiscens.

Bandy et al. (1971): appearance of Sphaeroidinella dehiscens and, very close to this event, the extinction of $S$. subdehiscens and Globoquadrina altispira.

We can summarize by stating that the majority of researchers locate the Miocene/Pliocene boundary where: (a) Sphaeroidinella subdehiscens is replaced by its descendant $S$. dehiscens. (b) Globoquadrina altispira becomes extinct, and (c) Globorotalia inflata and $G$. crassaformis appear.

In the present material Sphaeroidinella dehiscens and $S$. subdehiscens are rare species. Globorotalia inflata and $G$. crassaformis are common and abundant. The latter two species were taken as the main marker species in locating the Pliocene. The frequent and abundant occurrence of these species was used to locate the beginning of the Pliocene. Their first occurrence at almost all sites was in the uppermost Miocene. Globorotalia crassaformis always appeared somewhat earlier than $G$. inflata, and the latter species is probably a better marker species for the beginning of the Pliocene.

In addition, the following species appeared at the beginning of the Pliocene: Globorotalia crotonensis, $G$. hirsuta, and Pulleniatina primalis. The following became extinct: Globigerinita unicava, Globigerinopsis aguasayensis, Globorotalia petaliformis, and Orbulina suturalis. 


\section{The Pliocene}

In the Pliocene sequence Blow (1969) distinguished the upper part of Zone N18, Zones N19 and N20, and the lower part of Zone N21. Parker (1967) considered that entire zones N19, N20, and N21 compose the Pliocene. These three zones can be interpreted as lower, middle, and upper Pliocene, respectively. Parker (1973) gives probably the most clear criteria for the subdivision of the Pliocene in the Atlantic deep-sea section. She uses Sphaeroidinella dehiscens to mark the beginning of the lower Pliocene, Globigerinoides fistulosus, Globorotalia exilis, and $G$. miocenica to mark the beginning of the middle Pliocene, and Globorotalia tosaensis to indicate the beginning of the upper Pliocene. Parker's scheme corresponds rather well to the subdivision recognized in the present study.

The criteria to locate the beginning of the Pliocene were discussed above.

Middle Pliocene was located in the material of the present study where for the first time Globigerinoides fistulosus, G. pyramidalis, Globoquadrina dutertrei, and Globorotalia crassula appeared. Globorotalia exilis was not found at all and $G$. miocenica was determined tentatively. An additional criterion used in the present study to locate the lower/middle Pliocene boundary was the extinction of Globigerina nepenthes and Globigerinoides amplus.

The upper Pliocene was located when Globigerina calida, Globoquadrina humerosa, and Globorotalia truncatulinoides appeared for the first time. Globorotalia tosaensis was recorded for the first time in the middle Pliocene in the present material.

\section{The Pliocene/Quaternary Boundary}

The following criteria have been used by different authors to locate this boundary.

Bandy (1967), Jenkins (1967): change in coiling direction of Globigerina pachyderma from dominantly dextral to sinistral.

Ericson et al. (1964), Ericson and Wollin (1964, 1968): (a) change in coiling direction of Globorotalia menardii from $95 \%$ dextral to $95 \%$ sinistral, (b) disappearance of Globigerinoides sacculifer fistulosa, (c) extinction of Globorotalia multicamerata, (d) increase in side of Globorotalia menardii, (e) appearance in abundance of Globorotalia truncatulinoides. The latter criterion is the most important according to the authors cited (Ericson and Wollin, 1968).

Parker (1967): disappearance of Globigerinoides fistulosus and Globoquadrina pseudofoliata, appearance of Globigerinoides tenellus.

Berggren et al. (1967), Berggren (1969), Bandy and Wilcoxon (1970), Bolli (1970), Cifelli (1970), Hays and Berggren (1971), and Poag (1971): appearance of Globorotalia truncatulinoides.

Blow (1969): appearance of Globorotalia tosaensis tenuitheca from its ancestor, $G$. crassaformis oceanica.

Lamb (1969): extinction of Globoquadrina altispira and appearance in the lower Pleistocene of Globorotalia truncatulinoides.
The replacement of Globorotalia tosaensis by $G$. truncatulinoides is the most universally accepted of the criteria listed above. Unfortunately, no unity of opinion exists as to the exact location of the Pliocene/Quaternary boundary using this criterion. Should this boundary be placed (a) at the last appearance of Globorotalia tosaensis? (b) at the first appearance of Globorotalia truncatulinoides? (c) when Globorotalia truncatulinoides appears in appreciable quantity? (d) when only Globorotalia truncatulinoides is found and no specimens of $G$. tosaensis? The vertical distribution of the Globorotalia tosaensis/G. truncatulinoides group observed at Site 258 illustrates well that the location of the boundary depends very much on the criterion accepted. All the specimens of these two species found at the site mentioned were counted. Their vertical distribution can be described as follows.

The first geological appearance of Globorotalia tosaensis (isolated specimens) at this site was recorded at a depth of 55.5 meters (the uppermost middle Pliocene). At a depth of 45.5 meters the first specimens of Globorotalia truncatulinoides were found (upper Pliocene). In the layer between 45.5 and 30.20 meters both species were present, but Globorotalia tosaensis was almost always more numerous than $G$. tosaensis. Between 30.20 and 28 meters both species were found in approximately the same quantity. Starting from 28 meters upward, Globorotalia truncatulinoides became more numerous than $G$. tosaensis. At a depth of approximately 19 meters Globorotalia truncatulinoides became abundant. At a depth of 2.20 meters the last specimen of Globorotalia tosaensis was found. The layer between 2.20 and 0 meters contained only Globorotalia truncatulinoides.

Now, where should the Pliocene/Quaternary be located? At a depth of 45.5 meters? At a depth of 19 meters? Or at a depth of 2.20 meters?

Another rather good example is seen at Site 251. The appearance and disappearance of Globorotalia truncatulinoides and $G$. tosaensis observed at this site is as follows: First appearance of Globorotalia tosaensis was at a depth of 59 meters. First appearance of Globorotalia truncatulinoides was at a depth of 54.5 meters. $G$. truncatulinoides was more numerous than $G$. tosaensis at 37 meters, and $G$. truncatulinoides was abundant at 30 meters. The uppermost appearance of $G$. tosaensis is at 5 meters.

The distributions described are a natural consequence of the fact that the replacement of Globorotalia by $G$. truncatulinoides was a rather long process which took considerable time and was accompanied by the formation of a series of intermediate forms.

In the present study the following criterion was taken to separate the Quaternary from the Pliocene by means of Globorotalia truncatulinoides $/ G$. tosaensis data. As mentioned above, in addition to $200-250$ planktonic specimens picked out at random from each sample, at least 100 specimens of Globorotalia truncatulinoides $/ G$. tosaensis group were also separated at random. The relationship between the species was calculated for every sample. If this ratio was more than 1 (meaning that 
Globorotalia truncatulinoides predominated over $G$. tosaensis), the sample was considered Quaternary. If this ratio was less than 1 , the core was considered to be Pliocene.

This criterion was taken as the principal one to locate the Pliocene/Quaternary boundary at all sites. At four sites $(250,253,254$, and 255$)$ the boundary located in this way corresponded exactly with the boundary determined using nannoplankton. At two sites (250 and 258) foraminiferal method gave a location for the boundary of 10-15 meters higher than the nannoplankton method. As both sites are located quite far south, perhaps climatic conditions (lower temperatures) are the reason for this discrepancy. Opinions have been expressed (Kennett and Geitzenauer [1969]), that Globorotalia truncatulinoides and G. tosaensis are phenotypic variants or separate taxa with distinct environmental preferences. The latter species preferred colder water and in colder water it was more numerous. Thus, at Sites 250 and 258, the coldest, Globorotalia tosaensis was more numerous and, for this reason, the Pliocene/Quaternary boundary is higher than that located by means of nannoplankton data. However, it should be pointed out that the location of the boundary in question at Sites 251 and 258 was checked using other foraminiferal faunal criteria. In summary, it is not clear why there is a discrepancy in the placement of the Pliocene/Quaternary boundary at these sites.

\section{The Quaternary}

The Quaternary lasted about 1.8 m.y. (Berggren, 1972). It is divided into Pleistocene and Holocene. Different points of view exist regarding the number, character, and duration of glacial epochs which took place during the Pleistocene. Material collected for the usual work carried out with Glomar Challenger material is not suitable for study of Quaternary paleoclimatic fluctuations because this kind of study requires closely spaced samples. However, to carry out a study of the above-mentioned fluctuations at Site 253, a series of samples at $10-\mathrm{cm}$ intervals was taken. The results are published in this volume as a separate paper entitled "Late Pliocene and Quaternary paleoclimatic changes."

The Holocene is postglacial time, and its duration, according to the majority of researchers, has been 11,000 years. The Pleistocene/Holocene boundary is rather difficult to locate faunally, because the faunas are practically identical. There are differences, but they are very insignificant and do not have world-wide application.

Blow (1969), for instance, interpreted his Zone N23 as the Holocene and probably the uppermost part of the Pleistocene. He emphasized that the appearance of two new species are recorded, namely Hastigerina adamsi and Hastigerinella digitata. In addition, Pulleniatina obliquiloculata finalis and Sphaeroidinella dehiscens excavata became abundant.

Parker (1973) separates the Holocene from the Pleistocene using the presences of pink-walled Globigerina rubescens and Globigerinoides ruber in the Pleistocene. Boltovskoy (1968) separated pink-walled specimens of the latter species under the name Globigerinoides ruber, forma rosea, to distinguish them from white-walled specimens, $G$. ruber, forma alba. The criterion of Parker perhaps is not very good. Orr (1969) and Jenkins and Orr (1972) considered that the preservation of pigmented specimens depends on the sedimentation rate. Rapid sedimentation rates allowed for the preservation of more pigmented specimens than did low rates.

For the temperate zone a good criterion is the general change of fauna to the more warm water type. As Globorotalia menardii is a typical warm-water species, Ericson et al. (1964) and Ericson and Wollin (1968) used its appearance in abundance to determine the end of the Pleistocene.

Bandy (1967) used the change in coiling direction of Globigerina pachyderma from sinistral to dextral.

In only two sites on Leg 26 was it possible to separate Holocene from Pleistocene deposits. At Site 258 the Holocene/Pleistocene boundary was located using Parker's criterion. The results were rather unexpected; the Holocene sediments appeared to be 8.5 meters thick. At Site 253 the boundary was located by means of a detailed paleoclimatic study utilizing the relationship between Globorotalia menardii and $G$. inflata (Boltovskoy, Late Pliocene and Quaternary paleoclimatic changes, this volume). The results obtained were much more as expected, namely the limit between Holocene and Pleistocene was fixed approximately at a depth of $30 \mathrm{~cm}$ from the bottom surface.

\section{DESCRIPTION OF THE SITES}

The range charts of selected species (Figures 2-7) show the most important changes which occurred in foraminiferal fauna from early Miocene to Recent in the middle part of the Indian Ocean. Qualitative, as well as quantitative, data are noted on these charts. As was explained above, some species were not studied in detail for several reasons, and, thus, are not included in the range charts. An examination of the range charts also gives an idea of what criteria were used by the author in locating the biostratigraphic boundaries at each site. The problem of biostratigraphical boundaries was previously discussed and is also treated in the following descriptions of the sites.

\section{Site 250 (Figure 2)}

Site 250 is located in the Mozambique Basin. Two holes, 250 and $250 \mathrm{~A}$, were cored. Hole 250 was cored from the surface to a depth of 18.5 meters, then drilled to a depth of 55.5 meters and then cored again from 55.5 to 65 meters. Hole $250 \mathrm{~A}$ was continuously cored from 54.5 to 83 meters and then discontinuously cored from 115.5 to 738.5 meters. The Neogene sequence encountered at this site is 672 meters thick and consists of clayey coccolith ooze, detrital silty clays, and detrital clay.

The foraminiferal fauna from this sequence has been affected by solution and, in many sections, has been completely dissolved. Assemblages found are poor 
qualitatively and quantitatively and not very suitable for use either in age determination or for drawing ecological conclusions.

At least the uppermost 83 meters are Quaternary in age. No way was found to separate Holocene from Pleistocene sediments. The foraminiferal assemblage recorded from the Quaternary deposits is evidently of a temperate zone type. Globorotalia inflata, s.l., which is a typical cold-temperate species, strongly predominates. It is accompanied by: (a) cold-temperate species such as Globigerina pachyderma (dex.) and G. quinqueloba, Globorotalia truncatulinoides/tosaensis; (b) warmtemperate species, Globorotalia hirsuta, G. cf. humilis, and Globigerinella aequilateralis; and (c) a few warmwater species, Globorotalia menardii, Globigerinoides ruber, $G$. conglobatus, and $G$. elongatus. The whole assemblage indicates lower temperature than observed in the same area today. Benthonic species indicative of great depths were found (Cibicides kullenbergi, Epistominella japonica, and Nonion soldanii), and many typical deep-water Fissurina, Parafissurina, Lagena, etc.). The assemblage does not show any important changes through the Quaternary.

Cored material found below the Quaternary deposits is late Pliocene in age. It contains more or less the same poor fauna but without Globorotalia truncatulinoides. Globorotalia tosaensis, was found, however. The thickness of the late Pliocene sediments is at least 43.5 meters. It is very probably more because some parts of the section were not cored, and a part of the cored section appeared to be barren.

Neither middle nor lower Pliocene deposits were recorded.

At the depth of 235 meters the upper Miocene (Zones N16-N18) was found. The fauna is still poorer qualitatively and quantitatively than the Pliocene fauna. The absence of several typical Pliocene species (Globorotalia inflata, s.l.; G. crotonensis; Sphaeroidinella dehiscens; Pulleniatina obliquiloculata, s.s., etc.) indicates a Miocene age for the sediments.

A great sequence of completely barren sediments (about $300 \mathrm{~m}$ ) was drilled below the upper Miocene section. Only one sample in this section contained debris of some arenaceous benthonic foraminifera.

Below this sequence about 40 meters of lower Miocene were encountered. The sediments were partially barren, but, in some places, contained a rather typical lower Miocene fauna (Globorotalia kugleri, G. peripheroronda, Globigerinita dissimilis dissimilis, etc.). They can be considered as Zones N4-N8. In addition, some evidently reworked Eocene and Oligocene foraminifera were recorded from these lower Miocene sediments. Some arenaceous benthonic foraminifera of long-ranging species were found, too.

An assemblage of calcareous benthonic foraminifera found in Sample 18, CC is of special interest and rather enigmatic. This assemblage consisted of specimens of Rotalia cubensis, Reussella spinulosa, Bolivina tortuosa, Elphidium crispum, Nodobaculariella cassis, Sphaeroidina bulloides, Quinqueloculina bradyana, and some other shallow-water species typical of a subtropical and even a tropical zone. All the specimens were in a very good state of preservation and belong to the Recent fauna, although it is possible that they also lived during the Miocene. If this assemblage contained also Recent planktonic foraminifera, I would think it to be the result of contamination aboard Glomar Challenger. However, no typical specimens of Recent planktonic species were found in the sample. Thus, it is more logical to assume that the benthonic assemblage mentioned is the result of sediment transport from shallower areas which occurred during the early Miocene.

A very few damaged benthonic calcareous specimens were recorded in Sample 17, CC; however, no typical shallow water dwellers were found among them.

\section{Site 251 (Figure 3)}

This site is located about $180 \mathrm{~km}$ north of the middle part of the Southwest Branch of the Indian Ridge. Two holes were drilled. Hole 251 extended from 0 to 87.5 meters, subbottom; it was stopped for technical reasons. The whole sequence was cored. Hole 251A was drilled to a depth of 489 meters in sediments and an additional 10 meters into the underlying basalt and cored intermittently (276.5 m were cored, $158.4 \mathrm{~m}$ were recovered). The Neogene-Recent sequence consisted of 486.5 meters of nannoplankton ooze and chalk.

Site 251 is paleontologically very interesting and valuable because it has yielded a complete, and presumably, uninterrupted, midlatitudinal Neogene sequence for the Indian Ocean. Unfortunately, however, owing to the great depth, the fauna was affected by solution, insignificantly in Quaternary deposits, but considerably in the Miocene, especially the lower Miocene. In several Miocene samples the foraminiferal assemblages consisted of only two to four of the most resistant species. There were samples in lower Miocene which contained only very small-sized, practically unidentifiable specimens. These factors considerably decreased the value of the site as a possible standard.

In all the assemblages down to the middle and lower Pliocene Globorotalia inflata, s.l. predominates. It is always accompanied by Globorotalia crassaformis which in the Pliocene sediments is not as numerous as the former species. Only in the upper Miocene does Globorotalia crassaformis become more abundant than $G$. inflata. In a part of the upper and middle Miocene section the predominant species is Globorotalia miozea conoidea. Globigerina pachyderma (dex.), G. bulloides, Globorotalia truncatulinoides/tosaensis, Globigerinella aequilateralis, Orbulina universa, Globigerinoides ruber, and $G$. trilobus, s.l. are other species which compose the Quaternary and Pliocene assemblages. The whole fauna is typical of the temperate zone. Such a characteristic warm water species as Globorotalia menardii, s.l. is found sporadically and as isolated specimens.

The changes in the relationship between warm-water and cold-water faunas suggest some climatic fluctuations. However, these fluctuations were relatively insignificant and not well pronounced.

The thickness of the Quaternary deposits is $\mathbf{3 7}$ meters. It was not possible to divide them into Holocene and Pleistocene. 


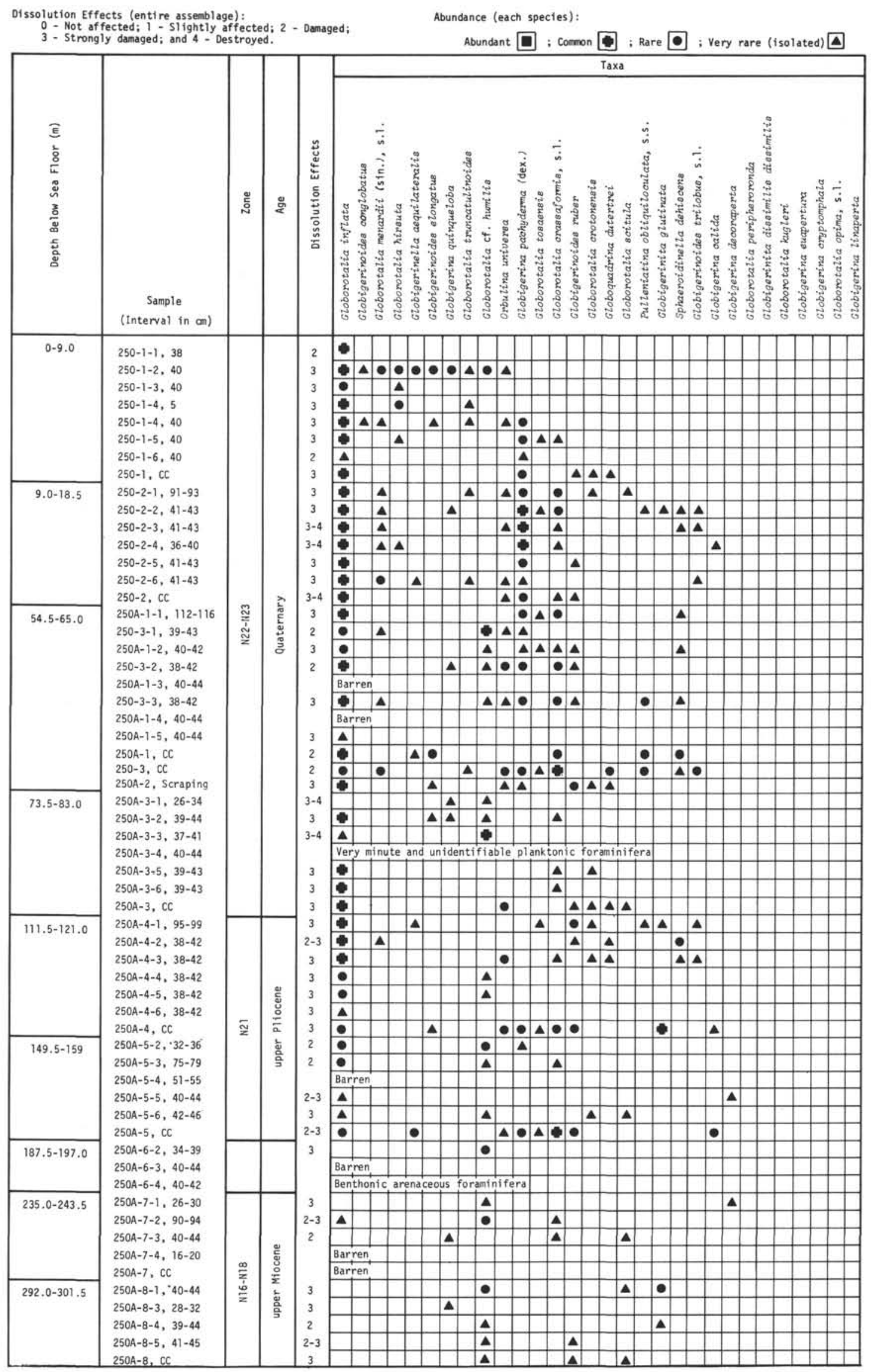

Figure 2. Range chart of selected Planktonic Foraminifera species at Site 250 (Holes 250 and 250A). 
Dissolution Effects (entire assemblage):

- Not affected, Slightly aefected; 2 - Damaged

Abundance (each species):

Abundant $\square$; Common $\square$; Rare $\square$; Very rare (isolated) $\boldsymbol{\Delta}$

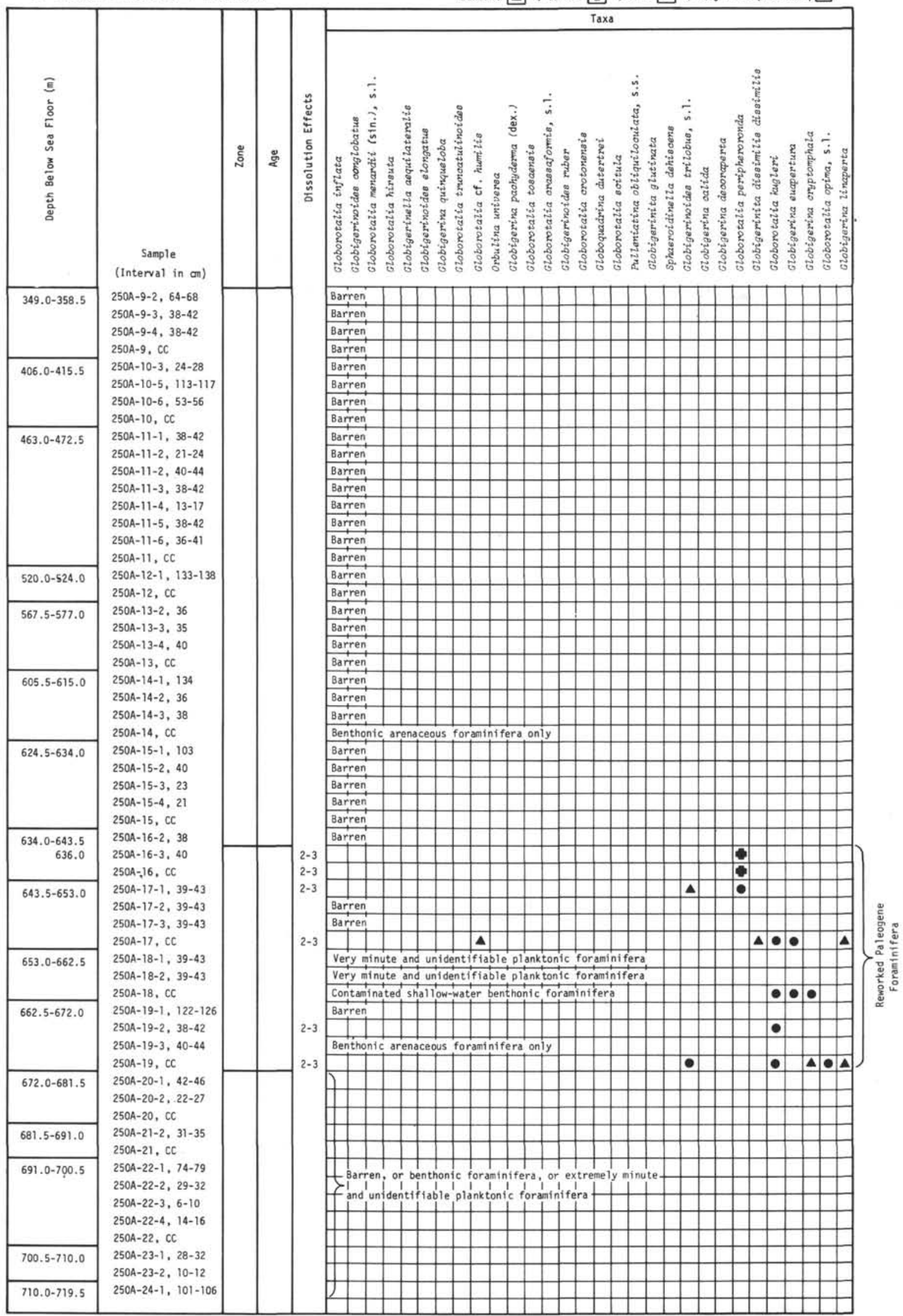

Figure 2. (Continued). 


\begin{tabular}{|c|c|c|c|c|c|c|c|c|c|c|c|c|c|c|c|c|c|c|c|c|c|c|c|c|c|c|c|c|c|c|c|c|c|}
\hline & & & & & & & & & & & & & & & & & & & & & Ta: & & & & & & & & & & & & \\
\hline 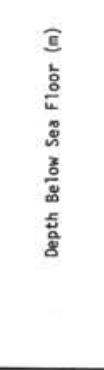 & $\begin{array}{c}\text { Sample } \\
\text { (Interval in on) }\end{array}$ & 气̆ & \& & 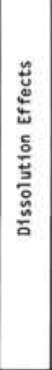 & 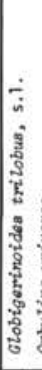 & : & 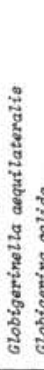 & 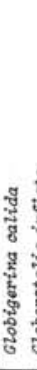 & 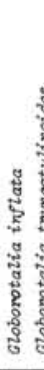 & 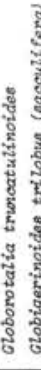 & 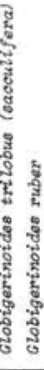 & 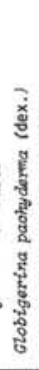 & 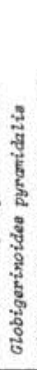 & 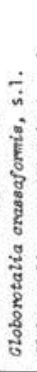 & 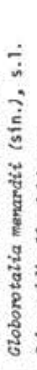 & & & 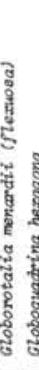 & 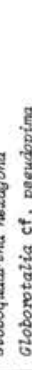 & & 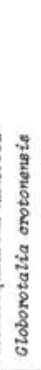 & 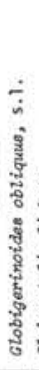 & & 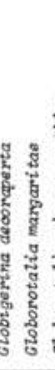 & 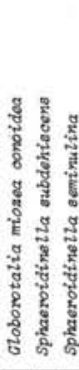 & 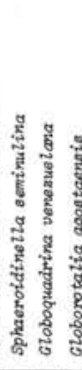 & & 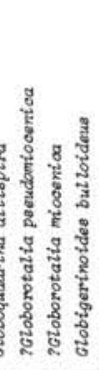 & 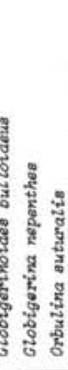 & 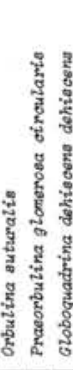 & 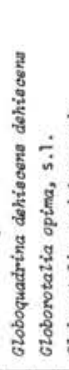 & 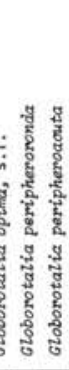 & \\
\hline $0-2.0$ & $251-1-1,96-100$ & & & 0 & \begin{tabular}{|l|l}
$\Delta$ & \\
\end{tabular} & e. & - & $\mathbf{A}$ & ec & - & & & & & & & & & & & & & & & & & & 1 & & & & & \\
\hline & $251-1-2,20-24$ & & & 0 & & $\bullet$ & - & $\mathbf{A}$ & 6 & - 4 & \begin{tabular}{l|l}
$\mathbf{A}$ & $\mathbf{\Delta}$ \\
\end{tabular} & - & & 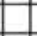 & & & & & & & & & & & & & & & & & & & \\
\hline & $251-1, \mathrm{CC}$ & & & 0 & $\bullet$ & A & - & $\Delta$ & 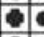 & $\bullet$ & $\Delta$ & $\Delta$ & $\mathbf{A}$ & $\Delta$ & & & & & & & & & & & & & & & & & & & \\
\hline $2.0-11.5$ & $251-2-1,39-41$ & & & 1 & \begin{tabular}{|l|l} 
& 4 \\
\end{tabular} & $\Delta$ & - & & 96 & - & L & 1 & & & & & & & & & & & & & & & & & & & & & \\
\hline & $251-2-2, \quad 39-41$ & & & 1 & $\Delta$ & 6 & - & - & 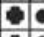 & - & $\Delta$ & 1 & & $\Delta$ & & & & & & & & & & & & & & & & & & & \\
\hline & $251-2-3,40-42$ & & & 1 & $\mathbf{A}$ & - & & & 6 & - & $\Delta$ & 6 & & & $\operatorname{sign}$ & nifi & can & $m$ & ixtu & & of $\mathrm{re}$ & & rked & Mioce & ene speci & cimens & & & & & & & \\
\hline & $251-2-4,40-42$ & & & 1 & & $\mathbf{\Delta}$ & $\Delta$ & & 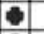 & & $\mathbf{A}$ & $\bullet$ & & & $\Delta$ & & & & & & & & & & & & & & & & & & \\
\hline & $251-2-5,40-42$ & & & 1 & & $\Delta$ & $\Delta$ & & 8 & $\Delta \mid$ & $\Delta \cdot$ & - & & $\mathbf{A}$ & $\mathbf{A}$ & $\mathbf{\Delta}$ & $\mathbf{\Delta}$ & & & & & & & & & & & & & & & & \\
\hline & $251-2-6,40-42$ & & & 1 & & $\Delta$ & - & & 6 & $\Delta 4$ & \begin{tabular}{|l|l}
$\mathbf{A}$ & $\mathbf{\Delta}$ \\
\end{tabular} & - & & $\mathbf{A}$ & $\mathbf{A}$ & & $\Delta$ & & & & & & & & & & & & & & & & \\
\hline & $251-2, \mathrm{CC}$ & & & $1-2$ & & $\Delta$ & $\Delta$ & & - & & $\bullet$ & - & & $\mathbf{A}$ & & $\Delta$ & & & & & & & & & & & & & & & & & \\
\hline $11.5-21.0$ & $251-3-2,40-42$ & $\tilde{\mathbb{N}}$ & 챝 & 2 & \begin{tabular}{|l|l}
$\mathbf{\Delta}$ \\
\end{tabular} & $\Delta$ & $\bullet$ & & 6 & $\Delta$ & $\Delta$ & $\bullet$ & & $\Delta$ & & & & & & & & & & & & & & & & & & & \\
\hline & $251-3-3,40-42$ & ָัָ & E & 2 & & $\Delta$ & & & 64 & $\Delta \mathbf{A}$ & \begin{tabular}{|l|l}
$\mathbf{\Delta}$ & $\mathbf{\Delta}$ \\
\end{tabular} & $\Delta$ & & - & $\Delta$ & & & & & & & & & & & & & & & & & & \\
\hline & $251-3, \mathrm{CC}$ & $\stackrel{\mathbb{N}}{z}$ & 范 & 2 & & $\bullet$ & - & & 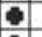 & & $\Delta$ & $\Delta$ & & $\bullet$ & $\Delta$ & & & & & & & & & & & & & & & & & & \\
\hline $21.0-30.5$ & $251-4-1,40-42$ & & & 2 & & - 1 & $\Delta$ & & 단 & & & $\mathbf{\Delta}$ & & - & & & & & & & & & & & & & & & & & & & \\
\hline & $251-4-2,40-42$ & & & 2 & & $\Delta$ & $\mathbf{\Delta}$ & $\Delta$ & 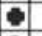 & & & $\bullet$ & $\Delta$ & $\Delta$ & $\Delta$ & & & & & & & & & & & & & & & & & & \\
\hline & $251-4-3,40-42$ & & & 2 & & $\Delta$ & & & - & & & $\bullet$ & & $\bullet$ & $\Delta$ & & & & & & & & & & & & & & & & & & \\
\hline & $251-4-4,40-42$ & & & 2 & & $\Delta$ & - & & 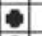 & & & & & - & & & & & & & & & & & & & & & & & & & \\
\hline & $251-4-5,40-42$ & & & 2 & & $\bullet$ & $\mathbf{A}$ & $\mathbf{A}$ & $\theta$ & & 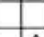 & 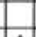 & & - & $\mathbf{A}$ & & & & & & & & & & & & & & & & & & \\
\hline & $251-4-6,40-42$ & & & 2 & & \begin{tabular}{|c|c}
$\mathbf{A}$ \\
\end{tabular} & - & & 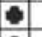 & & $\Delta$ & $\Delta$ & & $\bullet$ & & & & & & & & & & & & & & & & & & & \\
\hline & $251-4, \mathrm{CC}$ & & & 2 & & $\Delta$ & $\Delta$ & & - & & & $\mathbf{A}$ & & $\bullet$ & & & & & & & & & & & & & & & & & & & \\
\hline $30.5-40.0$ & $251-5-2,40-42$ & & & 1 & & $\mathbf{A}$ & $\Delta$ & & - & & & & & $\Delta$ & $\Delta$ & & & & & & & & & & & & & & & & & & \\
\hline & $251-5-4,40-42$ & & & 1 & & $\Delta$ & $\Delta$ & & 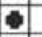 & & $\Delta$ & & & - & & & & & & & & & & & & & & & & & & & \\
\hline & $251-5-5,40-42$ & & & 1 & & $\Delta$ & - & & 6 & & 1 & & & - & $\Delta$ & & & & & & & & & & & & & & & & & & \\
\hline & $251-5-6,40-42$ & & & 1 & \begin{tabular}{|l|l}
$\Delta$ \\
\end{tabular} & $\bullet$ & $\Delta$ & & 당 & & $\mathbf{\Delta} \mathbf{\Delta}$ & & & $\bullet$ & $\Delta$ & & $\mathbf{\Delta}$ & & & & & & & & & & & & & & & & \\
\hline & $251-5, \mathrm{CC}$ & & & 1 & \begin{tabular}{|l|l}
$\mathbf{A}$ \\
\end{tabular} & & - & & 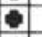 & & $\mathbf{\Delta}$ & & & - & & $\mathbf{A}$ & - & $\mathbf{A}$ & 4 & $\Delta$ & & & & & & & & & & & & & \\
\hline $40.0-49.5$ & $251-6-1,40-42$ & & & 2 & & $\bullet$ & - & & 6 & & $\Delta$ & & & - & $\Delta$ & & & & 6 & & & & & & & & & & & & & & \\
\hline & $251-6-2,40-42$ & & & 2 & & $\bullet$ & & & 붕 & & $\Delta$ & & & & $\Delta$ & $\Delta$ & & & & & & & & & & & & & & & & & \\
\hline & $251-6-3,40-42$ & & & 2 & \begin{tabular}{|l|l|}
$\Delta$ \\
\end{tabular} & $\bullet$ & $\Delta$ & & 붕 & & \begin{tabular}{l|l}
$\Delta$ & $\Delta$ \\
\end{tabular} & & & $\bullet$ & & $\rightarrow$ & & & & & & & & & & & & & & & & & \\
\hline & $251-6-4,40-42$ & & & 2 & & $\bullet$ & $\bullet$ & & $\bullet$ & & & & & $\bullet$ & & $\Delta$ & & & & $\Delta$ & & & & & & & & & & & & & \\
\hline & $251-6-5,40-42$ & & & 2 & & - & $\bullet$ & & 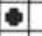 & & $\bullet$ & & & $\bullet$ & & $\Delta$ & & & & & & & & & & & & & & & & & \\
\hline & $251-6-6,40-42$ & & & 2 & & $\Delta$ & & & $\bullet$ & & \begin{tabular}{l|l}
$\mathbf{\Delta}$ \\
\end{tabular} & & & & & $\Delta$ & & & & & & & & & & & & & & & & & \\
\hline & $251-6, \mathrm{CC}$ & & & 2 & & $\bullet$ & $\Delta$ & $\mathbf{A}$ & 붕 & 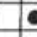 & - & & & - & $\Delta$ & $\Delta$ & - & & & & 0 & $\mathbf{A}$ & & & & & & & & & & & \\
\hline $49.5-59.0$ & $251-7-1,40-42$ & & & 2 & & $\Delta$ & & & 당 & & $\bullet$ & & & $\Delta$ & $\mathbf{A}$ & & 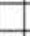 & & & & $\bullet$ & & & & & & & & & & & & \\
\hline & $251-7-2,40-42$ & & & 2 & $\bullet$ & $\mathbf{A}$ & & & $\theta$ & & $\Delta$ & & & $\Delta$ & & $\mathbf{A}$ & $\Delta$ & & & & $\Delta$ & & & & & & & & & & & & \\
\hline & $251-7-3,40-42$ & & & 2 & & $\Delta$ & & & 5 & & $\bullet$ & & & 1 & & t & & & & & $\mathbf{A}$ & & & & & & & & & & & & \\
\hline & $251-7-4,40-42$ & & & 2 & \begin{tabular}{|l|l|}
$\Delta$ \\
\end{tabular} & $\mathbf{A}$ & & & 6 & & $\Delta \bullet$ & - & & $\Delta$ & $\bullet$ & $\mathbf{A}$ & & & & & $\Delta$ & & & & & & & & & & & & \\
\hline & $251-7-5,40-42$ & & & 2 & $\Delta$ & $\Delta$ & & & 둥 & 4 & $\mathbf{A} \mathbf{A}$ & & & $\mathbf{A}$ & $\mathbf{\Delta}$ & $\Delta$ & & & & $\Delta$ & $\Delta$ & & & & & & & & & & & & \\
\hline & $251-7-6,30-32$ & & & 2 & \begin{tabular}{|l|l}
$\mathbf{\Delta}$ \\
\end{tabular} & $\Delta$ & $\Delta$ & & • & & $\Delta \mathbf{\Delta}$ & & & 1 & & $\Delta$ & & & & 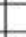 & $\bullet$ & & & & & & & & & & & & \\
\hline & $251-7, \mathrm{CC}$ & & & 2 & $\Delta$ & - & - & $\Delta$ & 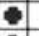 & & & & $\bullet$ & 한 & $\bullet$ & $\Delta$ & $\Delta$ & & & $\bullet$ & $\Delta$ & & & & & & & & & & & & \\
\hline $59.0-68.5$ & $251-8-1,40-42$ & & & $1-2$ & \begin{tabular}{|l|l}
$\Delta$ \\
\end{tabular} & $\bullet$ & $\Delta$ & & 후 & & $\Delta$ & & & - & & & $\Delta$ & & & $\bullet$ & $\Delta$ & & & & & & & & & & & & \\
\hline & $251-8-2,40-42$ & & & $1-2$ & \begin{tabular}{l|l}
$\mathbf{A}$ \\
\end{tabular} & $\Delta$ & & & 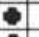 & & $\Delta$ & & & $\Delta$ & & $\Delta$ & & & 4 & & & & & & & & & & & & & & \\
\hline & $251-8-3,40-42$ & & & $1-2$ & \begin{tabular}{l|l}
$\mathbf{A}$ \\
\end{tabular} & $\Delta$ & & & 둘 & & & & & $\Delta$ & & t & & & 4 & & $\Delta$ & & & & & & & & & & & & \\
\hline & $251-8-4,40-42$ & & & $1-2$ & & $\bullet$ & $\Delta$ & $\Delta$ & - & 4 & $\mathbf{\Delta}$ & & & - & & $\mathbf{\Delta}$ & $\Delta$ & & & $\Delta$ & $\Delta$ & $\mathbf{A}$ & & & & & & & & & & & \\
\hline & $251-8-5,40-42$ & & & $1-2$ & $\mathbf{A}$ & $\Delta$ & $\Delta$ & & 6 & & $\Delta$ & & & & & $\mathbf{A}$ & & & & & $\bullet$ & & & & & & & & & & & & \\
\hline & $251-8-6,40-42$ & & हूँ & $1-2$ & & - & $\bullet$ & & 5 & & $\Delta$ & & $\Delta$ & $\bullet$ & & & & & & & $\mathbf{A}$ & & $\Delta$ & & & & & & & & & & \\
\hline & $251-8, \mathrm{CC}$ & & $\stackrel{\circ}{\circ}$ & $1-2$ & $\Delta$ & $\bullet$ & & & 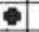 & & - & & & - & & & & & 4 & $\Delta$ & $\bullet$ & & & & & & & & & & & & \\
\hline $78.0-87.5$ & $251 \mathrm{~A}-1-1,40-42$ & 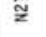 & $\overline{2}$ & 2 & & - & & & - & 4 & $\Delta$ & & & - & & $\Delta$ & & & & & $\Delta$ & & & & & & & & & & & & \\
\hline & $251-10-2,40-42$ & & ฏ & 2 & $\bullet$ & $\Delta$ & $\Delta$ & & - & & $\bullet$ & $\Delta$ & & $\bullet$ & & & & & & & $\Delta$ & & $\Delta$ & & & & & & & & & & \\
\hline & $251 A-1-2,40-42$ & & & 2 & $\bullet$ & \begin{tabular}{|l|l}
$\mathbf{A}$ & -1 \\
\end{tabular} & $\mathbf{\Delta}$ & & $\theta$ & & - & $\Delta$ & & - & & & & & & & $\Delta$ & & $\mathbf{A}$ & & & & & & & & & & \\
\hline & $251-10-3,40-42$ & & & 2 & $\Delta$ & $\bullet$ & $\Delta$ & & 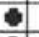 & & $\Delta$ & & & $\bullet$ & & & $\Delta$ & & 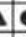 & & $\bullet$ & & & & & & & & & & & & \\
\hline & $251 \mathrm{~A}-1-3,40-42$ & & & 2 & $\Delta$ & $\bullet$ & $\Delta$ & & 한 & & $\Delta$ & & & $\bullet$ & & & $\mathbf{\Delta}$ & 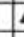 & 1 & & $\bullet$ & & & & & & & & & & & & \\
\hline & $251-10-4,40-42$ & & & 2 & $\Delta$ & $\bullet$ & & & 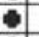 & & $\Delta$ & $\bullet$ & & $\bullet$ & & & & & 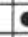 & & $\bullet$ & & & & & & & & & & & & \\
\hline & $251 A-1-4,40-42$ & & & 2 & $\Delta$ & $\bullet$ & & & 9 & & $\Delta$ & - & & - & & & & & 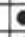 & & $\bullet$ & & & & & & & & & & & & \\
\hline & $251-10-5,40-42$ & & & 2 & $\Delta$ & A & & & 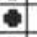 & & $\Delta$ & - & & $\bullet$ & & $\Delta$ & & & 4 & & - & & & & & & & & & & & & \\
\hline & $251 A-1-5,40-42$ & & & 2 & $\mathbf{A}$ & $\Delta$ & & & 9 & & $\Delta$ & - & & $\bullet$ & & $\Delta$ & & & 4 & & - & & & & & & & & & & & & \\
\hline & $251 \mathrm{~A}-1-6,40-42$ & & & 2 & $\Delta$ & $\Delta$ & & & 당 & & & $\bullet$ & & - & & & & & 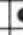 & & - & & & & & & & & & & & & \\
\hline & $251-10, \mathrm{CC}$ & & & $2-3$ & \begin{tabular}{|l|l}
$\Delta$ \\
\end{tabular} & $\bullet$ & $\Delta$ & & $\bullet$ & 4 & $\Delta$ & & & - & & $\mathbf{A}$ & & & & & $\bullet$ & & & & & & & & & & & & \\
\hline & $251 \mathrm{~A}-1, \mathrm{CC}$ & & & $2-3$ & \begin{tabular}{|l|l|}
$\Delta$ \\
\end{tabular} & - & $\Delta$ & & e & 4 & $\mathbf{A} \mathbf{A}$ & & & 방 & & $\Delta$ & & & & & 바 & & & & & & & & & & & & \\
\hline $87.5-95.5$ & $251 \mathrm{~A}-2-1,40-42$ & & & $2-3$ & & $\Delta$ & & & $\bullet$ & & & & & & & & & & 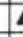 & & $\bullet$ & & & & & & & & & & & & \\
\hline & $251 \mathrm{~A}-2-3,40-42$ & & & $2-3$ & \begin{tabular}{|l|}
$\mathbf{\Delta}$ \\
\end{tabular} & $\Delta$ & $\Delta$ & & 불 & & $\Delta$ & - & & - & & & & & & & & & $\Delta$ & & & & & & & & & & \\
\hline & $251 A-2-4,40-42$ & & & $2-3$ & & $\mathbf{\Delta}$ & $\Delta$ & & - & & & $\Delta$ & & $\bullet$ & & & & & & & $\bullet$ & & & & & & & & & & & & \\
\hline & $251 A-2-5,40-42$ & & & $2-3$ & & & & & 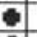 & & 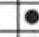 & $\Delta$ & & - & & & & & & & - & & & & & & & & & & & & \\
\hline & $251 \mathrm{~A}-2, \mathrm{CC}$ & & & $2-3$ & e & - & & & 할 & & e & $\Delta$ & & - & & & & & & & e & & & & & & & & & & & & \\
\hline
\end{tabular}

Figure 3. Range chart of selected Planktonic Foraminifera species at Site 251 (Holes 251 and 251 A). 


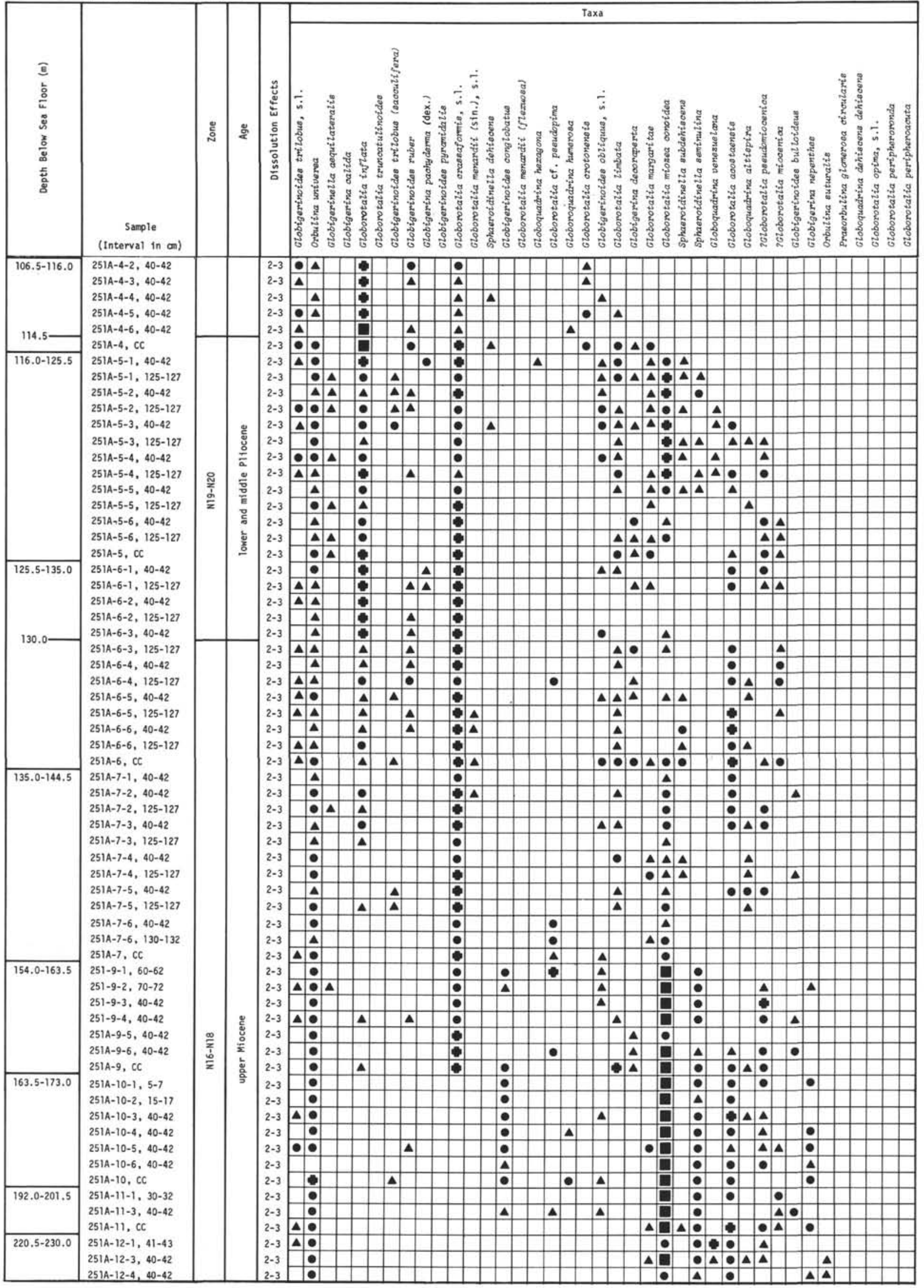

Figure 3. (Continued). 


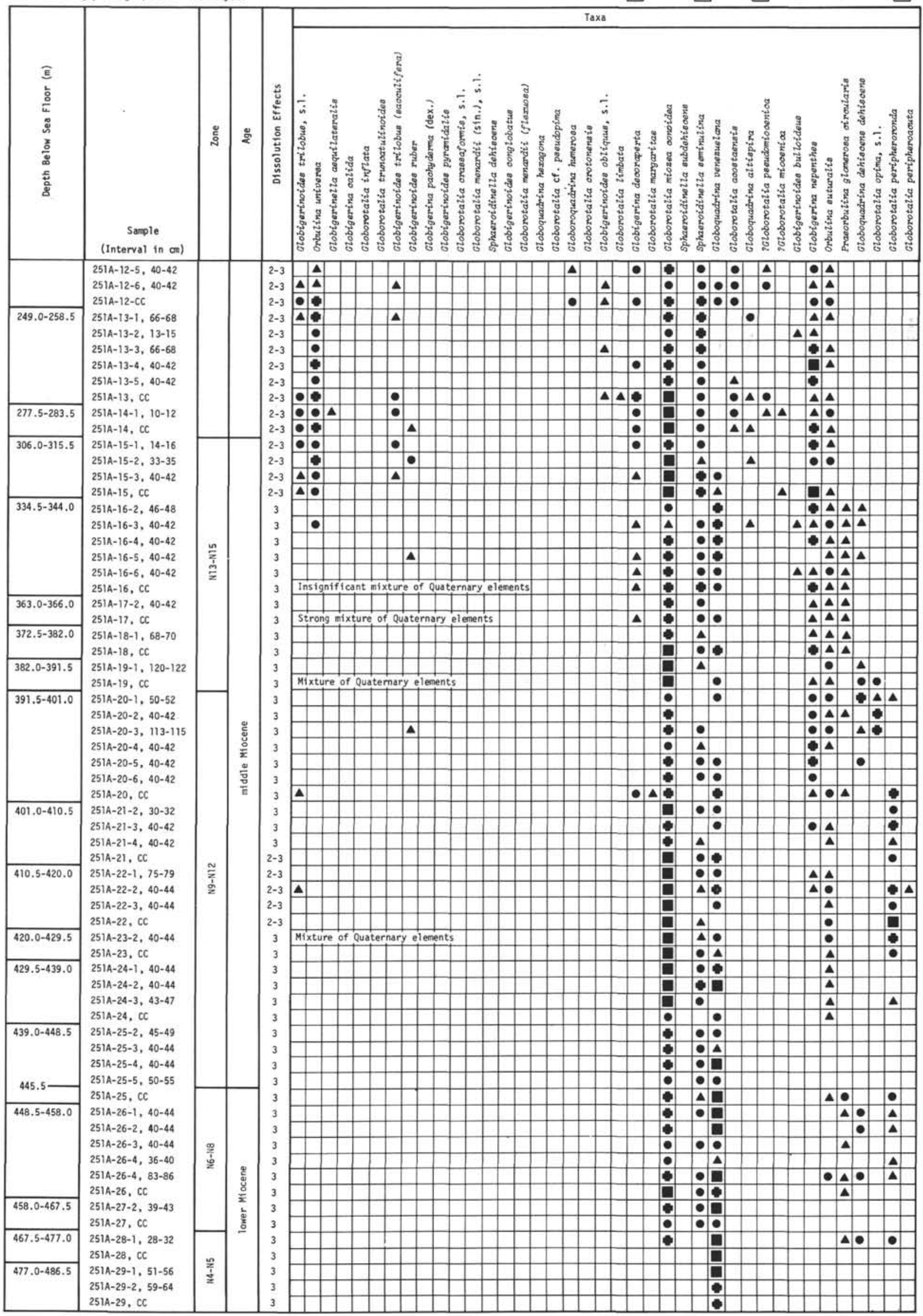

Figure 3. (Continued). 
The Quaternary/Pliocene boundary was located using the criterion of the change in ratio of Globorotalia truncatulinoides: $G$. tosaensis. In addition, this boundary is characterized by a temperature change, indicated by some increase of cold-water elements and decrease of warm-water elements in the Quaternary section as compared with the Pliocene section. The thickness of the Pliocene deposits is 93 meters.

It was possible to distinguish upper Pliocene (Zone N21); however, no paleontological evidence was found to separate the middle Pliocene (Zone N20) from the lower Pliocene (Zone N19). The upper Pliocene was detected by the first appearance of Globoquadrina humerosa. Simultaneously came Globorotalia margaritae and, somewhat lower in the section, $G$. acostaensis became extinct.

The Pliocene/Miocene boundary was located using Globorotalia inflata, s.l. as a criterion. It was rare to very rare in the upper Miocene but became frequent in the Pliocene. Other species did not show any important change in their vertical distribution across the boundary. Globorotalia crassaformis was frequent in the Pliocene and uppermost Miocene sediments. It appeared a little lower in the section than Globorotalia inflata, s.l.

Morphological changes which take place in Globorotalia inflata, s.l. are of interest and importance for stratigraphical purposes. They can be observed at other sites too, but are especially evident at Site 251 .

At this location, in the Pliocene sequence, especially in the upper Pliocene, Globorotalia inflata, s.l., is represented by typical specimens, identical to those found in the Recent oceans. In the upper Miocene section, however, it is very difficult to separate Globorotalia inflata, s.l., from G. crassaformis. Both species look very much alike and are connected by all kinds of transitional forms. Globorotalia inflata, s.l. at that time had four or even five chambers in the final whorl, a less vaulted umbilical side, flat or almost flat spiral side, and its outline was subquadrangular. On the other hand, during the same epoch many transitional forms between Globorotalia inflata, s.l., and G. miozea conoidea also existed and their separation sometimes is very difficult. This problem is discussed in detail in the section on Systematics.

The whole thickness of the Miocene deposits drilled at Site 251 is more than 350 meters. Using foraminiferal criteria, the sequence was divided into three parts: upper Miocene $(153.5 \mathrm{~m})$, middle Miocene $(161 \mathrm{~m})$, and lower Miocene $(41 \mathrm{~m})$. It was not possible to subdivide the sequence into zones.

In the upper part of the upper Miocene sequence Globorotalia crassaformis was the dominant species, but in the lower part of the upper Miocene, as well as in middle and lower Miocene sequences, Globorotalia miozea conoidea predominated.

The following criteria were used for the subdivision of the Miocene sequence. The appearance of Globorotalia limbata and $G$. acostaensis marked the middle/upper Miocene boundary. Globorotalia peripheroronda was used as an indicator of the lower/middle Miocene boundary.
The unsatisfactory state of preservation of the foraminifera at Site 251 precluded my drawing any paleoclimatological or ecological conclusions.

\section{Site 252}

Site 252 is located in the northern part of Crozet Basin. It was drilled down to 247 meters. However, only six cores were taken and these were at some distance from one other. Sediments encountered were radiolarian clay, radiolarian silty clay, clay, and diatom-bearing clay.

Evidently the sediments were deposited below the carbonate compensation depth, as almost no calcareous foraminifera were found. The few planktonic specimens found were some very rare Globorotalia inflata, s.l., recorded from a depth of 9.5 meters. According to nannoplankton data, at that depth Quaternary deposits are found.

\section{Site 253 (Figure 4)}

Site 253 was located on the southern half of the Ninetyeast Ridge. It is the northernmost site drilled during Leg 26 which contained a well-preserved fauna; 526 meters were cored; the lowermost 33 meters were partially drilled and partially cored. One meter was drilled into basalt. The Neogene sequence is 85 meters of calcareous ooze.

The foraminiferal fauna was very well preserved in Quaternary and Pliocene deposits, slightly affected by solution in the upper Miocene, and damaged in middle and lower Miocene. The whole Neogene sequence appears to have no gaps; however, I was unable, with the material available, to subdivide the sequence into zones.

The thickness of the Quaternary sediments at Site 253 is 5 meters. A study of additional samples taken at small intervals established that the Holocene sequence is only $30 \mathrm{~cm}$ thick (Boltovskoy, Late Pliocene and Quaternary paleoclimatic changes, this volume).

The Quaternary/Pliocene boundary was located by means of the Globorotalia truncatulinoides: $G$. tosaensis relationship. In addition, in the lowermost part of the Quaternary sequence, isolated Pliocene markers were recorded, namely Pulleniatina obliquiloculata praecursor.

In the Pliocene sequence Globorotalia crotonensis and, somewhat lower, G. limbata and Globigerinoides fistulosus were found.

The upper Pliocene fauna of Core 1 is underlain in Core 2 by the middle and lower Pliocene assemblages. The former is characterized by the last appearance of Sphaeroidinella seminulina, Globoquadrina venezuelana, and $G$. altispira. The lower Pliocene fauna is characterized by presence of Globorotalia margaritae.

Below the lower Pliocene upper Miocene deposits were found. The upper part of this section contained Sphaeroidinella subdehiscens, Globigerinopsis aguasayensis, Globigerinoides amplus, and some other species.

The middle Miocene was recorded in Core 7 where Globigerinoides mitra, G. bulloideus, and Globorotalia peripheroronda were found.

In the lower Miocene sequence the first occurrence (going downward) of Globigerina yeguaensis, 


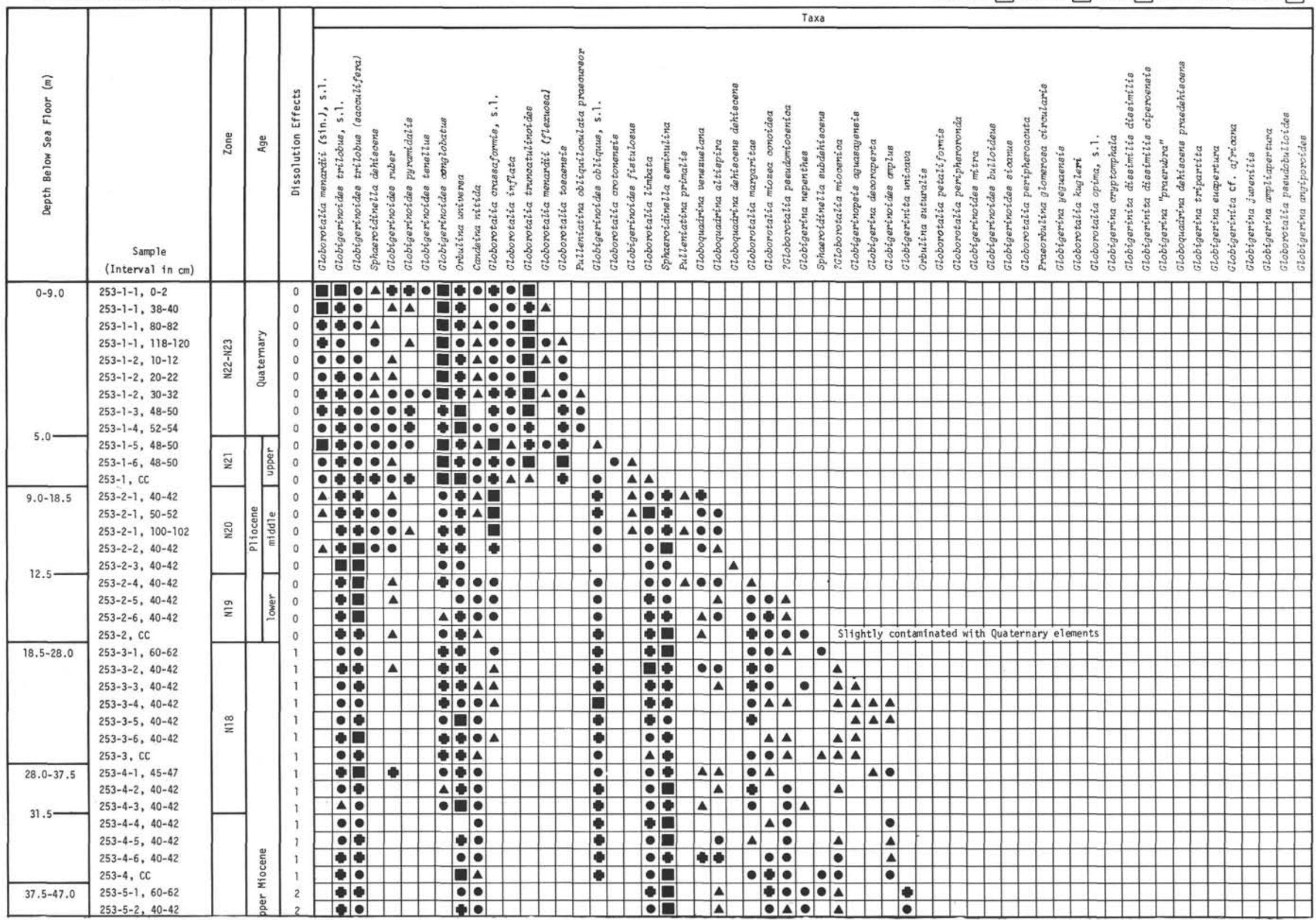

Figure 4. Range chart of selected Planktonic Foraminifera species at Site 253. 


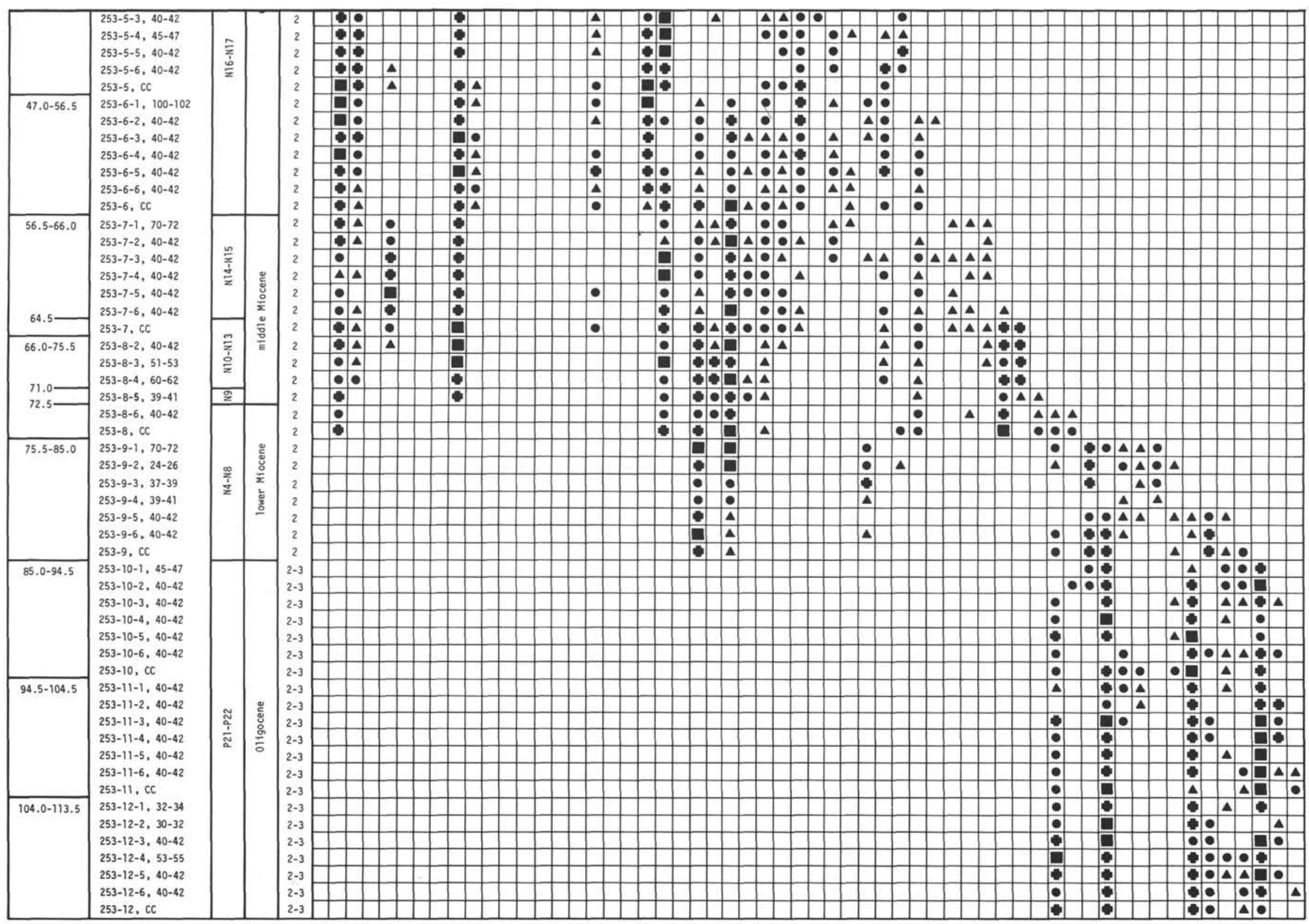

Figure 4. (Continued). 
Globorotalia kugleri, and somewhat lower, of G. opima, s.l. Globigerinita dissimilis dissimilis, $G$. dissimilis ciperoensis, and Globigerina "praerubra" were noted. In addition- the lower Miocene assemblages contained a great number of unidentifiable (usually extremely small) planktonic foraminifera.

As shown on Figure 4, at Site 253 the foraminiferal assemblages of Miocene and Oligocene are very different. The change from Oligocene to Miocene fauna is observed in about 10 meters of section, which means that it was geologically rather abrupt. To locate the boundary between Oligocene and Miocene the extinction of Globigerina ampliapertura, G. juvenilis, and Globorotalia pseudobulloides, and appearance of Globoquadrina venezuelana, $G$. dehiscens dehiscens, and Globigerina decoraperta was used.

Site 253 is a valuable site. Its shortcomings are the small thickness of the whole Neogene sequence $(85 \mathrm{~m})$ and the relatively small number of samples taken for the complete sequence, which very probably contains all the zones. Many more samples should be taken before zone boundaries can be established.

\section{Site 254 (Figure 5)}

This site is located on the Ninetyeast Ridge, approximately where the ridge joins Broken Ridge. The base of the Neogene is at a depth of 167 meters. A discontinuous Neogene sequence was recognized by means of foraminifera at this site. However, in many cases, it was not possible to separate individual zones. Fifteen samples in the Miocene section appear to be contaminated, fortunately with Quaternary specimens which were easy to separate from the in situ Miocene ones. Foraminiferal tests were well preserved throughout all the cores. Lithologically, the Neogene sequence consists mainly of coccolith-foram ooze and foram-coccolith ooze.

The Quaternary sequence is only 5.5 meters thick. The foraminiferal assemblages in it are typical of the temperate zone. Globorotalia inflata, s.l. is the dominant species and G. menardii, s.l. is found only as isolated tests. The uppermost sample, taken at a depth of $40-42$ $\mathrm{cm}$ below the bottom sample, contained rare specimens of Globorotalia tosaensis and did not have any Holocene indicators. This proves that the whole sequence is Pleistocene. If Holocene is present, it is represented by a thin layer (not thicker than $40 \mathrm{~cm}$ ).

The underlying sediments are Pliocene in age. Their age is well documented on the basis of planktonic foraminifera. The Quaternary/Pliocene boundary was located by means of the relationship between Globorotalia truncatulinoides and G. tosaensis and by the presence in the Pliocene sediments of $G$. crotonensis. The Pliocene was apparently colder; no specimens of Globorotalia menardii, s.l. were recorded.

The Pliocene sequence was divided into three parts. In the upper Pliocene $(2 \mathrm{~m})$ Globorotalia crotonensis is the characteristic species. In the middle Pliocene sequence $(7.5 \mathrm{~m})$ the extinction of Globoquadrina venezuelana and Sphaeroidinella seminulina is recorded in the uppermost part. The uppermost occurrence of Globorotalia margaritae was recorded at a depth of 15.42 meters. This is the uppermost lower Pliocene sediment.

The Pliocene/Miocene boundary was located using foraminiferal criteria. In the lowermost Pliocene Globorotalia inflata, s.l. and $G$. crassaformis were recorded frequently. In the uppermost Miocene only isolated specimens of these species were found. As at other sites the first occurrence of Globorotalia crassaformis was recorded at Site 254 a little earlier than $G$. inflata, s.l.

The Miocene sequence $(142.5 \mathrm{~m})$ is much thicker than the Pliocene. It was also divided into three parts. The extinction of Globigerinita unicava, Globigerinopsis aguasayensis, and somewhat lower of Globigerinoides sicanus and Globorotalia aff. limbata, were taken tentatively as the main criteria for locating the upper part of the middle Miocene.

The faunistical differences between middle and lower Miocene are well illustrated on the range chart (Figure 5). The extinction of such typical lower Miocene species as Globigerinita dissimilis dissimilis, $G$. dissimilis ciperoensis, Globigerina euapertura, and some others are characteristic features of the termination of that epoch.

The Miocene/Oligocene boundary is also easily observed on the same chart. Globoquadrina venezuelana and $G$. dehiscens dehiscens make their first appearance in lowermost Miocene deposits. Globigerina angulisuturalis and G.angioporoides characterize the Oligocene, although it should be noted that isolated specimens of the latter species were recorded in lower Miocene deposits too.

A great number of quite small unidentifiable planktonic foraminifera was observed in practically all the Miocene samples. Sometimes these specimens were more numerous than those which could be identified.

It is interesting to mention the relationship observed between the number of specimens of Globorotalia inflata, s.l., G. crassaformis, and G. miozea conoidea. From lower Pliocene downward the first two species decrease numerically whereas Globorotalia miozea conoidea increases. In the Miocene deposits G. inflata, s.l. and $G$. crassaformis are not found and $G$. miozea conoidea is widely distributed and numerous. It appears that the two species replace the single earlier species.

\section{Site 255 (Figure 6)}

This site is located atop Broken Ridge. It was drilled to a depth of 108.5 meters and cored almost throughout the hole; only 9.5 meters (between 13.5 and $23 \mathrm{~m}$ ) were left uncored. Unfortunately, recovery at Site 255 was very low, from 99 meters cored only 7.9 meters of core material and several core catchers were obtained. The Neogene sequence consists of a nannoplankton foraminiferal ooze. The foraminiferal tests are well preserved except in the lower Miocene material where many shells are damaged. The site is located approximately at the same latitude as Site 254 and its fauna is of the same temperate zone type. Globorotalia inflata, s.l. strongly predominates. As at Site 254, the uppermost sample (taken at depth of $4 \mathrm{~m}$ below bottom surface) contains Globorotalia tosaensis and no typical Holocene forms. Thus, it was not possible to distinguish 
Dissolution Effects (entire assemblage):

Abundance (each species):

3. Not affected; 1 - Slightly affected; 2 - Danaged;

Abundant $\square$; Common $\square$; Rare $\square$; Very rare (isolated) $\mathbf{\Delta}$

\begin{tabular}{|c|c|c|c|c|c|c|c|c|c|c|c|c|c|c|c|c|c|c|c|c|c|c|c|c|c|c|c|c|c|c|c|c|c|c|c|}
\hline & & & & & & & & & & & & & & & & & & & & & & & & axa & & & & & & & & & & & \\
\hline 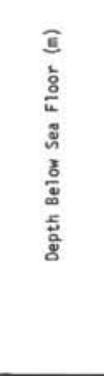 & $\begin{array}{c}\text { Sample } \\
\text { (Interval in cm) }\end{array}$ & క్ & \& & 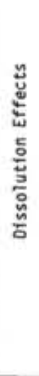 & 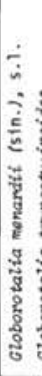 & 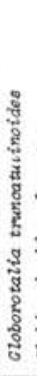 & & & 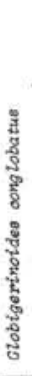 & 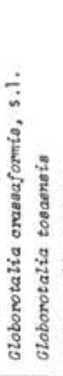 & 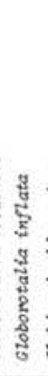 & $\begin{array}{l} \\
5 \\
5 \\
5 \\
5 \\
5 \\
5 \\
5 \\
5 \\
5 \\
5 \\
5 \\
5\end{array}$ & 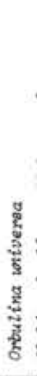 & 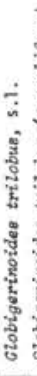 & 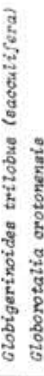 & 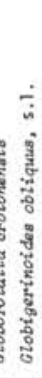 & 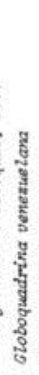 & & 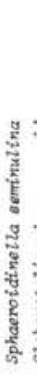 & & & 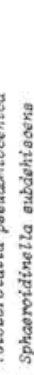 & & 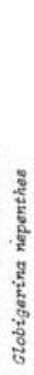 & 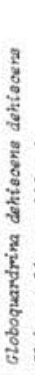 & & & 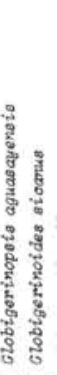 & 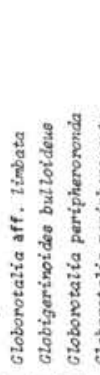 & 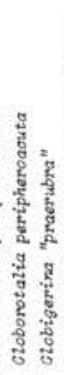 & 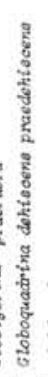 & 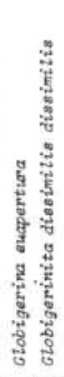 & 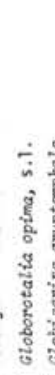 & 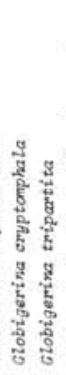 & 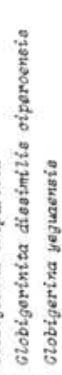 \\
\hline $0-5.5$ & $254-1-2,40-42$ & & & 0 & \begin{tabular}{|l|l}
$\Delta$ & \\
\end{tabular} & 랑 & - & $\mathbf{\Delta}$ & 항 & \begin{tabular}{l|l|}
4 \\
\end{tabular} & $\square$ & $\square$ & $\square$ & $\mathbf{\Delta}$ & - & & & & & & & & & & & & & & & & & & & & \\
\hline & $254-1-2,115-117$ & & 譥 & 0 & $\mathbf{A}$ & $\square$ & - & $\bullet$ & 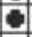 & $-\bullet$ & 0 & - & 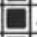 & \begin{tabular}{|c|}
$\boldsymbol{\Delta}$ \\
\end{tabular} & & & & & & & & & & & & & & & & & & & & & \\
\hline & $254-1-3,40-42$ & ָ⿳亠幺⿲丿丨丶 & E & 0 & $\Delta$ & 豆 & - & $\Delta$ & - & -6 & 司 & $\bullet$ & 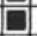 & & & & & & & & & & & & & & & & & & & & & & \\
\hline & $254-1-3,100-102$ & & ב & 0 & $\Delta$ & - & - & $\Delta$ & - & -6 & 国 & 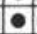 & - & 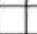 & 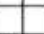 & & & & & & & & & & & & & & & & & & & & \\
\hline & $254-1, c c$ & & & 0 & $\Delta$ & 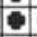 & - & - & - & - - & 담 & 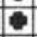 & 9 & $\bullet$ & $\mathbf{\Delta}$ & & & & & & & & & & & & & & & & & & & & \\
\hline $5.5-15.0$ & $254-2-1,40-42$ & & & 0 & \begin{tabular}{|l|l}
$\mathbf{\Delta}$ \\
\end{tabular} & $\Delta$ & $\theta$ & $\Delta$ & 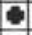 & $\square \bullet$ & {[} & 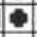 & 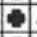 & $\Delta$ & 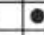 & & & & & & & & & & & & & & & & & & & & \\
\hline & $254-2-1,115-117$ & $\bar{N}$ & 屯 & 0 & & & 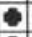 & - & - & $\square \Delta$ & $\square$ & $\bullet$ & 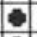 & & - & & & & & & & & & & & & & & & & & & & & \\
\hline & $254-2-2,40-42$ & $\cong$ & 흘은 & 0 & & & 8 & $\Delta$ & - & $\square$ & $\square$ & $\Delta$ & 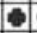 & - & & & & & & & & & & & & & & & & & & & & & \\
\hline & $254-2-2,45-47$ & & & 0 & & & - & $\Delta$ & & $\square$ & 豆 & $\bullet$ & 6 & - & - & & & & & & & & & & & & & & & & & & & & \\
\hline $1.5-$ & $254-2-3,40-42$ & & & 0 & & & $\bullet$ & & $\mathbf{\Delta}$ & $\square$ & 国 & - & 5 & $\boldsymbol{\Delta}$ & - & - & $\Delta$ & $\bullet$ & & & & & & & & & & & & & & & & & \\
\hline & $254-2-3,115-117$ & & & 0 & & & $\bullet$ & & & Q & 圆 & $\bullet$ & 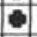 & $\bullet$ & $\Delta$ & $\Delta$ & 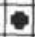 & & $\Delta$ & & & & & & & & & & & & & & & & \\
\hline & $254-2-4,40-42$ & & 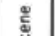 & 0 & & & $\Delta$ & & & 口 & D & $\mathbf{A}$ & $\bullet$ & - & & & - & & - & & & & & & & & & & & & & & & & \\
\hline & $254-2-4,115-117$ & & 。ั & 0 & & & $\Delta$ & & $\Delta$ & $\square \mathbf{A}$ & 回 & $\bullet$ & 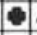 & $\Delta$ & & $\bullet$ & - & -9 & - & $\bullet$ & & & & & & & & & 1 & & & & & & \\
\hline & $254-2-5,40-42$ & జ్ి & $\bar{a}$ & 0 & & & $\Delta$ & & - & 口- & 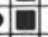 & & - & $\Delta$ & & - & 6 & $\Delta$ & - & 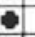 & & & & & & & & & & & & & & & \\
\hline & $254-2-5,115-117$ & & $\frac{\pi}{5}$ & 0 & & & $\square$ & & $\bullet$ & 口- & 0 & 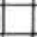 & $\bullet$ & & & $\Delta$ & - & - & - & 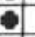 & & & & & & & & & & & & & & & \\
\hline & $254-2-6,40-42$ & & & 0 & & & - & & $\Delta$ & D & 口 & $\Delta$ & - & $\Delta$ & & $\Delta$ & & - & - & - & & & & & & & & & & & & & & & \\
\hline & $254-2-6,115-117$ & & & 0 & & & $\Delta$ & & (5) & 口内 & 国 & $\Delta$ & 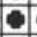 & - & - & 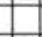 & 4 & $\mathbf{A}$ & - & 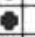 & & & & & & & & & & & & & & & \\
\hline & $254-2, c c$ & & & 0 & & & $\Delta$ & & - & 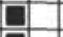 & 国 & $\bullet$ & 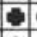 & - & - & - & $\Delta$ & - & 단 & ? & & & & & & & & & & & & & & & \\
\hline $15.0-24.5$ & $254-3-1,40-42$ & & & 0 & & & & $\Delta$ & & 口 & 6 & & $\bullet$ & $\mathbf{A}$ & & - & & $\Delta$ & - & 近 & A 1 & & & & & & & & & & & & & & \\
\hline & $254-3-1,115-117$ & & & 0 & & & $\boldsymbol{\Delta}$ & & & $\square$ & - & & 5 & & & $\bullet$ & & $\bullet$ & & 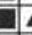 & $\Delta$ & & & & & & & & & & & & & & \\
\hline & $254-3-2,40-42$ & & & 0 & & & $\Delta$ & $\Delta$ & \begin{tabular}{|l|l|}
$\mathbf{A}$ \\
\end{tabular} & $\square$ & 8 & & ( & & & $\bullet$ & & $\Delta$ & $\Delta$ & & $\Delta \mid$ & - & & & & & & & & & & & & & \\
\hline & $254-3-2,115-117$ & & & 0 & & & & & + & $\square$ & 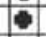 & + & 9 & & & $\Delta$ & & + & + & $\square$ & $\Delta$ & & & & & & & & & & & & & & \\
\hline & $254-3-3,40-42$ & & हु & 0 & & & & & $\Delta$ & $\square$ & 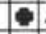 & $\Delta$ & $\bullet$ & $\Delta$ & & $\bullet$ & & $\bullet$ & & $\square$ & 1 & & & & & & & & & & & & & & \\
\hline & $254-3-3,115-117$ & & $\frac{0}{2}$ & 0 & & & & & & $\square$ & 6 & $\Delta$ & c & & & $\Delta$ & & $\bullet$ & $\Delta$ & 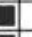 & 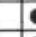 & 4 & & & & & & & & & & & & & \\
\hline & $254-3-4,40-42$ & $\bar{z}$ & & 0 & & & & & & $\square$ & 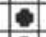 & & c & $\Delta$ & & $\bullet$ & & $\Delta$ & & , & 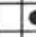 & & & & & & & & & & & & & & \\
\hline & $254-3-4,115-117$ & & 产 & 0 & & & & & - & $\square$ & 당 & & & & & - & & 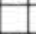 & & 包 & 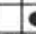 & 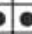 & 0 & & & & & & & & & & & & \\
\hline & $254-3-5,40-42$ & & & 0 & & & & & - & 항 & $\sqrt{-1}$ & $\boldsymbol{A}$ & - & & & - & & - & $\mathbf{A}$ & 司 & $\mathbf{A}$ & & 6 & & & & & & & & & & & & \\
\hline & $254-3-6,40-42$ & & & 0 & & & & & 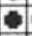 & -1 & - & & 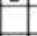 & & & $\Delta$ & & & -1 & 包 & $\Delta$ & e & 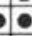 & & & & & & & & & & & & \\
\hline & $254-3-6,115-117$ & & & 0 & & & & & 달 & $\square$ & 5 & & $\bullet$ & & & $\bullet$ & & $\Delta$ & - & 0 & 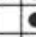 & 4 & - & - & & & & & & & & & & & \\
\hline & $254-3, \mathrm{CC}$ & & & 0 & & & & & 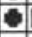 & $\square$ & - & $\Delta$ & $\varphi$ & 1 & & & & & - & ह & 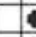 & 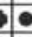 & - & $\Delta$ & & & & & & & & & & & \\
\hline $24.5-34.0$ & $254-4-1,40-42$ & & & 0 & & & & & - & $\Delta$ & $\mathbf{\Delta}$ & $\Delta$ & $\theta$ & $\Delta$ & & $\bullet$ & & & - & $\square$ & 4 & 10 & 1 & $\Delta$ & $\Delta$ & & & & & & & & & & \\
\hline & $254-4-1,115-117$ & $\stackrel{\infty}{\infty}$ & & 0 & & & & & - & & & & & & $\Delta$ & $\mathbf{A}$ & & $\Delta$ & $\bullet$ & $\square$ & 4 & 4 & 5 & 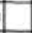 & & & & & & & & & & & \\
\hline & $254-4-2,40-42$ & $\bar{z}$ & & 0 & & & & & & $\Delta$ & & & \begin{tabular}{|c} 
\\
\end{tabular} & & & $\bullet$ & $\Delta$ & - & - & 1 & 0 & & 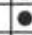 & - & & & & & & & & & & & \\
\hline & $254-4-2,115-117$ & & & 0 & & & & & & & & & D. & $\Delta$ & & & $\Delta$ & & 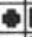 & a & 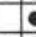 & & $\bullet$ & $\Delta$ & & & & & & & & & & & \\
\hline $28.5-$ & $254-4-3,40-42$ & & & 0 & & & & & & & & $\Delta$ & - & & & $\Delta$ & & $\Delta$ & $\Delta$ & & $\Delta$ & & - & $\Delta$ & & $\Delta$ & & & & & & & & & \\
\hline & $254-4-3,115-117$ & & & 0 & & & & & & & & & 9 & & & $\bullet$ & & $\bullet$ & - & 国 & - & & - & $\Delta$ & & 1 & & & & & & & & & \\
\hline & $254-4-4,40-42$ & & $\stackrel{\square}{E}$ & 0 & & & & & & & & & 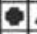 & $\Delta$ & & - & $\Delta$ & & 당 & 回 & 4 & & e & $\Delta$ & & 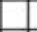 & & & & & & & & & \\
\hline & $254-4-4,115-117$ & & ष్ & 0 & & & & & & & & & 8 & & & & $\mathbf{A}$ & $\mathbf{A}$ & 8 & $\square$ & 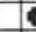 & & - & - & & & & & & & & & & & \\
\hline & $254-4-5,40-42$ & & 울 & 0 & & & & & & & & & - & & & - & $\Delta$ & - & 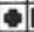 & 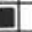 & 4 & & 6 & - & & 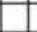 & & & & & & & & & \\
\hline & $254-4-5,115-117$ & $\hat{\approx}$ & 8 & 0 & & & & & & & & & 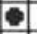 & & & $\Delta$ & - & - & - & 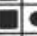 & -12 & & 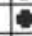 & $\Delta$ & & $\Delta$ & & & & & & & & & \\
\hline & $254-4-6,40-42$ & 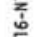 & a & 0 & & & & & & & & & - & & & $\mathbf{A}$ & - & $\bullet$ & 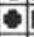 & 司 & 4 & & $\bullet$ & $\bullet$ & & $\Delta$ & & & & & & & & & \\
\hline & $254-4-6,115-117$ & $\bar{z}$ & & 0 & & & & & & & & & - & & $\Delta$ & $\mathbf{A}$ & - & $\bullet$ & 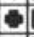 & 包 & 4 & & e & 8 & & $\Delta$ & & & & & & & & & \\
\hline & $254-4, \mathrm{CC}$ & & & 0 & & Cont: & ani & inat & tion & & & & - & $\mathbf{\Delta}$ & & & $\bullet$ & & & 包 & 8 & & $\bullet$ & 0 & & & & & & & & & & & \\
\hline $34.0-43.5$ & $254-5-1,40-42$ & & & 0 & & & & & sp & pecimen & ens & & 9 & & $\boldsymbol{\Delta}$ & & & - & 8 & 旬 & 4 & & - & $\Delta$ & & & & & & & & & & & \\
\hline & 254-5-1, 115-117 & & & 0 & & & & & & & & $\Delta$ & - & $\Delta$ & & & & - & 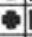 & a & 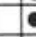 & & $\Delta$ & - & & & & & & & & & & & \\
\hline & $254-5-2,40-42$ & & & 0 & & & & & & & & & - & $\Delta$ & & & & - & 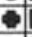 & 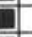 & 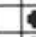 & & $\Delta$ & $\Delta$ & & & & & & & & & & & \\
\hline $36.5-$ & $254-5-2,115-117$ & & & 0 & & & & & & & & & - & - & & & & - & - & 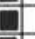 & 4 & & e & $\bullet$ & & $\Delta$ & & & & & & & & & \\
\hline & $254-5-3,40-42$ & & & 0 & & & & & & & & & - & & $\Delta$ & & & $\Delta$ & $\bullet$ & 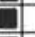 & 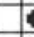 & & e & - & & $\Delta$ & 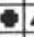 & $\Delta$ & & & & & & & \\
\hline & $254-5-3,115-117$ & & & 0 & & & & & & & & & $\bullet$ & $\Delta$ & $\Delta$ & & & $\Delta$ & $\bullet$ & 国 & 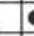 & & - & e & & $\bullet$ & 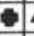 & $\Delta$ & & & & & & & \\
\hline & $254-5-4,40-42$ & & & 0 & & & & & & & & $\mathbf{\Delta}$ & - & & $\Delta$ & & & 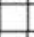 & & 曷 & 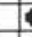 & & 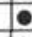 & - & & $\Delta$ & ? & & & & & & & & \\
\hline & $254-5-4,115-117$ & & & 0 & & & & & & & & & - & & & & & & 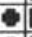 & 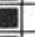 & 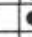 & & e & 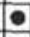 & & $\Delta$ & - & & & & & & & & \\
\hline & $254-5-5,40-42$ & & & 0 & & & & & & & & $\mathbf{A}$ & e & & & & & $\Delta$ & 8 & & 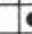 & & 단 & $\bullet$ & & - & - & & & & & & & & \\
\hline & $254-5-5,115-117$ & & & 0 & & & & & & & & & $\bullet$ & $\mathbf{\Delta}$ & & & & $\Delta$ & $\Delta$ & & 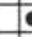 & & e & 6 & & $\Delta$ & $\bullet$ & $\Delta$ & & & & & & & \\
\hline & $254-5-6,40-42$ & & & 0 & & & & & & & & & - & - & - & & & - & - & & 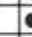 & & - & $\bullet$ & & & & $\boldsymbol{\Delta}$ & & & & & & & \\
\hline & $254-5-6,115-117$ & & & 0 & & & & & & & & & 9 & $\bullet$ & $\Delta$ & & & - & 둘 & 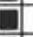 & 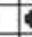 & & e & & & & $\Delta$ & & & & & & & & \\
\hline & $254-5, \mathrm{CC}$ & & & 0 & & & & & & & & & $\bullet$ & - & & & & $\bullet$ & 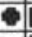 & 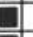 & 4 & & 6 & 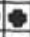 & & $\Delta$ & $\Delta$ & $\Delta$ & & & & & & & \\
\hline $43.5-53.0$ & $254-6-1,40-42$ & & & 0 & & & & & & & & & $\bullet$ & - 1 & $\boldsymbol{\Delta}$ & $\Delta$ & & - & - & 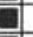 & 4 & & 4 & 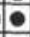 & & & - & & & & & & & & \\
\hline & $254-6-1,115-117$ & & & 0 & & & & & & & & $\Delta$ & - & - & - & & & - & 9 & 氜 & 4 & & e & 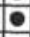 & & - & 단 & & & & & & & & \\
\hline & $254-6-2,40-42$ & & & 0 & & & & & & pecinen & ens & & - & e. & $\boldsymbol{\Delta}$ & & & & - & 旬 & 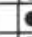 & & e & $\bullet$ & & & 든 & & & & & & & & \\
\hline & $254-6-2,115-117$ & & & 0 & & & & & & & & $\Delta$ & - & - & $\Delta$ & & & - & - & & 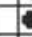 & & 6 & 5 & & & - & & & & & & & & \\
\hline & $254-6-3,20-22$ & & & 0 & & & & & & & & $\square$ & $\bullet$ & - & 단 & & & $\Delta$ & 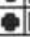 & & 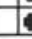 & & 6 & 6 & & \begin{tabular}{|l|l}
$\mathbf{\Delta}$ & $\mathbf{n}$ \\
\end{tabular} & & $\Delta$ & & & & & & & \\
\hline
\end{tabular}

Figure 5. Range chart of selected Planktonic Foraminifera species at Site 254. 


\begin{tabular}{|c|c|c|c|c|c|c|c|c|c|c|c|c|c|c|c|c|c|c|c|c|c|c|c|c|c|c|c|c|c|c|c|c|c|c|c|}
\hline & & & & & & & & & & & & & & & & & & & & & $\mathrm{Ta}$ & & & & & & & & & & & & & & \\
\hline 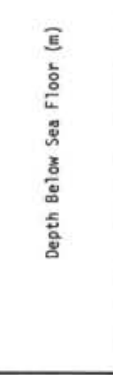 & $\begin{array}{c}\text { Sample } \\
\text { (Interval in can) }\end{array}$ & 气ूँ & $\$$ & 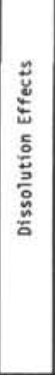 & 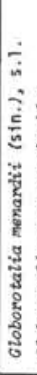 & 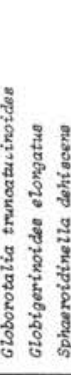 & 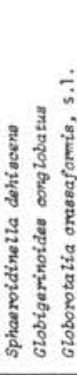 & 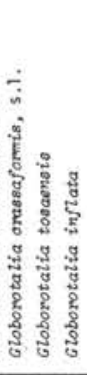 & 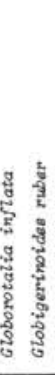 & $\begin{array}{l}0 \\
\text { है } \\
\text { है } \\
\text { है } \\
\text { है }\end{array}$ & 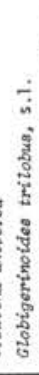 & 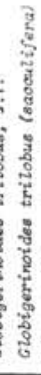 & 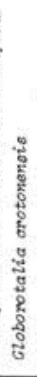 & 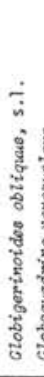 & & & & & & 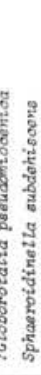 & 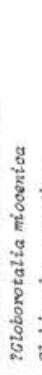 & 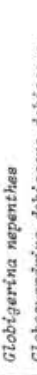 & 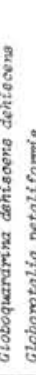 & & 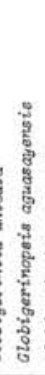 & 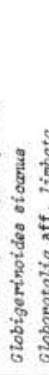 & 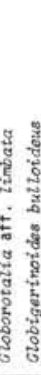 & 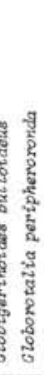 & 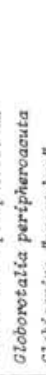 & 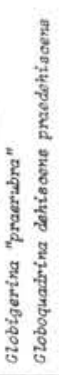 & 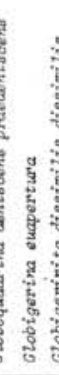 & 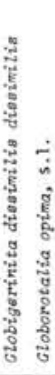 & 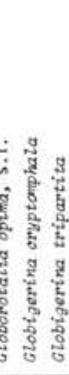 & 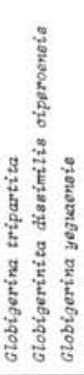 & 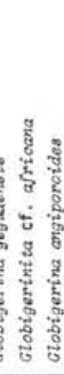 \\
\hline & $254-6-3,115-117$ & & & 0 & & contami & ination & by & & 尼 & 10 & $\mathbf{A}$ & & & & - & - [ & & 4 & & - & 8 & & (e & & $\Delta$ & & & & & & & & & \\
\hline & $254-6-4,40-42$ & & & 0 & & & nary sp & pecimens & & 불 & $\Delta$ & & & & & - & - & 司 & 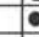 & & $\bullet$ & 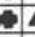 & $\Delta$ & & & $\Delta 4$ & $\mathbf{A}$ & & & & & & & & \\
\hline & $254-6-4,115-117$ & & & 0 & & & & & $\mathbf{A}$ & 0 & 4 & $\Delta$ & & $\mathbf{A}$ & & $\mathbf{A}$ & 口E & 可 & 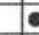 & & - & 8 & $\mathbf{A}$ & & & $\Delta$ & & & & & & & & & \\
\hline & $254-6-5,40-42$ & & & 0 & & & & & $\Delta$ & 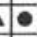 & $\Delta$ & $\Delta$ & & & $\bullet$ & - & 6 & & $\Delta$ & & $\bullet$ & - & 4 & $\Delta$ & $\bullet$ & $\Delta$ & & & & & & & & & \\
\hline & $254-6-5,115-117$ & & & 0 & & & & & $\Delta$ & (๑) & e & 10 & & $\Delta$ & - & $\Delta$ & $\bullet[$ & a & 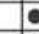 & & - & - & 8 & & & $\Delta$ & & & & & & & & & \\
\hline & $254-6-6,40-42$ & & & 0 & & & & & & 6둘 & 0 & $\bullet$ & & & - & $\Delta$ & -1 & 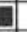 & 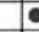 & & $\bullet$ & $\square$ & 1 & $\mathbf{\Delta}$ & & $\Delta$ & & & & & & & & & \\
\hline & $254-6-6,115-117$ & & & 0 & & & & & & 6 & $\theta$ & $\Delta$ & & & $\mathbf{\Delta}$ & - & - & 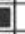 & e & & 8 & 国 & $\Delta$ & $\Delta$ & - & & & & & & & & & & \\
\hline & $254-6, C C$ & & & 0 & & & & & $\Delta$ & 6 & 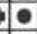 & & & & & - & - & 弱 & 4 & & 5 & D. & 5 & $\Delta$ & $\bullet$ & & & & & & & & & & \\
\hline $53.0-62.5$ & $254-7-1,40-42$ & $\cong$ & & 0 & & & & & $\boldsymbol{A}$ & 0 & e & & & & $\bullet$ & 5 & $\Delta$ & 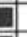 & 5 & 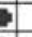 & 6 & 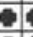 & 84 & \begin{tabular}{|l|} 
\\
\end{tabular} & $\bullet$ & & $\mathbf{\Delta}$ & & & & & & & & \\
\hline & $254-7-1,115-117$ & 耪 & & 0 & & & & & $\boldsymbol{\Delta}$ & 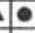 & 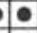 & & & & 항 & $\Delta$ & 6 & ] & e & & - & Q1 & 64 & $\mathbf{\Delta}$ & 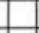 & 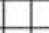 & & & & & & & & & \\
\hline & $254-7-2,40-42$ & $\bar{z}$ & & 0 & & & & & & - & $\Delta$ & & & & - & $\mathbf{\Delta}$ & $\Delta$ & 5 & 4 & & 9 & 8 & 5 & $\mathbf{\Delta}$ & $\square$ & 18 & - & & & & & & & & \\
\hline & $254-7-2,115-117$ & & & 0 & & & & & & - & $\Delta$ & & & & - & - & $\Delta$ & & $\Delta$ & 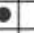 & - & $\square$ & 6 & $\mathbf{\Delta}$ & - & $\Delta \mid \mathbf{A}$ & $\mathbf{\Delta}$ & & & & & & & & \\
\hline & $254-7-3,40-42$ & & & 0 & & & & & & - & $\bullet$ & & & & - & - & 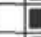 & ] & 6 & & 8 & 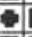 & 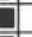 & & & & & & & & & & & & \\
\hline & $254-7-3,115-117$ & & & 0 & & & & & & $\bullet$ & & & & & - & 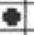 & 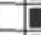 & 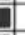 & 6 & & 단 & $\square$ & 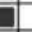 & & & & $\mathbf{\Delta}$ & & & & & & & & \\
\hline & $254-7-4,40-42$ & & & 0 & & & & & & - & & & & & 8 & 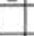 & 5 & 14 & $\Delta$ & & - & 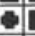 & 14 & $\Delta$ & & & & & & & & & & & \\
\hline & $254-7-4,115-117$ & & & 0 & & & & & & - & & $\mathbf{\Delta}$ & & & 8 & - & 5 & 可 & 0 & & e & -1 & 24 & $\Delta$ & & & $\mathbf{A}$ & & & & & & & & \\
\hline & $254-7-5,40-42$ & & & 0 & & & & & & $\Delta$ & $\Delta$ & & & & 8 & -5 & - 1 & 8 & e & ? & - & $\square[$ & $\square$ & & 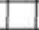 & 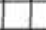 & 4 & & & & & & & & \\
\hline & $254-7-5,115-117$ & & & 0 & & & & & & $\bullet$ & & & & & ק & - & $\Delta[$ & 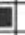 & 6 & 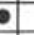 & - & $\theta$ & 8 & $\Delta$ & $\bullet$ & & 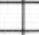 & & & & & & & & \\
\hline & $254-7-6,40-42$ & & & 0 & & & & & & - & & & & & 훌 & - & 5 & 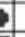 & 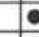 & ? & - & $\square$ & 5 & & & & \begin{tabular}{|c|}
$\mathbf{\Delta}$ \\
\end{tabular} & & & & & & & & \\
\hline & $254-7-6,115-117$ & & & 0 & & & & & & - & $\Delta$ & & & & 달 & - & 5 & 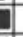 & 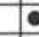 & & - & $\square$ & 4 & $\Delta$ & $\mathbf{A}$ & & 4 & & & & & & & & \\
\hline & $254-7, \mathrm{CC}$ & & & 0 & & & & & & - & 4 & & & & - & - & 6 & 百 & 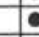 & 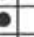 & - & 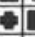 & 04 & $\Delta$ & $\bullet$ & & - & & & & & & & & \\
\hline $62.5-72.0$ & $254-8-1,40-42$ & & & 0 & & & & & $\Delta$ & 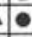 & $\Delta$ & & & & - & - & 6 & 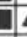 & $\Delta$ & 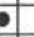 & - & 5 & 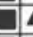 & $\Delta$ & 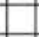 & & $\Delta$ & & & & & & & & \\
\hline & $254-8-1,115-117$ & & & 0 & & & & & & 0 & $\Delta$ & & & & e & - & - & 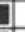 & 16 & ? & - & 8 & 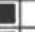 & & 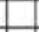 & & $\Delta \mid$ & & & & & & & & \\
\hline & $254-8-2,40-42$ & & & 0 & & & & & & - & $\Delta$ & & & & 항 & $\Delta$ & 6 & 14 & $\Delta$ & 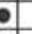 & - & - 1 & 21 & $\Delta$ & & & - & & & & & & & & \\
\hline & $254-8-2,115-117$ & & & 0 & & & & & & $\Delta$ & e & & & & - & - & 6 & 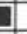 & 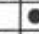 & & - & 4 & e. 4 & $\Delta$ & & & ㄹ. & & & & & & & & \\
\hline & $254-8-3,40-42$ & & & 0 & & & & & & - & $\Delta$ & & & & 6 & - & 6 & 1 & $\Delta$ & 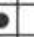 & $\bullet$ & 4 & 8 & 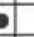 & & $\Delta$ & - & & & & & & & & \\
\hline & $254-8-3,115-117$ & & & 0 & & & & & $\mathbf{A}$ & - & 4 & & & & - & - & 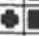 & & 6 & 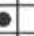 & - & क & 8 & $\Delta$ & & $\Delta$ & - & & & & & & & & \\
\hline & $254-8-4,50-52$ & & Еั & $0-1$ & & & & & & 붕 & - & & & & - & - & 6 & & & - & - & 8 & 8 & & 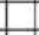 & 4 & $\Delta$ & & & & & & & & \\
\hline & $254-8-4,120-122$ & & 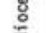 & $0-1$ & & & & & & $\mathbf{A}$ & & & & & - & - & 단 & ] & & - & - & - 1 & 8 & $\Delta$ & 品 & 4 & $\theta$ & & & & & & & & \\
\hline & $254-8-5,40-42$ & & $\frac{\bar{\Sigma}}{2}$ & $0-1$ & & & & & & - & & & & & - & $\Delta$ & 6 & ] & & • & 훙 & & 6 & ? & $\mathbf{A}$ & $\Delta$ & 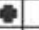 & & & & & & & & \\
\hline & $254-8-5,115-117$ & & चू & $0-1$ & & & & & & c & $\Delta$ & & & & - & - & 6 & 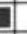 & 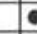 & 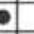 & - & t & 8 & & 8 & $\Delta$ & - 4 & & & & & & & & \\
\hline & $254-8-6,40-42$ & & 읕 & $0-1$ & & & & & & 0 & $\Delta$ & & & & 훌 & & - & & 6 & 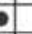 & - & 81 & 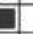 & & $\Delta$ & $\Delta$ & 8 & & & & & & & & \\
\hline & $254-8-6,115-117$ & & & $0-1$ & & & & & & 둘 & $\Delta$ & & & & 8 & $\bullet$ & $\mathbf{\Delta}$ & & 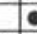 & 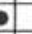 & - & 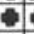 & 5 & & - & & 4 & & & & & & & & \\
\hline & $254-8, \mathrm{CC}$ & & & $0-1$ & & & & & & - & 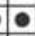 & & & & 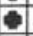 & $\Delta$ & $\Delta$ & 4 & $\Delta$ & 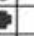 & - & $\Delta$ & 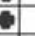 & & $\mathbf{A}$ & & - & & & & & & & & \\
\hline $72.0-81.5$ & $254-9-2,40-42$ & & & $0-1$ & & & & & & $\bullet$ & & & & & - & • & 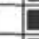 & 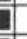 & 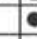 & 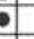 & - & 1 & 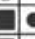 & 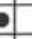 & $\Delta$ & & & & & & & & & & \\
\hline 74.5 & $254-9-2,115-117$ & & & $0-1$ & & & & & & - & & & & & - & - & L & 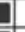 & & ? & - & $\Delta$ & 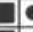 & 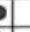 & & & & & & & & & & & \\
\hline & $254-9-3,40-42$ & & & $0-1$ & & & & & & • & & & & & 8 & - & {[} & 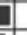 & 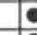 & - & क & $\Delta[$ & 14 & 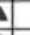 & $\mathbf{A}$ & $\Delta$ & & $\mathbf{A}$ & & & & & & & \\
\hline & $254-9-3,115-117$ & & & $0-1$ & & & & & $\Delta$ & 6 & & & & & 5 & 8 & $\Delta$ & 5 & 6 & 9 & 4 & 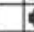 & 8 & & - & & & $\Delta$ & & & & & & & \\
\hline & $254-9, \propto$ & & & $0-1$ & & & & & & 0 & & & & & 8 & - & 6 & 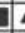 & $\Delta$ & & $\theta$ & & 8 & & + & & - & $\bullet$ & & & & & & & \\
\hline $81.5-91.0$ & $254-10-1,40-42$ & & & $0-1$ & & & & & $\Delta$ & 둘 & $\theta$ & & & & - & - & - & & & & - & [ & 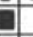 & & $\bullet$ & & 4 & 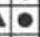 & & & & & & & \\
\hline & $254-10-1,115-117$ & & & $0-1$ & & & & & - & 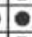 & $\sqrt{0}$ & 4 & & & - & $\bullet$ & 6 & 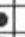 & & & - & & - 4 & 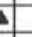 & & $\mathbf{\Delta}$ & 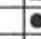 & 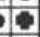 & & & & & & & \\
\hline & $254-10-2,40-42$ & & & $0-1$ & & & & & & 6 & 国 & 6 & & & - & $\mathbf{\Delta}$ & 4 & 4 & $\mathbf{A}$ & - & $\bullet$ & 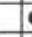 & $\bullet$ & & 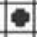 & $\Delta$ & 4 & 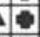 & & & & & & & \\
\hline & $254-10-2,115-117$ & & & $0-1$ & & & & & $\mathbf{\Delta}$ & - & $E$ & 10 & & & - & $\Delta$ & 86 & 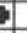 & 16 & 단 & $\Delta$ & & 3 & & - & $\Delta$ & 4 & 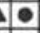 & & & & & & & \\
\hline & $254-10-3,40-42$ & & & $0-1$ & & & & & $\bullet$ & 훌 & 10 & & & & 8 & & - & 1 & $\Delta$ & - & & & 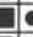 & & $\theta$ & & 6 & $\mathbf{A}$ & - & & & & & & \\
\hline & $254-10-3,115-117$ & & & $0-1$ & & & & & & e & - & 10 & & & - & & 28 & & 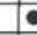 & - & & & 1 & & 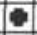 & $\Delta$ & $\Delta$ & $\Delta$ & & & & & & & \\
\hline & $254-10-4,40-42$ & & & $0-1$ & & & & & & - & 0 & - & & & - & & 24 & & 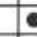 & & & 4 & 4 & & - & $\mathbf{A}$ & & - & & & & & & & \\
\hline & $254-10-4,115-117$ & & & $0-1$ & & & & & & 둥 & 들 & - & & & - & & 16 & & e & & & 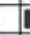 & 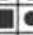 & & - & - & & - & & & & & & & \\
\hline & $254-10-5,40-42$ & & & $0-1$ & & & & & $\Delta$ & - & 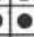 & $\theta$ & & & 훌 & & 可 & & & 5 & & 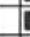 & 近 & & - & & & - & & & & & & & \\
\hline & $254-10-5,115-117$ & & & $0-1$ & & & & & $\Delta$ & 6 & 6 & 1 & & & 0 & & 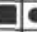 & & e & 8 & & & 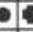 & & - & - & & ㅂ. & & & & & & & \\
\hline & $254-10-6,40-42$ & & & $0-1$ & & & & & & 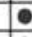 & & & & & $\varphi$ & & 2 & & 4 & 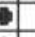 & & I & 0 & & $\Delta$ & - & & $\Delta$ & & & & & & & \\
\hline & $254-10-6,115-117$ & & & $0-1$ & & & & & & - & ? & & & $\Delta$ & 舟 & & 4 & & 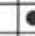 & 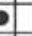 & 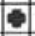 & 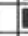 & ] & & $\bullet$ & $\varphi$ & & $\Delta$ & & & & & & & \\
\hline & $254-10, \mathrm{CC}$ & & & $0-1$ & & & & & $\Delta$ & 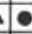 & 6 & & & & - & & 8 & & 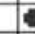 & 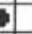 & e & 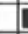 & a & & - & 8 & & $\square$ & & & & & & & \\
\hline $91.0-100.5$ & $254-11-1,115-117$ & & & $0-1$ & & & & & $\bullet$ & - & e & e & & & 6 & & 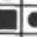 & 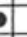 & 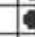 & 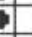 & & 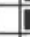 & 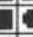 & & - & $\Delta$ & 4 & 勿 & - & & & & & & \\
\hline & $254-11-2,40-42$ & & & $0-1$ & & & & & $\Delta$ & 1 & & & & & e & & 2 & 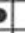 & 4 & & & 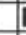 & 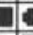 & & - & \begin{tabular}{|l|l}
$\Delta$ \\
\end{tabular} & & - & $\mathbf{A}$ & & & & & & \\
\hline & $254-11-2,115-117$ & $\stackrel{\simeq}{z}$ & & $0-1$ & & & & & & 10 & 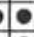 & & & & - & & 8 & 4 & 4 & & & 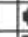 & 5 & & $\Delta$ & 01 & $\Delta$ & 당 & & & & & & & \\
\hline & $254-11-3,40-42$ & g̀ & & $0-1$ & & & & & & 0 & 1 & & & & 할 & \begin{tabular}{l|l}
$\mathbf{A}$ \\
\end{tabular} & - & 1 & $\Delta$ & & & 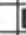 & 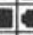 & & - & $\Delta \mathbf{A}$ & $\Delta$ & 6 & & & & & & & \\
\hline & $254-11-3,115-117$ & & & $0-1$ & & & & & & - & $\Delta$ & & & & 8 & $\Delta$ & - 4 & 4 & $\mathbf{A}$ & & & 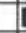 & 81 & & & $\Delta$ & & - & & & & & & & \\
\hline & $254-11-4,40-42$ & & & $0-1$ & & & & & & - & $\Delta$ & & & & 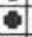 & $\Delta$ & 5 & - & 4 & & $\square$ & 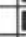 & 包 & 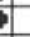 & $\bullet$ & - & & 둘 & & & & & & & \\
\hline & $254-11-4,115-117$ & & & $0-1$ & & & & & $\Delta$ & 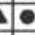 & $\theta$ & & & & - & - & -15 & 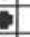 & E & ] & - & & 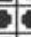 & 5 & $\bullet$ & $\mathbf{A}$ & & 9 & & & & & & & \\
\hline & $254-11-5,40-42$ & & & $0-1$ & & & & & - & e & & & & & 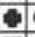 & - & 9 & 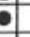 & 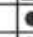 & 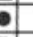 & & & 2 & 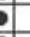 & - & $\Delta$ & & $\bullet$ & & & & & & & \\
\hline & $254-11-5,115-117$ & & & $0-1$ & & & & & & e & & & & & $\square$ & 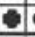 & e. & 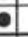 & 4 & 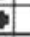 & & & 2 & 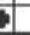 & 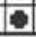 & & & $\bullet$ & & & & & & & \\
\hline
\end{tabular}

Figure 5. (Continued). 


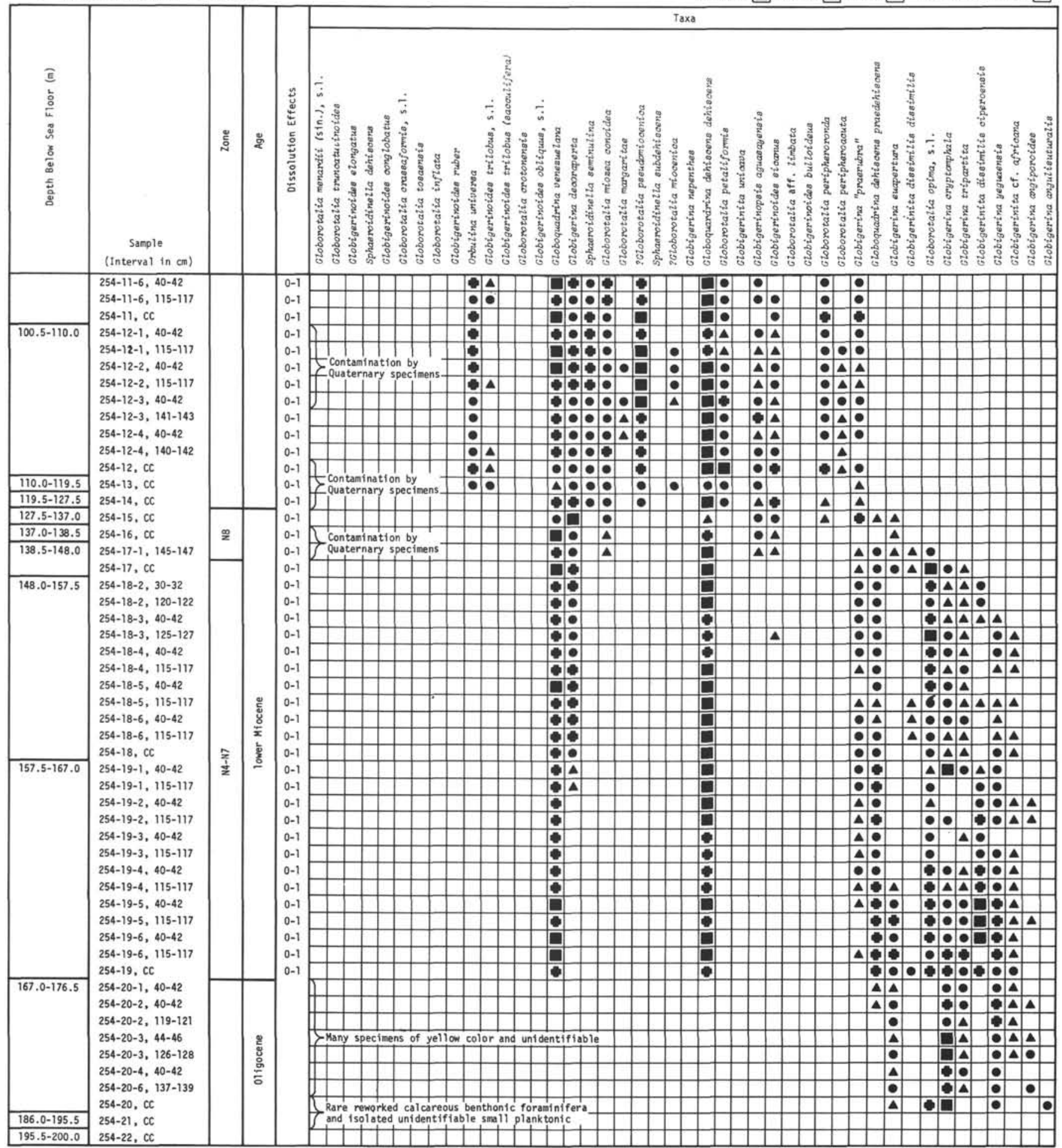

Figure 5. (Continued). 


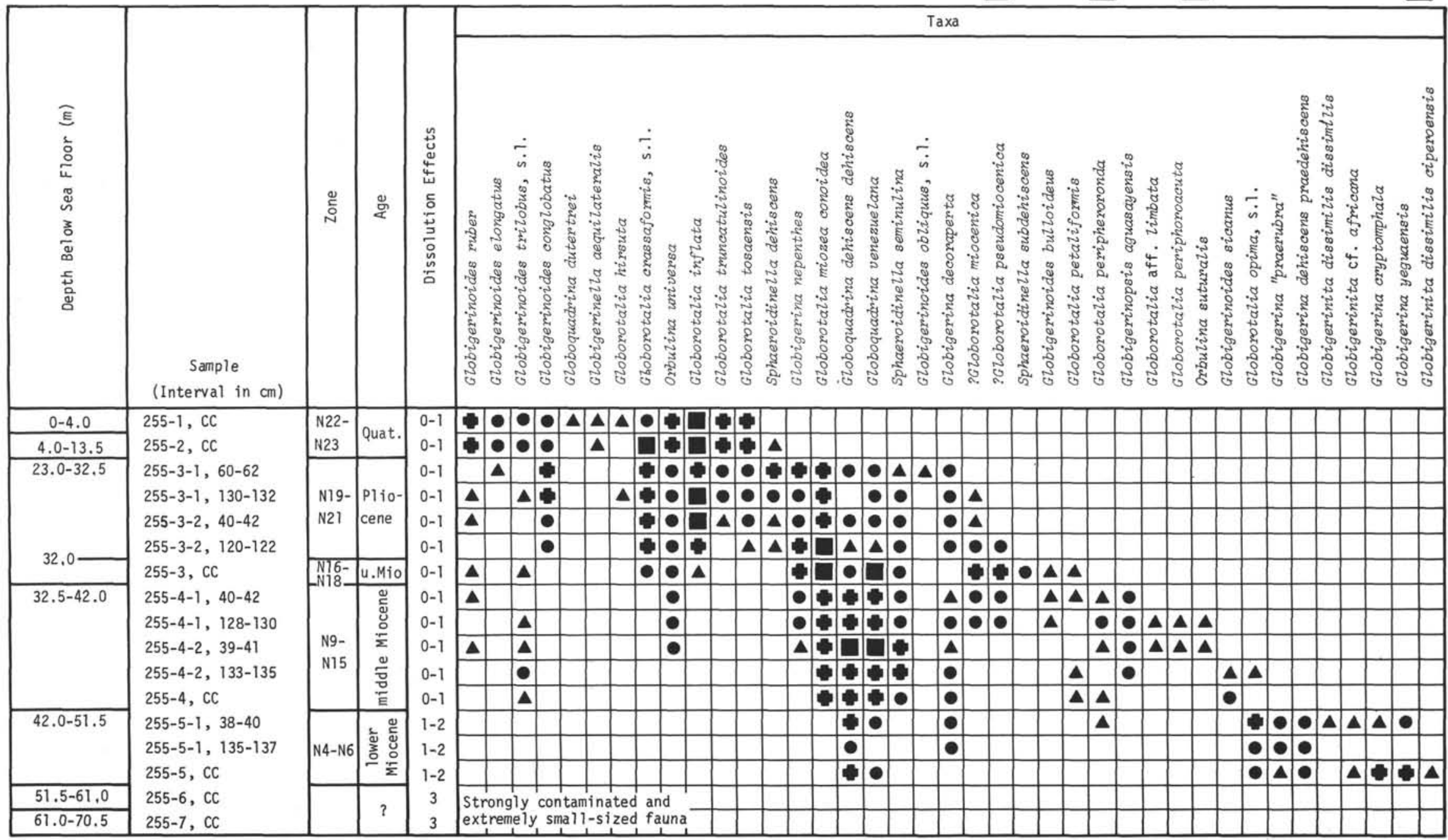

Figure 6. Range chart of selected Planktonic Foraminifera species at Site 255. 
Holocene sediments. The Holocene/Pleistocene boundary lies somewhere in the uppermost 4 meters of sediment.

The Quaternary/Pliocene boundary was determined by means of the Globorotalia truncatulinoides: G. tosaensis relationship. Also, several species (among them: Globorotalia miozea conoidea, Globigerina nepenthes, Sphaeroidinella seminulina and some others) became extinct at the end of the Pliocene. It was not possible to subdivide the Pliocene sediments.

In the Miocene sequence only upper, middle and lower Miocene were distinguished. Zones were not separated. The upper part of the lower Miocene was not detected at all.

Below the lower Miocene sediments is a sequence of unknown age. Under this sequence middle Eocene deposits were found.

\section{Site 256}

This site is situated in the southern part of Wharton Basin. It was drilled to 270 meters of which 251 meters were reddish to grayish brown clays and 19 meters basalt: 99 meters were cored. Because of the great depth the fauna was in a very bad state of preservation; many samples did not contain anything at all and several samples had either arenaceous foraminifera, which could not be used as age determinants, or rare calcareous benthonic or very small-sized unidentifiable planktonic species.

The best-preserved fauna was encountered in Sample 5-2, 35-39 cm, at a depth of 125.5 meters below the bottom surface. It contained Globorotalia miozea conoidea, G. margaritae, G. inflata, s.l., Globigerina decoraperta, G. nepenthes, G. quinqueloba, Globigerinita glutinata, and many minute unidentifiable planktonic foraminifera. Specimens were small sized, rather rare numerically, but well preserved. They did not look reworked. This assemblage can be either uppermost late Miocene or, more probably, early Pliocene in age. Thus, at least the upper 125.5 meters should be considered as Pliocene. I could not determine with the material available exactly how much of the uppermost 125.5 meters is Quaternary in age. In Sample 1-3, 40-42 cm, taken at depth of 3.5 meters down from the sea bottom, Globigerina nepenthes (one specimen) and a probable Globorotalia miozea conoidea (also one specimen) were found. Both species cannot be younger than early Pliocene in age. Sample 1, CC (depth $9.5 \mathrm{~m}$ ) contained Globigerinoides trilobus, s.l., Globorotalia inflata, s.l., and probable Globorotalia tosaensis which are also Pliocene in age. Thus, the Quarternary section is certainly less than 3.5 meters in thickness.

The sediments below the upper 125.5 meters (Pliocene-Quaternary section) were either completely barren or contained poorly preserved arenaceous foraminifera of a very wide stratigraphic range. The exact age determination of this sequence was impossible.

Based on radiolarian data, the lowermost sediments are Cretaceous in age.

The whole Neogene sequence at Site 256 undoubtedly was deposited at a depth below the carbonate compensation depth.

\section{Site 257}

This site is located in the southeastern part of the Wharton Basin. It was drilled down to a depth of 326.5 meters of which 155.5 meters were cored. Lithologically the sediments are detrital clay, clayey coccolith ooze, and chalk. As this site was below the lysocline for a long period, no calcareous fossils were found in the uppermost layers; only arenaceous benthonic foraminifera, in a rather bad state of preservation, were encountered. Specimens of Globotruncana found in Core 5 (depth 125 $\mathrm{m})$ indicate a Cretaceous age of this layer.

\section{Site 258 (Figure 7)}

Site 258 is located on the north flank of Naturaliste Plateau. Two holes were drilled: Hole 258 to 525 meters, and Hole 258A to 123.5 meters. The Neogene deposits are 114 meters of a gray-white calcareous ooze. Foraminiferal tests are very well preserved.

This is the site where the Quaternary section could be divided into Holocene and Pleistocene. For this subdivision the criterion suggested by Parker (1973) was used. The oldest sample which contained Globigerinoides ruber $(f$. rosea) is Sample 1-6, 110-112 $\mathrm{cm}$. In the same material one pink-walled Globigerina rubescens was also found. This sample was found from a depth of 8.5 meters below the bottom surface. Thus, if we accept the Parker's criterion, the uppermost 8.5 meters of Quaternary sediments are Holocene. The underlying 20.5 meters are Pleistocene. The whole thickness of the Quaternary sequence is 29 meters. Doubts with respect to validity of this criterion were discussed previously. Not being sure that the Holocene/Pleistocene boundary is located correctly, I prefer to leave it uncertain.

The Quaternary/Pliocene boundary was located utilizing the Globorotalia truncatulinoides: $G$. tosaensis ratio. As an additional criterion, the extinction of Globorotalia crotonensis and Globigerinoides obliquus, s.l. was taken.

The limit between the upper Pliocene and middle Pliocene is marked (among other things) by the extinction of Globoquadrina altispira and the appearance of Globorotalia truncatulinoides. Globorotalia tosaensis, Pulleniatina obliquiloculata, s.s.; P. obliquiloculata praecursor, and Globigerinoides pyramidalis appear in the lowermost middle Pliocene.

The most characteristic species of the lower Pliocene is Globorotalia margaritae.

For the location of the Pliocene/Miocene boundary the occurrence of Globorotalia inflata, s.l. and $G$. crassaformis was used. The sporadic records of rare or isolated specimens of the former species in the upper Miocene are in marked contrast to the abundant (or frequent) and ubiquitous appearance of specimens of the same species in the Pliocene. More or less the same phenomenon (but not as well pronounced) was observed with respect to Globorotalia crassaformis.

The following very typical upper Miocene species were encountered somewhat below the Pliocene/Miocene boundary: Orbulina suturalis, Globigerinoides amplus, Globigerinita unicava, and Globorotalia petaliformis. 


\begin{tabular}{|c|c|c|c|c|c|c|c|c|c|c|c|c|c|c|c|c|c|c|c|c|c|c|c|c|c|c|c|c|c|c|c|c|}
\hline & & & & & & & & & & & & & & & & & & & & & Ta & xa & & & & & & & & & & \\
\hline 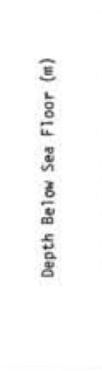 & $\begin{array}{c}\text { Sample } \\
\text { (Interval in cm) }\end{array}$ & క్ & ๕ & 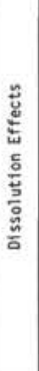 & & 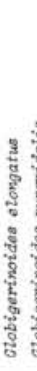 & 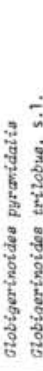 & 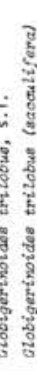 & & & 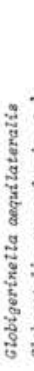 & & & 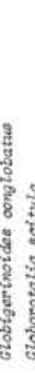 & & & & 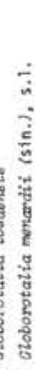 & & 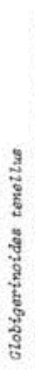 & 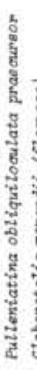 & & 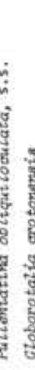 & & & & & & 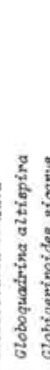 & 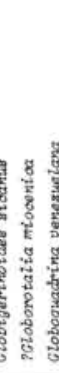 & 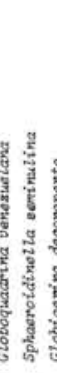 & 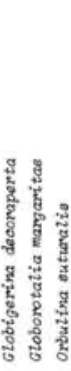 \\
\hline $0-9.5$ & $258-1-1,20-22$ & & & 0 & - & - & $\Delta$ & 04 & 10 & 1 & \begin{tabular}{|l|l}
$\Delta$ \\
\end{tabular} & - & 04 & $\mathbf{\Delta}$ & $\Delta$ & 5 & & & & & & & & & & & & & & & & \\
\hline & $258 A-1-1,20-22$ & & & 0 & - & - & $\Delta$ & - $\Delta$ & $\bullet$ & - & $\Delta$ & - & - 4 & $\Delta$ & 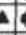 & . & & & & & & & & & & & & & & & & \\
\hline & $258 \mathrm{~A}-1-1,37-39$ & & & 0 & \begin{tabular}{|c|}
$\mathbf{A}$ \\
\end{tabular} & $\Delta$ & & & $\Delta$ & $\bullet$ & 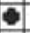 & & 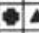 & $\mathbf{A}$ & 0 & 7 & 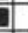 & & & & & & & & & & & & & & & \\
\hline & $258 A-1-1,61-63$ & & & 0 & - & $\mathbf{\Delta}$ & $\Delta$ & $\Delta$ & $\bullet$ & -1 & - & & - & & , & 5 & & & & & & & & & & & & & & & & \\
\hline & $258-1-1,80-82$ & & & 0 & 항 & - & 1 & $\Delta 4$ & $\Delta$ & & - & - & & 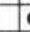 & 0 & 5 & . & D & & & & & & & & & & & & & & \\
\hline & $258 \mathrm{~A}-1-1,138-140$ & & & 0 & - & $\mathbf{A}$ & & & & $\bullet$ & - & $\mathbf{\Delta}$ & 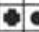 & - & $\Delta$ & 5 & 4 & $\Delta$ & & & & & & & & & & & & & & \\
\hline & $258-1-2,20-22$ & & & 0 & - & - & & 4 & $\Delta$ & & - & - & & & $\bullet$ & 1 & 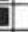 & & & & & & & & & & & & & & & \\
\hline & $258 \mathrm{~A}-1-2,70-72$ & & & 0 & - & - & $\Delta$ & 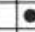 & & & - & $\bullet$ & & & $\Delta$ & 5 & 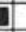 & - & & & & & & & & & & & & & & \\
\hline & $258-1-2,73-75$ & & Е & 0 & $\bullet$ & & & & & & $\Delta$ & - & & 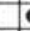 & ( & $\bullet$ & 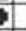 & $\Delta$ & & & & & & & & & & & & & & \\
\hline & $258 A-1-2,100-102$ & & ¿ & 0 & $\Delta$ & $\Delta$ & & 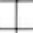 & 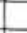 & & $\Delta$ & $\mathbf{\Delta}$ & 1 & 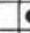 & $\bullet$ & - & 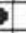 & $\Delta$ & & & & & & & & & & & & & & \\
\hline & $258 A-1-2,133-135$ & & 홍 & 0 & $\Delta$ & & & 1 & 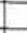 & & - & $\mathbf{A}$ & $\bullet$ & & 5 & 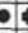 & 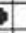 & 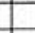 & $\Delta$ & & & & & & & & & & & & & \\
\hline & $258-1-3,25-27$ & & & 0 & (6) & & 4 & $\Delta \Delta$ & $\Delta$ & & - & $\bullet$ & - 1 & $\Delta$ & 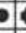 & 1 & 7 & ? & & & & & & & & & & & & & & \\
\hline & $258-1-3,100-102$ & & & 0 & 당 & & e & e 4 & & & - & - & $\Delta$ & & e & 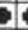 & 1 & 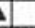 & 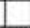 & & & & & & & & & & & & & \\
\hline & $258-1-4,20-22$ & & & 0 & - & & & $\Delta$ & $\bullet$ & & - & $\bullet$ & & 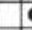 & 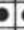 & a & 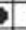 & $\Delta$ & $\Delta$ & $\mathbf{A}$ & & & & & & & & & & & & \\
\hline & $258-1-4,108-110$ & & & 0 & - & & & $\Delta 4$ & $\Lambda$ & & - & $\bullet$ & & 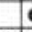 & 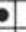 & & 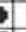 & $\Delta$ & & & $\Delta$ & & & & & & & & & & & \\
\hline & $258-1-5,40-42$ & & & 0 & - & $\boldsymbol{A}$ & 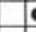 & 0 & 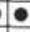 & & - & $\bullet$ & & $\Delta$ & $\Delta$ & & 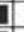 & 4 & & & & & & & & & & & & & & \\
\hline & $258-1-5,112-114$ & & & 0 & - & & & $\Delta \mathbf{4}$ & 0 & & - & - & & $\Delta$ & $\Delta$ & - 1 & ] & $\bullet$ & $\Delta$ & & & & & & & & & & & & & \\
\hline & $258-1-6,25-27$ & & & 0 & 당 & 1 & & 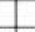 & - & & - & - & 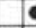 & - & $\Delta$ & e[ & ] & $\bullet$ & $\Delta$ & & & - 14 & $\Delta$ & & & & & & & & & \\
\hline $8.5-$ & $258-1-6,110-112$ & $\stackrel{N}{\dot{\pi}}$ & $?-$ & 0 & - & $\bullet$ & & - 4 & $\bullet$ & & $\Delta$ & - & - & & - & e] & ] & e & & - & & & & & & & & & & & & \\
\hline & $258-1, \mathrm{CC}$ & $\mathbb{Z}$ & & 0 & $\bullet$ & e & & $\Delta$ & $\bullet$ & & - & $\mathbf{A}$ & $\Delta$ & & 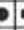 & 6 & 6 & - & & & & - & & & & & & & & & & \\
\hline & $258 \mathrm{~A}-1, \mathrm{CC}$ & & & 0 & - & - & & $\mathbf{A}$ & $\bullet$ & & - & \begin{tabular}{c|c} 
\\
\end{tabular} & $\mathbf{A}$ & 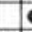 & $\bullet$ & 5 & 6 & 10 & & & & & & & & & & & & & & \\
\hline $9.5-19.0$ & $258-2-1,88-90$ & & & 0 & 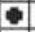 & & 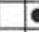 & e & $\Delta$ & & - & $\mathbf{\Delta}$ & & $\Delta$ & $\Delta$ & e] & 12 & 0 & & & & 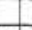 & & & & & & & & & & \\
\hline & $258-2-2,30-32$ & & & 0 & - & & $\Delta$ & 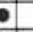 & $\Delta$ & & - & $\mathbf{A}$ & & $\mathbf{\Delta}$ & & - [ & 2 & $\bullet$ & & & & $\Delta$ & & & & & & & & & & \\
\hline & $258-2-2,90-92$ & & & 0 & - & & & e & $\Delta$ & & e & $\mathbf{A}$ & & $\Delta$ & 0 & 5 & 1 & - $\Delta$ & & & & & & & & & & & & & & \\
\hline & $258-2-3,30-32$ & & & 0 & - & & 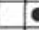 & 4 & $A$ & & - & $\Delta$ & & 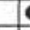 & | & ] [ & 1 & $\Delta$ & & & & & & & & & & & & & & \\
\hline & $258-2-3,115-117$ & & & 0 & $\Delta$ & & 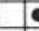 & e & $\Delta$ & & - & $\Delta$ & & & A & e. & 14 & $\Delta$ & & & & & & & & & & & & & & \\
\hline & $258-2, \mathrm{CC}$ & & ๕ั้ & 0 & $\Delta$ & $\bullet$ & $\Delta \mathbf{A}$ & $\Delta \mathbf{A}$ & $\Delta$ & & - & 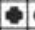 & - 2 & $\mathbf{\Delta}$ & $\Delta$ & e1 & 10 & 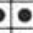 & & & & & & & & & & & & & & \\
\hline $19.0-28.5$ & $258 \mathrm{~A}-2-1,0-135$ & & ๕ัّ & 0 & - & & & $\Delta \mathbf{\Delta}$ & & & - & & $\bullet$ & $\Delta$ & 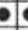 & 0 & ] & $\Delta$ & & & & & & & & & & & & & & \\
\hline & $258 \mathrm{~A}-2-1,138-140$ & & $\frac{\pi}{6}$ & 0 & $\Delta$ & - & & $\Delta \mathbf{\Delta}$ & $\Delta$ & & $\Delta$ & - & 6 & $\mathbf{\Delta}$ & & e] & 7 & $\Delta$ & & & & & & & & & & & & & & \\
\hline & $258 A-2-2,3-5$ & & $\frac{0}{2}$ & 0 & $\bullet$ & - & & $\mathbf{A} \mathbf{A}$ & $\bullet$ & & - & $\bullet$ & $\bullet$ & $\Delta$ & $\bullet$ & 5 & 7 & 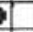 & & & & & 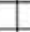 & & & & & & & & & \\
\hline & $258 A-2-2,110-112$ & & & 0 & - & $\Delta$ & & $\mathbf{A}$ & & & - & $\bullet$ & $\bullet$ & 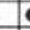 & 5 & e] & Te & $\Delta$ & & & & & & & & & & & & & & \\
\hline & $258 \mathrm{~A}-2-2,130-150$ & & & 0 & $\Delta$ & & & $\mathbf{A}$ & 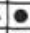 & & $\Delta$ & - & $\bullet$ & $\Delta$ & & 1 & 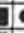 & $\Delta$ & & & & & & & & & & & & & & \\
\hline & $258 \mathrm{~A}-2-3,8-10$ & & & 0 & & A & & & $\Delta$ & & $\Delta$ & $\bullet$ & $\Delta$ & 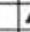 & $\Delta$ & 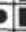 & 7 & $\Delta$ & & & & & & & & & & & & & & \\
\hline & $258 \mathrm{~A}-2-3,20-150$ & & & 0 & $\bullet$ & $\Delta$ & $\Delta$ & $\boldsymbol{\Delta}$ & $\Delta$ & & & - & $\mathbf{\Delta}$ & 4 & $\Delta$ & {[} & 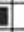 & $\Delta$ & & & & & & & 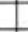 & & & & & & & \\
\hline & $258 \mathrm{~A}-2, \mathrm{CC}$ & & & 0 & - & $\Delta$ & - & $\boldsymbol{\Delta}$ & $\Delta$ & & & - & - & - & $\Delta$ & -1 & 6 & $\Delta$ & & & & & & & & & & & & & & \\
\hline $28.5-38.0$ & $258 A-3-1,46-48$ & & & 0 & - & $\Delta$ & $\Delta$ & $\Delta \mathbf{A}$ & 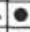 & & & 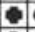 & e. & - & $\Delta$ & $\Delta$ & 14 & 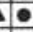 & & & & & & & & & & & & & & \\
\hline 29.0 & $258 A-3-1,67-69$ & & & 0 & - & & 1 & $\mathbf{A} \mathbf{A}$ & 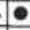 & & & 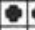 & 02 & $\mathbf{\Delta}$ & $\Delta$ & $\Delta$ & 14 & 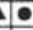 & & & & & & & & & & & & & & \\
\hline & $258 \mathrm{~A}-3-2,15-17$ & & & 0 & $\Delta$ & $\bullet$ & - & $\mathbf{\Delta} \mathbf{\Delta}$ & $\Delta$ & & - & - & - & - & • & a & 14 & $\Delta$ & & & & 4 & $\Delta$ & 5 & $\Delta$ & - & & & & & & \\
\hline & $258 A-3-2,45-47$ & & & 0 & $\mathbf{A}$ & - & & & & & $\Delta$ & - & -1 & - & $\Delta$ & t & 7 & $\Delta$ & & & & & 1 & • & & & & & & & & \\
\hline & $258 A-3-2,75-77$ & & & 0 & e & $\bullet$ & 8 & e & 4 & & $\mathbf{A}$ & 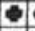 & -1 & - & 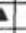 & & 7 & $\Delta$ & & & $\Delta$ & & 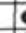 & 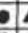 & $\Delta$ & & & & & & & \\
\hline & $258 A-3-2,97-99$ & & & 0 & - & - & 18 & $\Delta$ & & & & 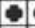 & - 2 & $\Delta$ & $\Delta$ & & 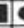 & ? & & & & & & - & e & & & & & & & \\
\hline & $258 \mathrm{~A}-3-2,131-133$ & & & 0 & & & \begin{tabular}{|c|c|} 
\\
\end{tabular} & & $\Delta$ & & $\Delta$ & 항 & - & & & $\Delta[$ & & 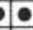 & & & & & 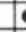 & • & & & & & & & & \\
\hline & $258 A-3-3,15-17$ & & & 0 & & $\mathbf{A}$ & $\Delta$ & e & & & $\Delta$ & - & - & & $\Delta$ & & & 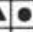 & & & & & & 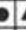 & $\Delta$ & & & & & & & \\
\hline & $258 A-3-3,45-47$ & & & 0 & $\Delta$ & - & $\Delta$ & - & $\Delta$ & & $\Delta$ & 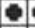 & 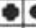 & - & - & 1 & & $\Delta$ & & & & & 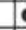 & - & - & & & & & & & \\
\hline & $258 \mathrm{~A}-3-3,75-77$ & & & 0 & $\Delta$ & - & - & & & & e & - & 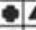 & $\mathbf{\Delta}$ & 5 & $\Delta$ & & $\Delta$ & & & & & 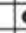 & $\cdot 1$ & $\Delta$ & 2 & & & & & & \\
\hline & $258 A-3-3,105-107$ & & & 0 & - & $\Delta$ & $\Delta$ & $\Delta 4$ & 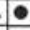 & & & - & 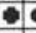 & - & . & $\Delta$ & 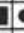 & - & & & 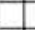 & & 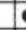 & - & & & & & & & & \\
\hline & $258 \mathrm{~A}-3-3,135-137$ & & & 0 & $\bullet$ & $\Delta$ & 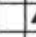 & $\Delta$ & $\bullet$ & & & - & 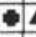 & $\Delta$ & 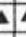 & $\mathbf{A}$ & 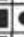 & $\Delta$ & & & & $\mathbf{\Delta}$ & & & & & & & & & & \\
\hline & $258 \mathrm{~A}-3-4,20-22$ & & & 0 & & - & s & - 4 & & & $\Delta$ & 당 & - & - 1 & $\Delta$ & ] & 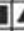 & 10 & & & & & 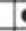 & - & $\mathbf{A}$ & & & & & & & \\
\hline & $258 A-3-4,45-47$ & & & 0 & $\Delta$ & - & T & - 4 & $\Delta$ & & $\Delta$ & 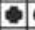 & - & - & $\Delta$ & 1 & & e & & & & & & $\bullet$ & $\Delta$ & & & & & & & \\
\hline & $258 \mathrm{~A}-3-4,79-81$ & & & 0 & & $\Delta$ & \begin{tabular}{|l|l}
$\mathbf{A}$ & $\mathrm{s}$ \\
\end{tabular} & $\Delta$ & & & 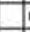 & - & & $\mathbf{\Delta}$ & & 5 & 8 & 50 & & & & & 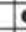 & 0 & t. & & & & & & & \\
\hline & $258 \mathrm{~A}-3-4,110-112$ & & & 0 & & & 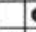 & $\bullet 4$ & $\Delta$ & & $\Delta$ & 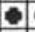 & -1 & e. & $\Delta$ & I & 1 & 10 & & & & & & & & & & & & & & \\
\hline & $258 A-3-4,136-138$ & & & 0 & & $\mathbf{A}$ & 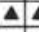 & $\Delta$ & 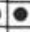 & & & 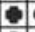 & - & e. & e. & 1 & $\sqrt{6}$ & e & & & $\Delta$ & & & 1 & a) & & & & & & & \\
\hline & $258 \mathrm{~A}-3-5,15-17$ & & & 0 & $\Delta$ & - & 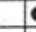 & 0 & $\Delta$ & & $\Delta$ & - & $\bullet$ & • 1 & $\Delta$ & [ & 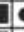 & e & & & & & 4 & $\Delta$ & $\Delta$ & & & & & & & \\
\hline & $258 A-3-5,45-47$ & & & 0 & $\Delta$ & - & 8 & 9 & $\Delta$ & & $\Delta$ & 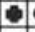 & - & $\mathbf{\Delta}$ & 1 & a & 16 & e & & & & & & 1 & 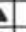 & 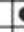 & & & & & & \\
\hline & $258 A-3-5,75-77$ & & & 0 & $\Delta$ & $\Delta$ & 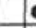 & - 4 & $\Delta$ & & - & & - & & 4 & $\Delta$ & c & 4 & & & $\Delta$ & & 1 & A 2 & $\Delta$ & 2 & & & & & & \\
\hline & $2584-3-5,107-109$ & & & 0 & - & $\bullet$ & 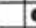 & 24 & & & $\Delta$ & & - & & & a & ] & $\bullet$ & & & & & $\Delta$ & & & & & & & & & \\
\hline & $258 a-3-5,115-117$ & & & 0 & & - & - & 0 & $\Delta$ & & $\Delta$ & $\Delta$ & - & $\Delta$ & of & L & ]e & 6 & & & & & $\Delta$ & 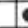 & - & 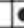 & & & & & & \\
\hline & $258 A-3-5,134-136$ & & ๕ & 0 & & - & $\Delta$ & 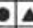 & $\Delta$ & & $\Delta$ & $\Delta$ & 6 & 2 & $\Delta$ & - & ] & 6 & & & $\Delta$ & & $\Delta$ & & & 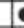 & & & & & & \\
\hline & $258 A-3-6,15-17$ & & ه్ & 0 & $\bullet$ & - & \begin{tabular}{|c|c} 
\\
\end{tabular} & 4 & (e & - & $\Delta$ & - & & $\Delta$ & $\Delta$ & 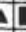 & 5 & 6 & & & & & & 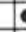 & - & 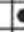 & 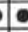 & & & & & \\
\hline & $258 \mathrm{~A}-3-6,45-47$ & 폻 & $\frac{1}{2}$ & 0 & $\bullet$ & - & T & 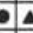 & & $\Delta$ & $\Delta$ & $\Delta$ & - & $\Delta$ & $\Delta$ & {[} & e & e & & & & & & & & & 6 & & & & & \\
\hline & $258 A-3-6,75-77$ & & E & 0 & - & - & 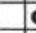 & 0 & $\Delta$ & & & - & - & 4 & $\Delta$ & a & 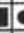 & 4 & & & & & & 2 & $\Delta$ & 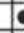 & 6 & & & & & \\
\hline & $258 A-3-6,105-107$ & & $\frac{a}{3}$ & 0 & & $\Delta$ & 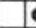 & - 4 & - & & - & $\Delta$ & - & $\Delta$ & 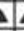 & $\Delta$ & & $\Delta$ & & & & & & & & & 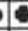 & & & & & \\
\hline & $258 \mathrm{~A}-3-6,135-137$ & & & 0 & & - & 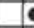 & 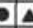 & 0 & - & - & $\Delta$ & -1 & - & t & at & & 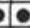 & & & & & & & $\Delta$ & & 6 & & & & & \\
\hline & $258 \mathrm{~A}-3, \mathrm{CC}$ & & & 0 & & $\bullet$ & D & 4 & $\Delta$ & $\Delta$ & $\Delta$ & $\Delta$ & e. & - & D & e[ & & 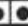 & & & & & & & $\mathbf{a}$ & & 1 & 1 & & & & \\
\hline
\end{tabular}

Figure 7. Range chart of selected Planktonic Foraminifera species at Site 258 (Holes 258 and 258A). 


\begin{tabular}{|c|c|c|c|c|c|c|c|c|c|c|c|c|c|c|c|c|c|c|c|c|c|c|c|c|c|c|c|c|}
\hline & & & & & & & & & & & & & & & & & & $\mathrm{Ta}$ & & & & & & & & & & \\
\hline 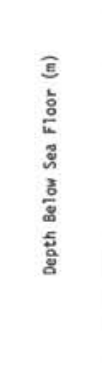 & $\begin{array}{c}\text { Sample } \\
\text { (Interval in cal) }\end{array}$ & 气ूँ & \& & 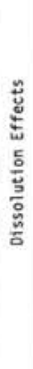 & 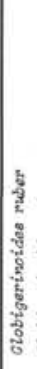 & 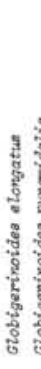 & 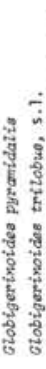 & 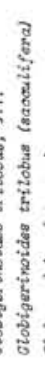 & & 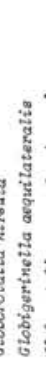 & 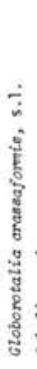 & 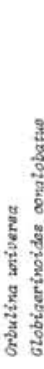 & & 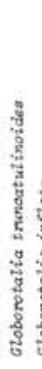 & & 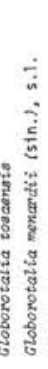 & 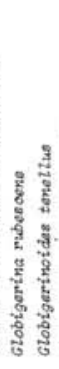 & 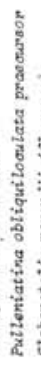 & 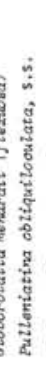 & & & 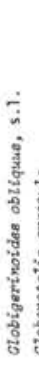 & 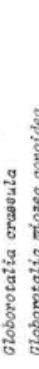 & & & 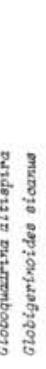 & 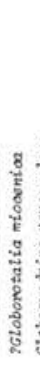 & \\
\hline $38.0-47.5$ & $258 \mathrm{~A}-4-1,120-122$ & & & 0 & $\bullet$ & $\bullet$ & 6 & & - & $\Delta$ & 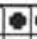 & 14 & $\mathbf{A} \cdot$ & $\bullet$ & 口e & $\bullet \bullet$ & & & $\Delta$ & & $\Delta$ & & - & & & & & \\
\hline & $258 A-4-2,15-17$ & & & 0 & $\bullet$ & $\bullet$ & e & & - & $\Delta$ & - & 4 & $\bullet$ & $\bullet$ & Q4 & $\Delta \bullet$ & & & - & & $\square$ & & . & - & & & & \\
\hline & $258 A-4-2,45-47$ & & & 0 & $\bullet$ & $\Delta$ & $\Delta$ & & $\Delta$ & - & $\mathbf{A}$ & $\varphi$ & $\bullet$ & -1 & 口 & $\bullet$ & & & $\Delta$ & & $\Delta$ & & 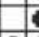 & 단 & & & & \\
\hline & $258 A-4-2,75-77$ & & & 0 & $\Delta$ & \begin{tabular}{|l|l}
$\Delta$ & 1 \\
\end{tabular} & $\Delta \bullet$ & $\Delta$ & - & $\bullet$ & $\mathbf{A}$ & - & $\Delta$ & $\Delta$ & 口e & -10 & & & $\Delta$ & & $\bullet$ & & 6 & - & & & & \\
\hline & $258 A-4-2,105-107$ & & & 0 & $\mathbf{A}$ & $\bullet$ & 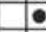 & & $\bullet$ & $\bullet$ & & - & & $\bullet$ & 回 & - $\bullet$ & & $\Delta$ & $\Delta$ & & $\bullet$ & & 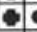 & e & & & & \\
\hline & $258 A-4-2, \quad 137-139$ & & & 0 & $\bullet$ & - & - & 4 & $\bullet$ & $\bullet$ & 9 & - & $\Delta$ & $\mathbf{\Delta}$ & 口- & $\bullet$ & & & & & 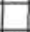 & & $\Delta$ & 8 & & & & \\
\hline & $2584-4-3,15-17$ & & & 0 & & & - & $\Delta$ & - & - & $\Delta$ & 4 & $\mathbf{A} \mathbf{A}$ & $\Delta$ & $\square$ & - $\bullet$ & & 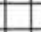 & 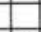 & & $\Delta$ & & - 2 & $\Delta$ & & & & \\
\hline & $258 A-4-3,45-47$ & & & 0 & $\bullet$ & $\bullet$ & $\Delta$ & & $\bullet$ & $\Delta$ & $\bullet$ & 94 & $\Delta \mathbf{A}$ & $\Delta$ & 口e & $\bullet$ & & 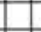 & $\Delta$ & & & & - & 8 & & & & \\
\hline & $258 A-4-3,105-107$ & & & 0 & - & $\bullet$ & \begin{tabular}{|l|l}
$\mathbf{A}$ & $\mathbf{A}$ \\
\end{tabular} & & $\bullet$ & $\Delta$ & $\bullet$ & -1 & $\bullet$ & $\Delta$ & Q & - $\bullet$ & & 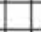 & $\boldsymbol{\Delta}$ & & & & - & S & & & & \\
\hline & $258 \mathrm{~A}-4-3,133-135$ & & & 0 & - & $\bullet$ & $\Delta$ & & & $\bullet$ & $\Delta$ & -4 & $\Delta \bullet$ & $\Delta$ & 口e & - $\mathbf{A}$ & & 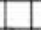 & $\Delta$ & & & & $\Delta$ & & & & & \\
\hline & $258 A-4-4,15-17$ & & & 0 & $\bullet$ & $\bullet$ & $\Delta$ & & & $\bullet$ & $\Delta$ & 4 & A & $\Delta$ & 口 & - $\Delta$ & & $\Delta$ & $\Delta$ & - & & & $\bullet$ & - & & & & \\
\hline & $258 \mathrm{~A}-4-4,45-47$ & & & 0 & $\bullet$ & $\bullet$ & - & & 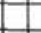 & - & $\Delta$ & 4 & $\Delta-$ & & 国 & \begin{tabular}{|l|l}
$\Delta$ & $\Delta$ \\
\end{tabular} & & $\Delta$ & $\Delta$ & & & & - & - & & & & \\
\hline & $258 \mathrm{~A}-4-4,75-77$ & & & 0 & $\bullet$ & $\Delta$ & $\Delta$ & & $\Delta$ & $\Delta$ & $\Delta$ & 6 & - $\Delta$ & $\Delta$ & 口 & - & & - & $\bullet$ & & & & -1 & - & & & & \\
\hline & $258 \mathrm{~A}-4-4,105-107$ & & & 0 & - & $\Delta$ & $\Delta$ & & & - & $\Delta$ & 9 & - $\mathbf{\Delta}$ & $\Delta$ & 口4 & $\Delta \mathbf{\Delta}$ & & & $\Delta$ & & & & 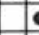 & $\bullet$ & & & & \\
\hline & $258 \mathrm{~A}-4-4,133-135$ & & & 0 & $\bullet$ & $\Delta$ & - & & - & $\bullet$ & $\Delta$ & 6 & - & $\bullet$ & 口e & - $\bullet$ & & & $\Delta$ & & & & 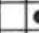 & - & & & & \\
\hline & $258 A-4-5,15-17$ & & & 0 & $\Delta$ & $\Delta$ & $\Delta$ & & $\bullet$ & - & $\Delta$ & - & $\mathbf{A}$ & $\Delta$ & 口 & $\Delta \mathbf{\Delta}$ & & & & & & & - & - & & & & \\
\hline & $258 A-4-5,45-47$ & & & 0 & $\bullet$ & $\bullet$ & $\Delta \bullet$ & $\Delta$ & - & - & $\Delta$ & 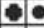 & - $\Delta$ & $\Delta$ & 口。 & - $\mathbf{\Delta}$ & & & & & & & - & - & & & & \\
\hline & $2589-4-5,75-77$ & & & 0 & $\bullet$ & $\Delta$ & \begin{tabular}{|l|l}
$\mathbf{A}$ & $\mathbf{A}$ \\
\end{tabular} & $\Delta$ & $\Delta$ & $\bullet$ & $\bullet$ & 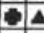 & $\Delta \Delta$ & & 口6 & $\bullet \bullet$ & & & & 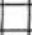 & 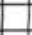 & & - & - & & & & \\
\hline & $258 \mathrm{~A}-4-5,105-107$ & & & 0 & $\Delta$ & $\bullet$ & $\Delta$ & & & $\bullet$ & $\bullet$ & 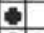 & $\Delta$ & - & $\square$ & e $\mathbf{A}$ & & & & $\Delta$ & & $\Delta$ & - & & & & & \\
\hline $45.5-$ & $258 A-4-5,133-135$ & & & 0 & $\Delta$ & $\bullet$ & $\Delta \bullet$ & & & - & $\bullet$ & 90 & $\boldsymbol{\Delta}$ & $\bullet$ & $\square$ & $\mathbf{A} \mathbf{A}$ & & E & 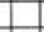 & $\Delta$ & $\Delta$ & & - & - & & & & \\
\hline $45.5-$ & $258 A-4-6,10-150$ & & & 0 & $\Delta$ & & & & $\Delta$ & $\bullet$ & $\bullet$ & & $\Delta$ & & $\square$ & - & & 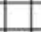 & $\mathbf{A}$ & & $\Delta$ & $\Delta$ & -1 & $\bullet$ & $\Delta \mid$ & $\Delta$ & & \\
\hline & $258 \mathrm{~A}-4, \mathrm{CC}$ & & & 0 & $\Delta$ & - & e & $\mathbf{A}$ & & - & $\Delta$ & - & $\bullet$ & & 口e & - $\bullet$ & & $\Delta$ & & & & & - & - & & & & \\
\hline $47.5-57.0$ & $258 \mathrm{~A}-5-1,30-32$ & & & 0 & $\Delta$ & $\bullet$ & e & $\bullet$ & & - & 6 & - & - & & 口e & - & & & & & 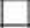 & & 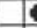 & 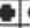 & - & $\Delta$ & & \\
\hline & $258-3-1,40-42$ & & & 0 & $\Delta$ & $\bullet$ & - & - & & $\bullet$ & - & 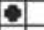 & - & & $\square$ & $\Delta$ & & & & & 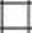 & & 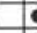 & $\bullet$ & $\Delta$ & & - & \\
\hline & $258 A-5-1,60-62$ & & & 0 & $\mathbf{\Delta}$ & $\Delta$ & e & $\Delta$ & $\Delta$ & - & 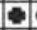 & - & $\bullet$ & & $\square$ & e & & & & & & & & 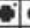 & 는 & $\bullet$ & - & \\
\hline & $2584-5-1,90-92$ & & & 0 & & $\bullet$ & - & $\Delta$ & & $\bullet$ & 6 & 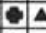 & $\mathbf{\Delta} \mathbf{\Delta}$ & & $\square$ & $\Delta$ & & & 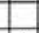 & & $\Delta$ & & & 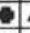 & $\Delta$ & $\Delta$ & - & \\
\hline & $258-3-1,110-112$ & & & 0 & $\Delta$ & $\bullet$ & - & $\Delta$ & & $\bullet$ & $\bullet$ & - & $\Delta$ & & - 1 & $\mathbf{A} \mathbf{\Delta}$ & & & $\mathbf{A}$ & - & & & & - & & & $\bullet$ & \\
\hline & $258 A-5-1,120-122$ & & & 0 & $\Delta$ & - & • & - & & - & $\mathbf{A}$ & $\bullet$ & $\Delta$ & & - & $\Delta$ & & & & & & & & - & & & $\Delta$ & \\
\hline & $258 A-5-1,148-150$ & & & 0 & $\mathbf{\Delta}$ & - & e & $\Delta$ & & - & $\bullet$ & 5 & $\mathbf{\Delta}$ & & 64 & $\mathbf{A} \mathbf{\Delta}$ & & & & & & $\Delta$ & & - & - & 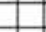 & $\bullet$ & \\
\hline & $258 \mathrm{~A}-5-2,30-32$ & & & 0 & $\bullet$ & $\bullet$ & 6 & $\Delta$ & & - & 6 & 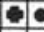 & - $\bullet$ & & $\square$ & • & & & & $\bullet$ & $\Delta$ & & & $\bullet$ & e & & • & \\
\hline & $258-3-2,40-42$ & & & 0 & $\bullet$ & $\bullet$ & e & & & $\Delta$ & $\bullet$ & - & $\mathbf{A}$ & & 回 & & & & & 6 & & & & 6 & & $\Delta$ & $\bullet$ & \\
\hline & $258 A-5-2,40-42$ & & & 0 & - & $\bullet$ & 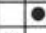 & & & $\Delta$ & - & - & $\Delta$ & & 畐 & & & & & 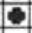 & & 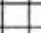 & 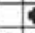 & 달 & & $\Delta$ & - & \\
\hline & $258 A-5-2,60-62$ & & & 0 & $\Delta$ & $\bullet$ & $\Delta$ & & & $\bullet$ & - & e & $\bullet$ & & $\square \Delta$ & $\Delta \Delta$ & & & & & & & - & - & 단 & & & \\
\hline & $2584-5-2,84-86$ & & & 0 & $\Delta$ & $\bullet$ & $\Delta$ & $\Delta$ & & $\Delta$ & 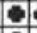 & 64 & $\Delta \mathbf{A}$ & & $\square$ & $\Delta$ & & & & $\Delta$ & & & $\Delta$ & $\Delta$ & $\Delta$ & & - & \\
\hline & $258-3-2,110-112$ & & & 0 & & -1 & $\bullet \bullet$ & $\Delta$ & & $\bullet$ & - & - & $\bullet$ & & - & $\Delta$ & & & & & $\Delta$ & & & • & & $\Delta$ & - & \\
\hline & $258 \mathrm{~A}-5-2,120-122$ & & & 0 & & $\bullet$ & $\Delta \bullet$ & $\mathbf{A}$ & & & & $\bullet$ & $\bullet$ & & - & $\Delta$ & & & & & & & $\Delta$ & $\Delta$ & $\Delta$ & $\mathbf{A}$ & & \\
\hline & $258 A-5-2,147-149$ & & & 0 & $\Delta$ & $\bullet$ & $\Delta$ & $\bullet$ & & $\Delta$ & 口 & $\bullet$ & $\Delta$ & & $\varphi$ & & & & & & & & & & 4 & \begin{tabular}{c|c} 
\\
\end{tabular} & & \\
\hline & $258 \mathrm{~A}-5-3,30-32$ & & & 0 & $\Delta$ & $\Delta$ & $\Delta$ & & & $\Delta$ & T & $\bullet$ & & & - & $\bullet$ & & & & & & & 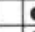 & e & 4 & $\mathbf{\Delta} \mathbf{\Delta}$ & & \\
\hline & $258-3-3,40-42$ & & & 0 & $\Delta$ & & - $\Delta$ & & & $\Delta$ & - & $\bullet$ & $\bullet$ & & 4 & \begin{tabular}{|c|}
$\mathbf{A}$ \\
\end{tabular} & & & $\Delta$ & & & & & 5 & & & - & \\
\hline & $258 \mathrm{~A}-5-3,40-42$ & & & 0 & $\Delta$ & & - $\Delta$ & & & $\mathbf{\Delta}$ & $\bullet$ & - & $\bullet$ & & 64 & \begin{tabular}{|c|c} 
\\
\end{tabular} & & & $\Delta$ & & & & 5 & 5 & & $\square$ & - & \\
\hline & $258 \mathrm{~A}-5-3,60-62$ & & $\ddot{\Sigma}$ & 0 & $\bullet$ & $\Delta$ & $\Delta$ & $\Delta$ & & & - & -4 & $\mathbf{A} \mathbf{A}$ & & $\mathbf{D}$ & $\Delta \bullet$ & & $\Delta$ & & 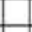 & & & 4 & 5 & & $\Delta$ & $\Delta$ & \\
\hline & $258 \mathrm{~A}-5-3,90-92$ & & $\stackrel{8}{\circ}$ & 0 & $\bullet$ & $\Delta$ & e & $\mathbf{A}$ & $\Delta$ & $\bullet$ & 6 & 24 & $\boldsymbol{\Delta} \mathbf{\Delta}$ & & $\mathbf{a}$ & A. $\mathbf{A}$ & & & & & & & 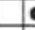 & - & $\Delta$ & $\Delta$ & $\bullet$ & $\Delta$ \\
\hline & $258-3-3,115-117$ & జี & $\bar{a}$ & 0 & $\Delta$ & - & & $\Delta$ & & - & - & & $\Delta$ & & a & $\mathbf{A}$ & & & & $\Delta$ & & & 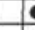 & •. & $\Delta$ & $\Delta$ & & \\
\hline & $258 A-5-3,120-122$ & & $\div$ & 0 & $\Delta$ & $\Delta$ & & $\Delta$ & & - & & D & | & & \begin{tabular}{|l|l} 
\\
\end{tabular} & $\Delta \mathbf{A}$ & & & & $\Delta$ & & & 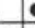 & • & $\Delta$ & D & $\Delta$ & \\
\hline & $258 A-5-3,147-149$ & & 물 & 0 & & $\bullet$ & & $\Delta$ & & $\bullet$ & & - & 1 & & 4 & $\Delta \mid \Delta$ & & & & & & & $\Delta$ & • & - & $\Delta$ & & \\
\hline & $2584-5-4,30-32$ & & & 0 & & - & $\bullet$ & $\Delta$ & & - & $\bullet$ & $\bullet 4$ & $\Delta \bullet$ & & 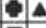 & $\Delta$ & & & & $\Delta$ & & - & $\Delta$ & • & $\Delta$ & $\Delta$ & $\Delta$ & \\
\hline & $258-3-4,40-42$ & & & 0 & & -1 & - & $\bullet$ & & & - & - & - & & ( & & & & & - & & & 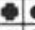 & •] & $\Delta$ & $\Delta$ & & \\
\hline & $258 A-5-4,60-62$ & & & 0 & & $\Delta$ & - $\bullet$ & $\bullet$ & & & - & $\bullet$ & & & [e & - & & & & $\Delta$ & & & - & - & $\Delta$ & & & \\
\hline & $258 A-5-4,90-92$ & & & 0 & & - & $\Delta \bullet$ & $\Delta$ & & - & 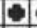 & - & $\Delta$ & & [0 & & & & & & & & $\Delta$ & 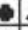 & $\Delta$ & & & \\
\hline & $258-3-4,115-117$ & & & 0 & $\Delta$ & $\Delta$ & $\Delta \Delta$ & & & - & -1 & $\bullet$ & $\Delta$ & & a & & & & & $\Delta$ & & - & - & 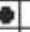 & & & & \\
\hline & $258 \mathrm{~A}-5-4,120-122$ & & & 0 & $\Delta$ & $\Delta \mid$ & $\Delta$ & & & $\bullet$ & 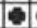 & $\bullet$ & & & a & $\Delta$ & & & & & & & & & $\Delta$ & & & \\
\hline & $258 \mathrm{~A}-5-4,147-149$ & & & 0 & $\bullet$ & $\bullet$ & 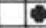 & & & $\Delta$ & 6 & & $\bullet$ & & DA & \begin{tabular}{|l|l|}
$\mathbf{A}$ & \\
\end{tabular} & & & & & & & - & 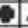 & $\Delta$ & & & \\
\hline & $258 A-5-5,15-17$ & & & 0 & & $\bullet$ & - & $\Delta$ & & $\bullet$ & 한 & 0.4 & $\Delta \bullet$ & & 5 & & & & & & & & -1 & 6 & $\boldsymbol{\Delta}$ & & & \\
\hline & $258-3-5,40-42$ & & & 0 & & $\bullet$ & e & $\bullet$ & & - & $\varphi$ & 14 & $\Delta \bullet$ & & 8 & $\Delta$ & & & & & & & & 6 & $\Delta$ & $\Delta$ & & - \\
\hline & $258 A-5-5,45-47$ & & & 0 & & $\bullet$ & e & $\Delta$ & & e & 6 & e & $\Delta$ & & $\bullet$ & & & & & & & & & & $\Delta$ & - & $\Delta$ & \\
\hline & $258 A-5-5,75-77$ & & & 0 & & - & - & $\bullet$ & & - & 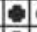 & $\bullet$ & $\bullet$ & & $\div 0$ & e & & & & & & & - & 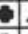 & $\Delta$ & $\Delta$ & $\Delta$ & \\
\hline & $258 \mathrm{~A}-5-5,105-107$ & & & 0 & & $\bullet$ & - & $\bullet$ & & $\bullet$ & - & $\bullet$ & $\bullet$ & & 量 & & & & & & & & -1 & 6 & $\Delta$ & $\Delta$ & & \\
\hline & $258-3-5,136-138$ & & & 0 & - & $\Delta$ & 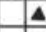 & $\Delta$ & & $\Delta$ & $\bullet$ & $\bullet$ & $\Delta$ & & 国 & & & & & & & $\mathbf{A}$ & 1 & •. & $\Delta$ & - & & - \\
\hline & $2584-5-5,138-140$ & & & 0 & - & $\Delta$ & - & $\Delta$ & & $\Delta$ & $\bullet$ & $\bullet$ & $\Delta$ & & 国 & & & & & & $\Delta$ & - & 5 & e. & $\Delta$ & - & & - \\
\hline & $258 A-5-6,15-17$ & & & 0 & $\bullet$ & & - & $\Delta$ & & & 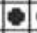 & $\bullet$ & $\Delta$ & & - & & & & & & & $\Delta$ & $\Delta$ & •. & $\Delta$ & $\Delta$ & & - \\
\hline & $258-3-6,40-42$ & & & 0 & & & - & $\Delta$ & & $\Delta$ & 6 & $\bullet$ & - & & 国 & & & & & & & & & & $\Delta$ & $\bullet$ & & 6 \\
\hline & $258 A-5-6,45-47$ & & & 0 & $\Delta$ & $\bullet$ & - & & & $\bullet$ & 6 & & $\Delta$ & & - 4 & $\Delta \mathbf{\Delta}$ & & & & & & $\Delta$ & - & $\bullet$ & $\Delta$ & $\Delta$ & & 달 \\
\hline & $258 \mathrm{~A}-5-6,75-77$ & & & 0 & $\Delta$ & $\bullet$ & e & & & $\bullet$ & 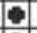 & & $\Delta$ & & - & $\Delta$ & & & & & & $\Delta$ & $\Delta$ & . & $\Delta$ & $\Delta$ & & - \\
\hline & $258 \mathrm{~A}-5-6,105-107$ & & & 0 & $\Delta$ & $\bullet$ & $\bullet$ & & & $\bullet$ & $\bullet$ & & $\Delta$ & & - & & & & & & & $\Delta$ & & & $\Delta$ & & & - \\
\hline & $258-3-6,115-117$ & & & 0 & & $\bullet$ & $\Delta \bullet$ & & & & $\square$ & $\bullet$ & $\bullet$ & & - & & & & & & $\Delta$ & & & $\Delta$ & $\Delta$ & & & - \\
\hline & $258 \mathrm{~A}-5-6,135-140$ & & & 0 & - & $\bullet$ & $\Delta$ & $\Delta$ & & $\Delta$ & - & 014 & $\Delta \bullet$ & & 国 & & & & & & $\Delta$ & $\bullet$ & & - & 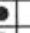 & $\Delta$ & & $\Delta$ \\
\hline & $258-3, c C$ & & & 0 & - & $\Delta$ & $\mathbf{\Delta}$ & $\mathbf{A}$ & & $\Delta$ & 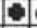 & 04 & $\Delta \bullet$ & & a & & & $\Delta$ & & & & - & 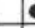 & •- & - & $\Delta$ & & r \\
\hline & $258 \mathrm{~A}-5, \mathrm{CC}$ & & & 0 & - & $\Delta$ & $\Delta$ & $\Delta$ & & $\Delta$ & & $\bullet 4$ & $\Delta \bullet$ & & a & & & $\Delta$ & & & & $\bullet$ & & & e 1 & $\mathbf{A}$ & & \\
\hline
\end{tabular}

Figure 7. (Continued). 


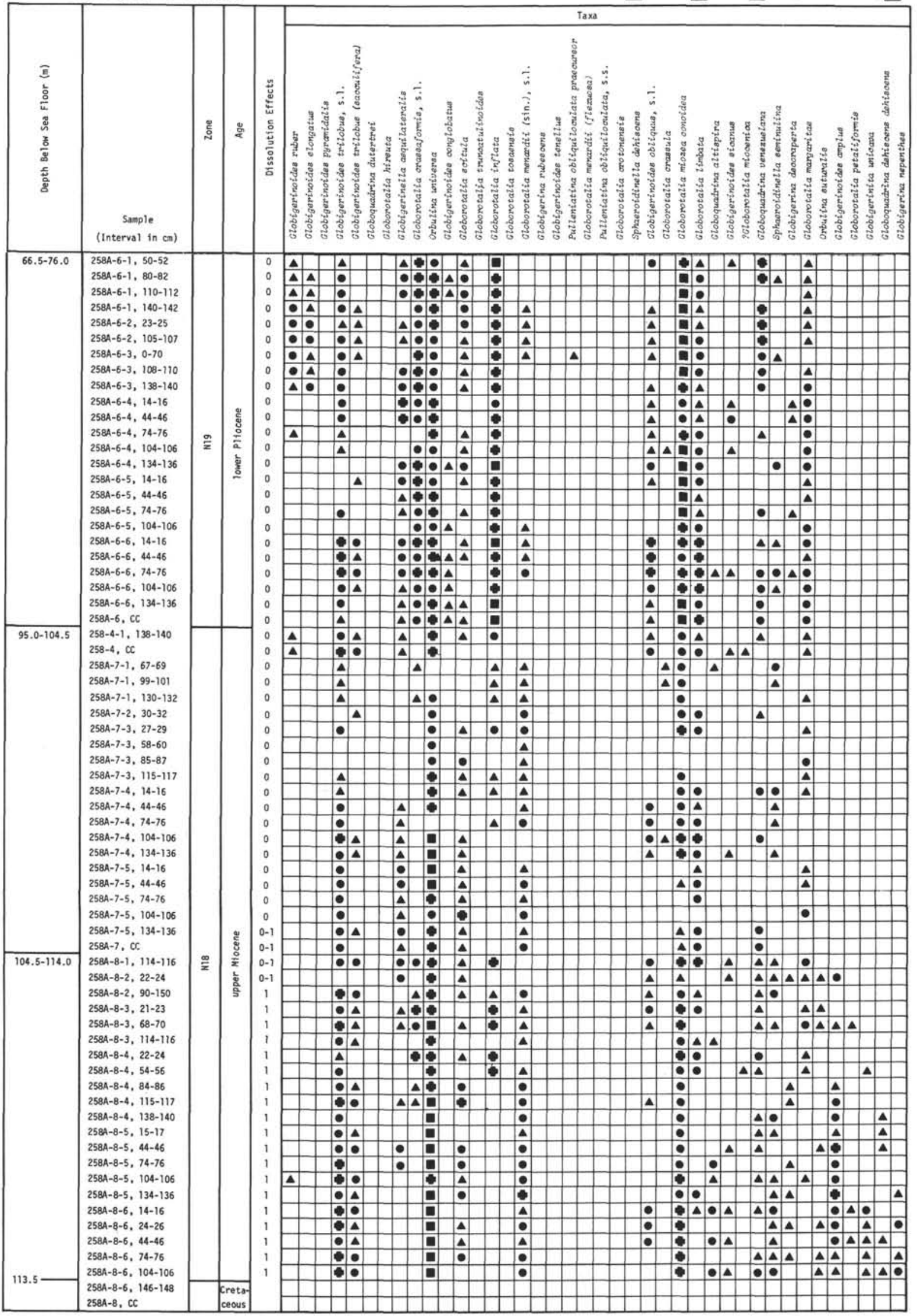

Figure 7. (Continued). 
It is interesting to mention that, as at Sites 253 and 254 , the Miocene assemblages at Site 258 contained a great number of very small unidentifiable planktonic foraminifera.

The upper Miocene sediments were the oldest Neogene deposits encountered at Site 258. At the depth of 114 meters, Cretaceous deposits were found.

Quaternary foraminiferal assemblages of Site 258 are typical of the temperate zone. Globorotalia inflata, s.l. strongly predominates and G. menardii, s.l. is recorded sporadically and as isolated individuals.

Site 258 showed a rather enigmatic stratigraphic range for Globigerinoides sicanus. For detail see the Systematics Section, below.

\section{CORRELATION OF SITES AND SEDIMENTATION RATES}

Sites $250,251,254,255$, and 258 were selected for correlation of their stratigraphical columns. These sites are located latitudinally between the parallels $31^{\circ}$ and $36^{\circ} 30^{\prime} \mathrm{S}$. The Miocene/Pliocene and Pliocene/Quaternary boundaries were correlated; the zones, however, were not correlated. In cases where these boundaries could not be located precisely, they were placed in the middle of the indefinite section and marked with a dashed line (Figure 8). Lithologic character is noted in a very summarized and abbreviated form. For lithological details see the special paper on lithology in this volume.

The resulting correlation chart (Figure 8) shows clearly that the minimum thickness of Quaternary, Pliocene, and Miocene deposits is at Sites 254 and 255. These sites are located on Ninetyeast Ridge and Broken Ridge, respectively, and for this reason they have the shallowest depths. On both sides of these ridges the thickness of the Neogene deposits increases. It is especially great on the western side near the African continent where, at Site 250 , sediments are composed chiefly of terrigenous material (detrital clay).

The different thicknesses are the result of different rates of accumulation. Near the continent, accumulation is the most rapid because of terrigenous material brought from the land. Depth is a factor because in deep areas calcareous material is dissolved. Bottom relief also plays a role; it has been established that on submarine rises the sedimentation rate is much lower than in basins. The correlation chart presented is a good example of the influence of all these factors.

For the determination of sedimentation rates, age dates used were taken from Berggren's study (Berggren, 1972). According to Berggren, the Quaternary lasted 1.8 m.y., the Pliocene 3.2 m.y., and the Miocene 17.5 m.y. The consolidation of the sediments was not taken into account in calculating the sedimentation rates. The following values were obtained.

1) For the Quaternary: between $3 \mathrm{~m} / \mathrm{m} . \mathrm{y}$. (Site 254) and approximately $54 \mathrm{~m} / \mathrm{m} . \mathrm{y}$. (Site 250 ).

2) For the Pliocene: between $4 \mathrm{~m} / \mathrm{m} . \mathrm{y}$. (Site 255) and approximately $31 \mathrm{~m} / \mathrm{m} . \mathrm{y}$. (Site 250 ).
3) For the Miocene: between approximately 1.4 $\mathrm{m} / \mathrm{m} . \mathrm{y}$. (Site 255 ) and approximately $27 \mathrm{~m} / \mathrm{m} . \mathrm{y}$. (Site 250).

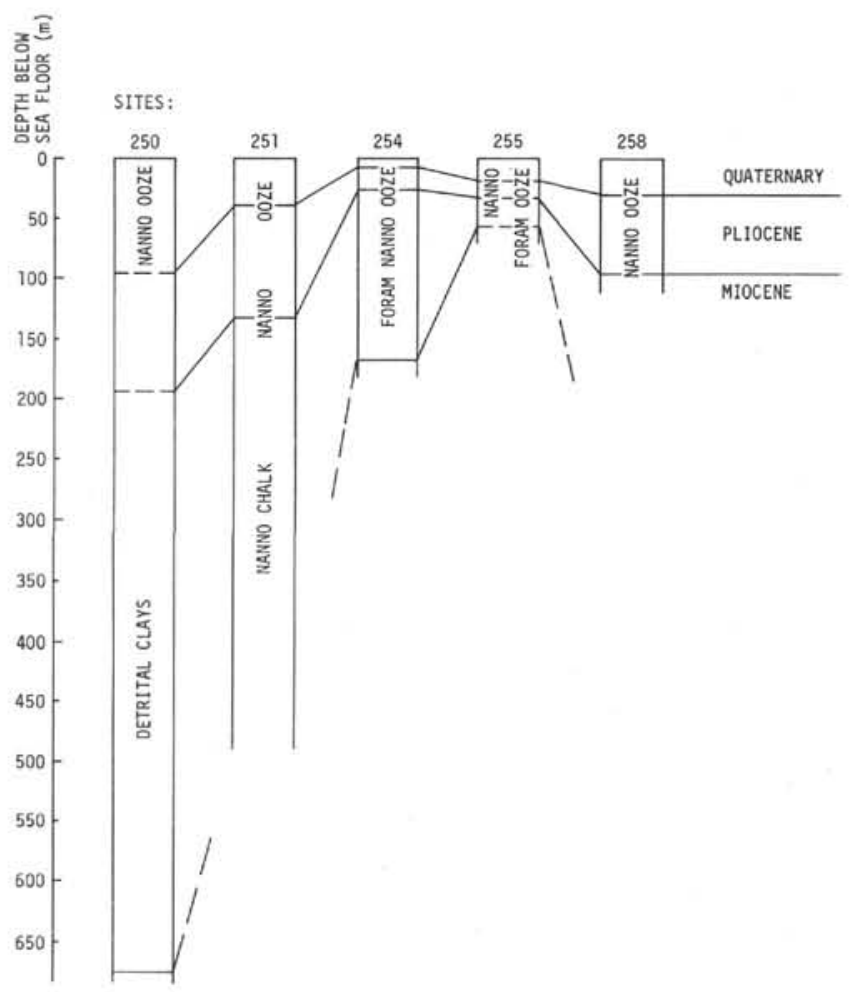

Figure 8. Correlation of the sites.

\section{GENERAL RANGE CHART OF SELECTED SPECIES}

Records of the vertical distribution of the 70 selected foraminiferal planktonic species at all the sites were taken into consideration to prepare a general biostratigraphical range chart (Figure 9).

It should be emphasized that this range chart is based only on the data obtained from this study and is not influenced by studies carried out by other investigators. Globigerinita unicava, for instance, is well known from Oligocene. Nevertheless, because in the present material its oldest occurrence was in the early Miocene, I put its range beginning at that age. It is quite probable that if I could have studied more Oligocene material, I could have extended its range.

The ranges of the majority of the species were found to be approximately the same as those established by authors who studied other areas. There are some small differences which are not important. The same species does not necessarily appear at the same time in all areas. However, the biostratigraphic ranges of several species appear to be considerably different from ranges known 


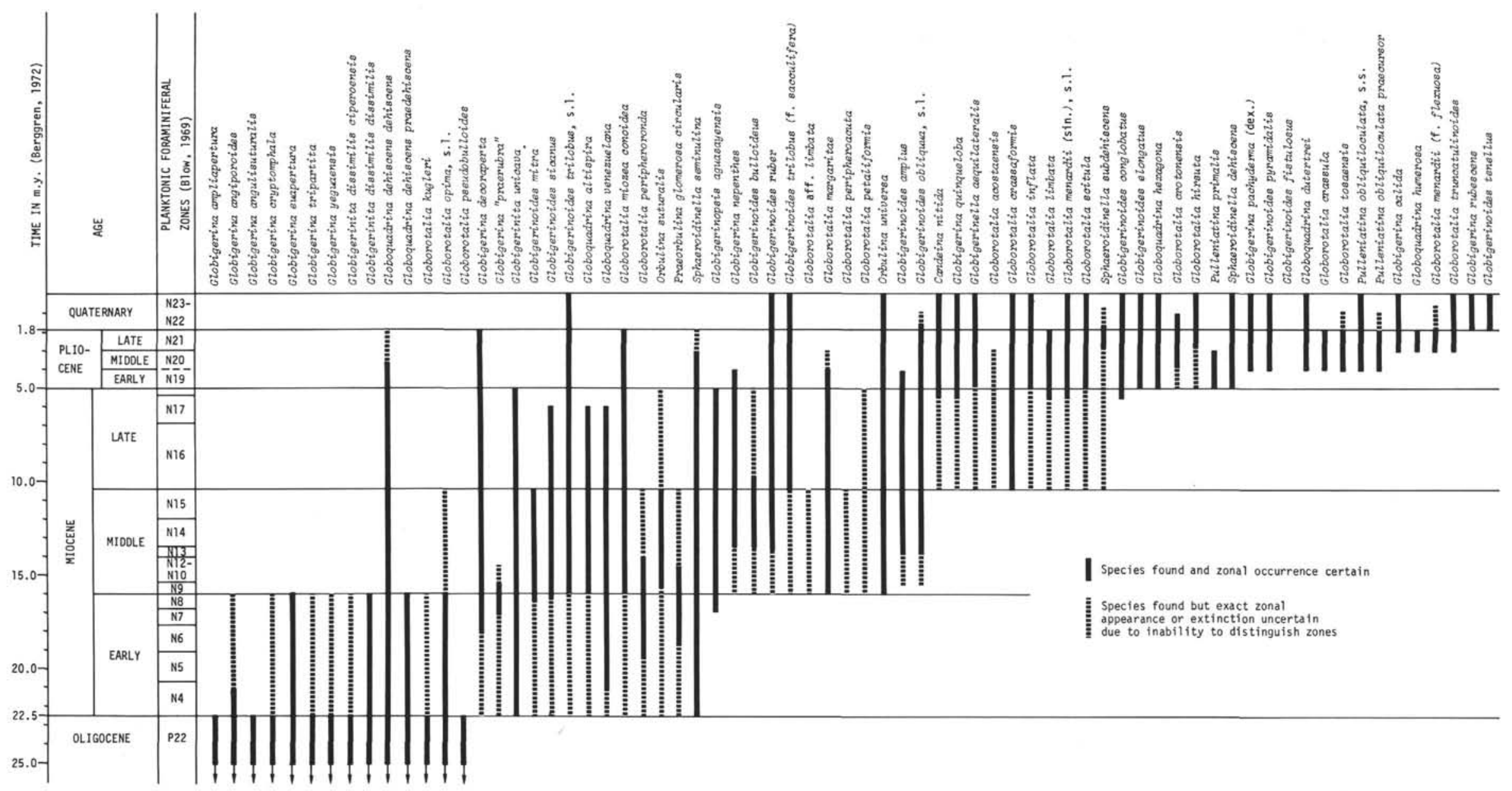

Figure 9. General range chart of selected species. 
from the literature. Some of them were found in older layers, but as the lower limit of this study is the Miocene/Oligocene boundary, as might be expected, more often they were found in deposits younger than those recorded by previous authors. Since some of these species are used by micropaleontologists as marker species, I found it of interest to prepare the following table for these species (Table 2).

The differences listed above are substantial and need explanation. The following three explanations can be suggested.

1) Determinations of the ages of the Leg 26 samples were wrong. I do not think that such is the case, because age identification was checked using other foraminiferal assemblages and nannoplankton data.

2) Reworked specimens of the species cited were found in the younger deposits. This explanation should be denied too, not only because of the state of preservation of the specimens under discussion (this reason can be sometimes misleading), but also for the following reasons. If we admit that they are reworked, how can we explain that in many cases specimens of only one presumably reworked species are found in the in situ assemblage, and no specimens of other species, which are much more resistant to dissolution? For instance, at Site 258 many specimens of Globigerinoides sicanus were found in the Pliocene material, but none of the other foraminifera which are also considered as typical Miocene markers.

3) The third explanation is that the species mentioned really had higher ranges (at least in the Indian Ocean) than have yet been accepted. I think that this explanation is the best one, because some of these species have already been found in deposits much younger than those recorded in the literature. Baumann (1972), for instance, found Globigerina cryptomphala, $G$. tripartita, and Globorotalia opima, s.l. in the early Miocene of Borneo. Soediono (1970) encountered in Spain, in the Espejos formation (which is considered to be early Miocene-middle Miocene in age), Globigerina tripartita and Globorotalia opima, s.l.

It is certainly possible that taxonomical identifications in this report are wrong. But, as I stated above, maximum attention was given to the correct identification. All the species cited in Table 2 are illustrated in the plates, thus identifications can be roughly checked.

\section{SYSTEMATIC PALEONTOLOGY}

This section contains a very brief discussion of the species considered as the most significant and representative of the Neogene deposits of the central part of the Indian Ocean.

Very few species have been excluded from the range charts and still fewer from the discussion.

For the convenience of the readers, all species are arranged in alphabetical order. Neither primary citations nor synonymies are given for the species cited. However, scanning electron photographs of almost all the species discussed are presented, and at the end of this report a faunal reference list is included.

I am aware that several of the species listed probably do not have real zoological value and are junior synonyms of previously established taxa. I am also of the opinion that in some cases the wrong

TABLE 2

The Youngest Recorded Age of Extinction

\begin{tabular}{lll}
\hline \multicolumn{1}{c}{ Species Name } & \multicolumn{1}{c}{ According to Literature } & According to This Report \\
\hline Globigerina angiporoides & early Oligocene (Blow, 1969) & early Miocene \\
Globigerina cryptomphala & late Eocene (Glaessner, 1937) & lower Miocene \\
Globigerina tripartita & late Oligocene (Blow, 1969) & lower Miocene \\
Globigerina yeguaensis & late Oligocene (Postuma, 1971) & early Miocene \\
Globigerinita unicava & early Miocene (Blow, 1969) & late Miocene \\
Globigerinoides sicanus & middle Miocene (Postuma, 1971) & middle Pliocene \\
Globorotalia miozea conoidea & late Miocene (Jenkins, 1967) & late Pliocene \\
Globorotalia pseudobullodes & Eocene (Plummer, 1926) & Oligocene \\
Globorotalia opima, s.l. & late Oligocene (Blow, 1969) & middle Miocene \\
\end{tabular}


subspecific name was used. I cited, for the same sample for instance, two subspecific names of the same species. However, two planktonic subspecies can be encountered in the same sediment sample only if during their life they occupied quite different depth ranges, and this is not very probable. Not having sufficient material and time for real taxonomic studies, 1 preferred, in these cases, not to enter into the taxonomical details and leave existing taxa such as they are in common stratigraphic usage.

The present report is not primarily a taxonomic work. Its main aim is the examination of age determinations and biostratigraphy, and where possible, the drawing of paleoclimatological conclusions. Nevertheless, in some cases, which were evident and did not require special detailed taxonomic studies, I have drawn some taxonomic conclusions and even established a new species. In addition, I have used some taxonomic conclusions regarding living species which were the result of previous studies of Recent faunas, but which were sometimes not in agreement with common usage by micropaleontologists.

Where the statement "found at all the sites" was used, it should be understood to mean only those sites which contained preserved fauna. Sites 252, 256, and 257, because of their great depth, were barren.

All hypotypes are deposited in the Foraminiferal Collection of Museo Argentino de Ciencias Natureales "B. Rivadavia" (FMACN) and have corresponding numbers.

\section{Candeina nitida d'Orbigny, 1839 \\ (Plate 1, Figure 4)}

This foraminifer is a typical warm-water species. It was found only at the northernmost site (Site 253) and was represented by rather large but never common specimens. At Site 253 it appeared in the upper Miocene and was recorded throughout the section, with some short interruptions, up to the top sample.

\section{Globigerina ampliapertura Bolli, 1957}

(Plate 1, Figures 1-3, 5, 6)

According to Bolli (1966) and Blow (1969), this species ranges from the upper Eocene into the Oligocene. It was found only in the Oligocene deposits at Site 253 and was represented by good typical specimens. Rare specimens encountered in the lower Miocene of Site 251 were apparently redeposited.

\section{Globigerina angiporoides Hornibrook, 1965}

(Plate 1, Figures 7, 8)

Rare but very typical representatives of this species were found in the Oligocene section at Sites 253 and 254. In the latter site some isolated specimens were recorded from the lower Miocene sediments indicating that perhaps it would be right to extend the stratigraphic range of this species. Specimens found did not look reworked.

\section{Globigerina angulisuturalis Bolli, 1957}

Very rare specimens of this species were found in Sample 20, CC of Site 254 (Oligocene).

\section{Globigerina bulloides d'Orbigny, 1826 (Plate 2, Figure 4)}

As explained above, G. bulloides and several closely related species are not included in the range charts. However, it should be noted that when considering paleoclimatic conditions, the $G$. bulloides group was taken into account as typical representatives of cold-temperate waters.

$G$. bulloides-group representatives were found rather frequently at Sites 254 and 258. They were less frequent at Site 251 and infrequent at Sites 253 and 250 . At the last site the small number of $G$. bulloides is probably explained by the effects of dissolution.

Figure 4, Plate 2 shows a typical representative of $G$. bulloides.

\section{Globigerina calida Parker, 1962} (Plate 1, Figure 9)

G. calida is a foraminifer which connects G. bulloides and Globigerinella aequilateralis. It is more characteristic and more easily distinguishable from $G$. bulloides than $G$. falconensis. In the material studied typical specimens were found very rarely in the upper Pliocene-Quaternary section of Sites 250 and 251.
Globigerina cryptomphala Glaessner, 1937

(Plate 2, Figures 1, 2, 5-10)

This species was found in the Oligocene and lower Miocene deposits at Sites 250,253, 254, and 255. At Site 251 G. cryptomphala was not present, probably because of the southern location of this site. This species evidently preferred the warm waters of low latitudes. Glaessner interpreted this foraminifer as an abnormal form of Globigerina bulloides and gave a rather schematic species description. However, he emphasized that it is very abundant in the upper Eocene deposits of the North Caucasus and does not appear later. The most characteristic feature of this species, according to Glaessner, is a small, poorly developed last chamber which partially covers the umbilical aperture. The final whorl has four normal chambers.

Among several hundred specimens found, many looked exactly like the holotype figured by Glaessner. However, many specimens were not quite identical. The main difference was in the shape and location of the final, poorly developed chamber. As a matter of fact it is not a real chamber but rather a patch or valve whose main purpose is most probably to protect the entrance of the aperture.

Figures 1, 2, 5, 7, and 9 on Plate 2 show typical representatives of $G$. cryptomphala. Figures 6,8 , and 10 on the same plate show different kinds of aberrations in the form and location of the protecting valve. Some specimens were found without any protecting valve.

The range of $G$. cryptomphala found in the Leg 26 material is considerably younger than that given by Glaessner. For details see the range charts in Figures 2-7.

\section{Globigerina decoraperta Takayanagi and Saito, 1962}

(Plate 1, Figure 10)

$G$. decoraperta was represented at all the sites by rather typical specimens. It ranged from the lower Miocene into the upper Pliocene. Unfortunately, this species grades in its upper range into $G$. rubescens and in its lower range into $G$. woodi. In addition, it is very closely related to $G$. druryi and to a certain degree to $G$. apertura. This circumstance creates difficulties in identifying it correctly and thus decreases the value of $G$. decoraperta as a stratigraphic indicator.

\section{Globigerina euapertura Jenkins, 1960}

(Plate 1, Figures 11, 12)

This foraminifer occurred at three sites, principally the northern ones, indicating it is a warm-water species. It possesses a tightly coiled test and a low-arched, sometimes not very regular, shaped aperture. It ranges stratigraphically from the Oligocene through the lower Miocene.

\section{Globigerina falconensis Blow, 1959} (Plate 2, Figure 3)

This foraminifer undoubtedly is very closely related to G. bulloides, representing its subtropical "branch." It was not separated from the G. bulloides group.

\section{Globigerina juvenilis Bolli, 1957}

(Plate 1, Figure 13)

This species was found to be sporadic and rare in the Oligocene and lower Miocene section at Site 253.

\section{Globigerina linaperta Finlay, 1939 \\ (Plate 1, Figure 14)}

According to Finlay this a middle Eocene species. Blow (1969) considered it as late Eocene. Isolated but rather typical specimens of this species were found in Sample 19, CC at Site 250. They were well preserved and did not look reworked; however, the whole of Core 19 at Site 250 is lower Miocene (Globigerinoides trilobus, Globorotalia kugleri) in age. It is not clear whether the stratigraphic range of $G$. linaperta should be changed, or the specimens found are not in situ but are reworked.

\section{Globigerina nepenthes Todd, 1957}

(Plate 1, Figures 15, 16)

This very typical and easily identifiable species was found at almost all the sites studied, sometimes in great numbers. It ranges upward, 
according to Blow (1969), Kaneps (1973), and Parker (1973) through the lower Pliocene. Poag and Akers (1967) recorded it from the middle Pliocene, accompanied by Globorotalia truncatulinoides, $G$. inflata, and Sphaeroidinella dehiscens. At Site $255 \mathrm{G}$. nepenthes was found with the same species; however, I am inclined to consider the sediments (because of the occurrence of $G$. truncatulinoides) as upper Pliocene. This is the highest appearance of the species discussed. In other sites $G$. nepenthes ranges only through the lower Pliocene.

\section{Globigerina pachyderma (Ehrenberg, 1873)}

(Plate 2, Figures 11, 12)

Only the dextral form of this species was found and only at the southernmost site (Site 251). This species, especially its sinistral specimens, is a typical cold-water indicator. Dextral-coiled tests characterize Subantarctic waters and can be found also in the temperate zone where mixing of Subantarctic waters takes place. $G$. pachyderma ranges from Pliocene to Recent. At Site 251 it was recorded from the section which was determined as lower and/or middle Pliocene in age.

\section{Globigerina "praerubra"}

(Plate 2, Figures 13-16)

This species may be new; I have not been able as yet to find any known species to which it could be ascribed. However, I prefer for the time being not to establish a new taxon on the basis of the available material. Therefore, temporarily, the species name is in quotation marks. It is a temporary name given only for use in this report.

At first glance G. "praerubra" looks rather like Globigerinoides ruber. It is low trochospiral and its umbilical side has the same pattern of three chambers and a rounded aperture in the final chamber which is located opposite the penultimate and antepenultimate chambers. However, it is different from $G$. ruber in several ways. First of all no supplementary apertures are present; thus the species discussed belongs not to the genus Globigerinoides but Globigerina. Also, in many specimens the aperture shows a clear tendency to be asymmetrical. In some cases almost four chambers are in the final whorl and thus the aperture is situated opposite the two-and-a-half or even three preceding chambers. These specimens are similar to those of the Globigerina decoraperta group.

G. "praerubra" is perhaps an ancestor of Globigerinoides ruber. The latter species ranges from middle Miocene to Recent. The same range of Globigerinoides ruber was observed in the present material. G. "praerubra" was recorded in the three northern sites (Sites 253-255) in the lower Miocene, with the exception of Site 254 where it was also found in the middle Miocene. Blow (1969) stated that the developmental trend of Globigerinoides trilobus is from a test with only one aperture to those with several. It is quite possible that the same trend took place in Globigerinoides ruber.

\section{Globigerina quinqueloba Natland, 1958}

$$
\text { (Plate 3, Figures 1, 2) }
$$

Typical specimens of this species were found only in the Quaternary and upper Miocene deposits of Site 250. According to Parker (1973), G. quinqueloba ranges at least from the upper Miocene. It is a coldand cool-water species; therefore, it is unlikely to be common in the material under study.

\section{Globigerina rubescens Hofker, 1956}

(Plate 4, Figure 2)

I found this species only in Quaternary deposits at Site 258. It is interesting to note that even in the lowermost sample which contained this species (depth $8.5 \mathrm{~m}$ below bottom surface) several tests were pink. Parker (1973) gives this species a range from the middle Pliocene and states that pink specimens are marker specimens for the Holocene.

\section{Globigerina tripartita Koch, 1926}

(Plate 3, Figures 5-10)

There is some confusion with respect to the interpretation of this species, as it has much similarity with Globigerina rohri and several other related species living more or less during the same epoch.
In this study I considered as G. tripartita those specimens which had three chambers in the final whorl, did not possess apertural teeth, and whose spiral side was convex. A peculiar feature, which many specimens in the present material had, was a small poorly developed bullalike chamber, which looked similar to the protecting valve described in G. cryptomphala, and partially covered the umbilical aperture. This formation was previously observed by Soediono (1970) who studied foraminifera of the Espejo formation (early Miocene to middle Miocene) in Spain and found a very high number of specimens of $G$. tripartita with the same anomalous final chamber.

Sometimes it was rather difficult to separate G. tripartita from Globoquadrina dehiscens praedehiscens. However, in their typical representatives they do differ, as $G$. dehiscens praedehiscens has apertural teeth, its dorsal side is less convex (almost flat), and its peripheral outline is somewhat angular.

According to Blow (1969), G. tripartita ranges from upper Eocene to upper Oligocene. I found this species in the two northern sites (Sites 253 and 254), and in both cases it ranged from Oligocene to lower Miocene. This was discussed previously.

\section{Globigerina yeguaensis Weinzierl and Applin, 1929} (Plate 3, Figures 11, 12)

Rather typical representatives of this species were found at the three northern sites (Sites 253-255). They ranged from Oligocene to lower Miocene inclusively.

\section{Globigerinella aequilateralis (Brady, 1884) \\ (Plate 3, Figure 3)}

Many typical specimens were found at all the sites except the two northern ones (Sites 253,254). This indicates that the species prefers warm-temperate waters but not warm water. The oldest sediments where $G$. aequilateralis was encountered are upper Miocene in age.

\section{Globigerinita cf. africana Blow and Banner, 1962}

(Plate 3, Figures 13-16)

The most characteristic features of this species, according to Blow and Banner, are its very large bulla which covers the umbilicus and three accessory apertures (at least) which are easily distinguishable. My specimens have the same type of bulla and the other morphological characteristics are also very similar to those of $G$. africana with the exception of the supplementary apertures. These are very badly developed in specimens from the Leg 26 material; sometimes it was practically impossible to find them. This circumstance forced me to use the designation "cf."

As for the range of $G$. africana, it is, according to Blow (1969), from P14 to P16 Zones (upper Eocene). G. cf. africana was found at Sites 253-255 in Oligocene and lower Miocene deposits.

\section{Globigerinita dissimilis ciperoensis Blow and Banner, 1962}

(Plate 4, Figures 1, 5)

This subspecies was separated from $G$. dissimilis dissimilis (nominat subspecies) by Blow and Banner chiefly on the basis of the difference in their bulla. Whereas the bulla in $G$. dissimilis dissimilis is attached to the final and antepenultimate chambers only, and thus produces two accessory apertures, in $G$. dissimilis ciperoensis a bulla produces three or even four accessory apertures.

This subspecies was found as isolated specimens in the Oligocene and lower Miocene sections.

\section{Globigerinita dissimilis dissimilis (Cushman and Bermúdez, 1937)} (Plate 4, Figure 6)

This subspecies is somewhat more frequent than the preceding one, but its range at the sites studied is similar to that of $G$. dissimilis ciperoensis.

\section{Globigerinita glutinata (Egger, 1893) (Plate 3, Figure 4)}

Rare specimens were found at Site 250 where their occurrence was sporadic from the upper Miocene to the Quaternary inclusively. 
Globigerinita unicava (Bolli, Loeblich, and Tappan, 1957) (Plate 4, Figures 7-9)

The most distinctive characteristic of this species is its bulla which is subquadrate and is attached at three sides leaving an arched infralaminal accessory opening on the fourth side. As in the other species with a formation partially covering the primary aperture, the bulla of $G$. unicava is rather changeable in shape and, to some degree, position.

Although, according to Blow (1969), this foraminifer ranges from Oligocene to lower Miocene, it was found at Site 254 in the middle Miocene and at Sites 253 and 258 even in the upper Miocene.

\section{Globigerinoides amplus Perconig, 1968}

(Plate 4, Figures 10-15)

This taxon was established by Perconig as a subspecies of Globigerinoides obliquus Bolli. However, specimens found in the present study (Sites 253 and 258) were so typical and easily distinguishable from $G$. obliquus that I preferred to raise this taxon to the specific rank. It should be mentioned that the specimens had much similarity with $G$. emeisi. Nevertheless, the presence of many supplementary apertures ( $G$. emeisi has only one) and in many cases a very high trochospiral test (G. emeisi is low trochospiral) in these specimens indicates that they are much closer to $G$. amplus.

G. amplus was recorded in the present material in the upper and also in middle Miocene. This extends downward the upper Miocene-lower Pliocene range of this foraminifer given by Perconig.

\section{Globigerinoides bulloideus Crescenti, 1966}

(Plate 4, Figures 3, 4)

This species was recorded from middle Miocene. Parker (1973) found it in the upper Miocene. In the present material $G$. bulloideus was found at Sites 253 and 254 in the middle Miocene, but at Sites 251 and 255 it ranged through the upper Miocene. Numerically it was never very frequent; in many samples only isolated specimens were encountered.

$G$. bulloideus is rather close to $G$. bollii; the latter differs from the former principally in being more compressed.

\section{Globigerinoides conglobatus (Brady, 1884)}

(Plate 4, Figures 16)

This species occurs in abundance only in upper Pliocene and Quaternary sediments at Site 253. Its first appearance is in the upper Miocene.

\section{Globigerinoides elongatus (d'Orbigny, 1826)}

(Plate 5, Figure 3)

Typical but scattered representatives of this species were found in lower Pliocene to Recent sediments.

\section{Globigerinoides fistulosus (Schubert, 1911)}

(Plate 5, Figure 16)

For convenience, following Parker's (1973) point of view, 1 interpret this foraminifer for this report as a species, although probably it would be biologically more correct to consider it as a form of Globigerinoides trilobus or to relate it to G. trilobus, f. sacculifera. It is an excellent marker species for middle and late Pliocene.

\section{Globigerinoides mitra Todd, 1957}

$$
\text { (Plate 5, Figures 1, 2) }
$$

This species is evidently a warm-water one as it was found only at the northernmost site (Site 253) where its vertical range was from lower Miocene to middle Miocene inclusive. The specimens found differ from the description and figures given by Todd in that supplementary apertures are somewhat larger than those in the primary types.
Globigerinoides obliquus Bolli, 1957, s.l. (Plate 5, Figures 4-6)

I share the point of view of Parker (1967) who lumped together $G$. obliquus obliquus and G. obliquus extremus. It was found in several sites from the lower and middle Miocene through the upper Pliocene.

\section{Globigerinoides pyramidalis (van den Broeck, 1876)}

(Plate 5, Figure 7)

The oldest records of this species are from the lower Pliocene (Site 258 ). I originally related this foraminifer to Globigerinoides ruber (Boltovskoy, 1969). Now I am inclined to consider it as much more closely related to $G$. elongatus. It is possible that it represents only a forma of the latter. Until a detailed study of this problem is carried out, I prefer to interpret it tentatively as a species.

Globigerinoides ruber (d'Orbigny, 1839)

This species is fairly frequent at all the sites studied. The lowest records are from the middle Miocene.

\section{Globigerinoides sicanus de Stefani, 1952 (Plate 5, Figures 8-12)}

According to Blow (1969), this species ranges from the lower Miocene to the base of the middle Miocene. According to Reiss and Gvirtzman (1966), it becomes extinct at the base of the upper Miocene.

At Sites 253-255 G. sicanus ranged up to the middle Miocene. However, at Site 258, typically developed specimens of this species were found through the middle Pliocene. The specimens found did not look reworked and as no other reworked species were found in these sediments, it is difficult to believe that $G$. sicamus found in the Pliocene section of Site 258 is resedimented. Thus, the upper limit of this species appears to be much higher than has been observed in other areas.

The following interesting morphological feature was observed in many specimens of $G$. sicanus recorded from the material of the present study. A very small, rounded, final chamber with its axis perpendicular to the main axis of the test was situated more or less in the middle of many specimens (Plate 5, Figures 11, 12). This final chamber partially covers the aperture. Probably it can be compared in function to the similar feature (abnormal last chamber) of Globigerina cryptomphala, G. tripartita, and several Globigerinita species described above.

\section{Globigerinoides tenellus Parker, 1958} (Plate 6, Figure 4)

Parker (1967) states that this is a typical Quaternary species which does not appear until the Pleistocene. Isolated specimens were found in the Quaternary at Sites 253 and 258.

\section{Globigerinoides trilobus (Reuss, 1850), s.l.} (Plate 5, Figures 13-15)

No separation was made between $G$. trilobus in Reuss interpretation and the foraminifer described in 1939 by Le Roy under the name of Globigerinoides sacculifer var. immatura. They were lumped together and considered as G. trilobus, s.l.

This is one of the most ubiquitous species in the Neogene deposits of the Indian Ocean. It was found at all the sites and in almost all the samples. Its lowest record is lower Miocene at Sites 250 and 253. However, specimens in many samples were small sized and not well developed. This is probably a result of too cool temperature during the Neogene in this part of the Indian Ocean for this warm-water dweller.

\section{Globigerinoides trilobus (Reuss), forma sacculifera (Brady, 1884)} (Plate 6, Figures 1, 2)

The reasons why this foraminifer is interpreted as a forma of $G$. trilobus, and not as an independent species (as it is considered by the majority of authors) have been discussed in a previous paper 
(Boltovskoy, 1971). This forma is less numerous than typical representatives of $G$. trilobus, and its first appearance is recorded in the present material somewhat later than the appearance of $G$. trilobus, forma typica, namely, in the middle rather than the lower Miocene.

\section{Globigerinopsis aguasayensis Bolli, 1962 \\ (Plate 6, Figures 5-14)}

Blow (1969) gives a very narrow range for this species, namely Zones N10 N12 only, which is the lower part of the middle Miocene.

Specimens referable to this species were found not only in the middle Miocene but also in the upper Miocene and in the uppermost part (Zone N8) of the lower Miocene.

\section{Globoquadrina altispira (Cushman and Jarvis, 1936}

(Plate 6, Figures 15, 16; Plate 7, Figure 1)

G. altispira has a high-spired test and usually five (less frequently four) chambers in the last coil. A relatively small number of specimens of this species was found in this study. Its stratigraphic range was throughout the Miocene and it became extinct in the middle Pliocene.

\section{Globoquadrina dehiscens dehiscens (Chapman, Parr, and Collins, 1934)} (Plate 7, Figures 2-4)

Undoubtedly this species has been described several times by different authors under different names. For junior synonymy see Parker (1967). The characteristic features of $G$. dehiscens by which it is distinguished from the closely related species, $G$. venezuelana, are the downward curving sides of the apertural face, a flat spiral side, and a somewhat less-rounded cross-section outline.

\section{Globoquadrina dehiscens praedehiscens Blow and Banner, 1962} (Plate 7, Figures 5-9)

This species is a marker of the upper Oligocene and lower Miocene. According to Blow (1969), it developed from Globigerina tripartita and was ancestral to Globoquadrina dehiscens dehiscens. Thus, the three forms mentioned should have much in common. G. dehiscens dehiscens and $G$. dehiscens praedehiscens are especially similar as they both belong to the same species. The latter differs from the former chiefly in having: (a) only three chambers in the final whorl, (b) a more triangular aperture, (c) a lower apertural face, and (d) a more rounded shape. There are several morphological differences which distinguish $G$. dehiscens praedehiscens from Globigerina tripartita, but the most important is that the former has apertural teeth.

It is interesting to note that among specimens of $G$. dehiscens praedehiscens there are many tests with an abnormal, poorly developed last chamber partially covering the aperture (Plate 7, Figures 7, 8). As mentioned above, a similar formation was observed in many foraminifera from the lower Miocene of the sites studied.

\section{Globoquadrina dutertrei (d'Orbigny, 1839)}

(Plate 7, Figure 10)

According to Parker (1967), $G$. dutertrei developed from $G$. humerosa in the uppermost Pliocene. Data obtained from the present study enlarge its range downward through middle Pliocene, although isolated specimens of $G$. dutertrei were found in middle Pliocene sediments at only one site (Site 258). At Sites 250 and 255 G. dutertrei is recorded from the upper Pliocene and Quaternary, respectively.

\section{Globoquadrina hexagona (Natland, 1938}

$$
\text { (Plate 6, Figure 3) }
$$

This is a rather typical Pliocene-Quaternary species. Isolated but characteristic specimens were recorded from the Pliocene sediments of Site 251.

\section{Globoquadrina humerosa (Takayanagi and Saito, 1962)} (Plate 7, Figure 15)

A very few specimens of this species were encountered in the upper Pliocene at Site 251.

\section{Globoquadrina venezuelana (Hedberg, 1937)}

(Plate 7, Figures 11-14)

According to Blow (1969), G. venezuelana and $G$. dehiscens both became extinct in the lower Pliocene. According to Parker (1967) and Postuma (1971), the latter species becomes extinct somewhat earlier than the former, namely $G$. dehiscens at the Miocene/Pliocene boundary or in the upper Miocene and $G$. venezuelana in the upper or middle Pliocene.

tl In the present study in the majority of the cases the same phenomenon could be observed; $G$. dehiscens had a shorter range than G. venezuelana. However, the youngest sediments in which both were found were middle Pliocene.

\section{Globorotalia acostaensis Blow, 1959}

(Plate 8, Figures 1-4)

This commonly used marker species is extremely scarce in the Indian Ocean and occurs very sporadically only at Site 251, so that no consistent range could be determined. A few specimens were more or less typical; many were tentatively considered as $G$. acostaensis.

\section{Globorotalia crassaformis (Galloway and Wissler, 1927), s.l.}

(Plate 8, Figures 5-11)

This is one of the most common upper Neogene species of the Indian Ocean. It was found at all the sites, sometimes very abundantly. Specimens referable to $G$. crassaformis are morphologically rather variable. It is very probable that a thorough and detailed study will prove that not one but several species make up the group which is called $G$. crassaformis in this report. Therefore, I prefer to use the name $G$. crassaformis, s.l.

There are some differences of opinion with respect to the range of $G$. crassaformis. Kennett (1966) preferred to correlate the first appearance of this species with the beginning of Pliocene. Parker (1967) stated that the lowest occurrence of $G$. crassaformis is found near the base of middle Pliocene. According to Blow (1969), its first occurrence is in the upper Miocene. In the material of the present study this species was first recorded at all the sites in the upper Miocene. At Sites 251 and 255 the same samples contained the lowermost appearance of both $G$. crassaformis and $G$. inflata. At Sites 250, 253, 254 , and 258 the former species appears in the upper Miocene, but somewhat earlier than $G$. inflata.

\section{Globorotalia crassula Cushman and Stewart, 1930} (Plate 8, Figures 12-16)

There is no unanimity in the interpretation of this species. In the present report I am following the viewpoint of Kaneps (1973) who wrote that $G$. crassula, in his interpretation, is very much like $G$. crassaformis but has a flattened test. He adds that "the test may be biconvex, concavo-convex or plano-convex and it may be unkeeled, partially keeled, or carinate" (Kaneps, 1973, p.737). This is also true with respect to my specimens, although the great majority of them can be described as biconvex with a spiral side less convex than the umbilical side. There are usually 4-4.5 chambers in the final whorl; however, 5 chambers can occur too. Poag (1972), and Jenkins and Orr (1972) presented figures of $G$. crassula which look like those found in the present study. According to the latter authors, the stratigraphic range of $G$. crassula in the eastern equatorial Pacific is upper MioceneRecent.

G. crassula was found in the upper Miocene-upper Pliocene section of Site 258

\section{Globorotalia crotonensis Conato and Follador, 1967}

(Plate 10, Figures 1-7)

This species was described from the middle Pliocene of Italy. Later it was found in the Pliocene of the Southwestern Atlantic (Boltovskoy, 1973) and in the middle Pliocene-Recent in the Tropical Atlantic (Parker, 1973).

In the present material, $G$. crotonensis has much similarity with $G$. miozea conoidea. However, G. miozea conoidea usually has a very highvaulted umbilical side and a less, but somewhat convex, dorsal side, and its ultimate and penultimate chambers exhibit a peripheral keel. 
G. crotonensis is not as convex, its periphery is rounded, and often it has a somewhat open umbilicus. In addition, these species occupy different stratigraphic positions. G. crotonensis is a good middle and upper Pliocene marker, and only isolated specimens of this species were found in the Quaternary section at Site 250. G. miozea conoidea is a typical Miocene species, although it ranges through Pliocene too. The species can sometimes be found together in Pliocene sediments. $G$. crotonensis may have developed from $G$. miozea conoidea.

Globorotalia hirsuta (d'Orbigny, 1839)

A few relatively rare specimens were recorded from PlioceneQuaternary sediments at Sites 255 and 258.

\section{Globorotalia cf. humilis (Brady, 1884)}

(Plate 7, Figure 16)

This foraminifer was tentatively ascribed to $G$. humilis because in some aspects it looks like that species; however, it differs considerably in having fewer chambers, very poorly defined sutures (often invisible), a smaller size, and a more elongated shape. It is a rather enigmatic foraminifer. Even after studying several thousand Recent plankton samples, I have found this species in Recent planktonic assemblages very seldom. However, I found it abundantly in the Quaternary sediments of the southwestern Atlantic (Boltovskoy, 1973) and in the upper Miocene-Quaternary deposits at Site 250. It is interesting to note that, concerning $G$. humilis, Ruddimann et al. (1970) also emphasized that this species was found in the North Atlantic cores but was not found in plankton tows.

\section{Globorotalia inflata (d'Orbigny, 1839)}

$$
\text { (Plate 9, Figures 1-8) }
$$

This is one of the most common species encountered in the material from Leg 26.

As stated previously, $G$. inflata is considered by many researchers as the best marker for the beginning of the Pliocene. It was used in this way in the present work. However, it should be emphasized that it is not the first appearance of $G$. inflata that signifies the lowermost Pliocene sediments, but the level at which this species becomes abundant or at least common. Naturally, this criterion can be used only in the temperate zone.

At Sites 250, 251, 254, and $258 \mathrm{G}$. inflata was found in the uppermost Miocene deposits (Zones N16-N18), but as very rare isolated specimens. At the same sites even the lowermost Pliocene samples possessed abundant populations of $G$. inflata.

It is also of interest to mention that although it is not difficult to separate G. inflata from G. crassaformis in Quaternary deposits, such is not the case in earlier deposits. In upper Pliocene deposits several kinds of transitional forms may be encountered and this makes the separation of these two species difficult. Among the population of $G$. inflata the number of specimens with four chambers in the final whorl, a flat dorsal side, an angular periphery, and less-vaulted ventral side increases. Simultaneously, Globorotalia crassaformis loses its lobulate peripheral outline and the truncate convexity of its umbilical side. As a result many specimens are found whose assignment to one or another species is extremely difficult. This situation grows worse downwards and in the lower Pliocene sediments the number of transitional forms becomes greater than the number of those whose identification is more or less straightforward.

The transformation of $G$. inflata and $G$. crassaformis described above is especially well illustrated at Site 254 .

In the Miocene deposits $G$. inflata exhibits a gradational series with G. miozea conoidea. This phenomenon was especially well observed at Site 251 . Site 253 yields an interesting picture of a gradational decrease upwards in the number of specimens of $G$. miozea conoidea and a corresponding increase in the number of $G$. inflata and $G$. crassaformis specimens.

Undoubtedly $G$. inflata and $G$. crassaformis are closely related species, and it is highly probable that McInnes (1965) was right when he stated that $G$. inflata evolved from $G$. miozea during the Miocene in the South Pacific and migrated to the Northern Hemisphere in early Pliocene time. Probably, it is still better to consider G. miozea as an ancestor not only of $G$. inflata, but also of $G$. crassaformis.

\section{Globorotalia kugleri Bolli, 1957}

(Plate 9, Figures 9, 10)

This is a good marker of short stratigraphic range. According to Blow (1969), it characterizes Zone N3 (the uppermost Oligocene) and Zone N4 (the lowermost Miocene).

Rather typical, but rare representatives of this species were found in the lower Miocene only at Site 253.

Globorotalia limbata (Fornasini, 1902)

(Plate 10, Figures 8-16; Plate 11, Figure 1)

In the interpretation of this species I accepted Parker's (1973) point of view that $G$. limbata is very closely related to $G$. multicamerata and that they can be distinguished by means of the following criterion: if a specimen has eight or more chambers in the final whorl, it should be considered as $G$. multicamerata; if less, G. limbata. Parker adds that "the present concept is a utilitarian one which may not be tenable from the point of view of a natural classification." However, this is the best way to handle the situation until the whole problem of the taxonomic position of both species is studied in detail.

G. limbata found in the Leg 26 material is characterized by its rather circular outline, by its chambers which increase somewhat (not very rapidly) in size as they are added, and by its deep open umbilicus. The sutures of this species on the dorsal side are usually limbate.

G. limbata is definitely dextral in the Pliocene. In the upper Miocene it abruptly changes its coiling direction to sinistral and goes back and forth from sinistral to dextral several times.

As an example of the changes coiling direction of $G$. limbata, the following observations can be described. At Site 253 the first appearance of this species was recorded in the lowermost sample of the upper Miocene; sinistral specimens were dominant at that time. The coiling direction subsequently changed abruptly four times; thus dextral coiling dominated twice, and sinistral coiling was predominanttwice. It should be emp0asized that in all cases the predominance was very strong, about $95 \%$ to $5 \%$ or more. Each period of dominance was observed to persist vertically over several meters (7$10 \mathrm{~m}$ ) of sections. With the beginning of the Pliocene, practically all the specimens b5came dextral and this coiling direction, without any change, lasted until the extinction of the species, which took place within the upper Pliocene.

G. limbata was found at three sites, 251,253, and 258. This indicates that it did not have any strong latitudinal preference. At all of the sites its stratigraphic range was from upper Miocene through upper Pliocene.

Globorotalia aff. limbata (Fornasini, 1902) (Plate 11, Figures 2-4)

This species was found in the middle Miocene sediments at Sites 254 and 255. It differs from $G$. limbata in having fewer chambers in the final whorl (only 5 or 6 ) and usually a more convex spiral side. Its outline is more rounded as it does not exhibit a lobing of the final part of the last coil. It can be supposed that $G$. aff. limbata is an ancestor of G. limbata.

\section{Globorotalia margaritae Bolli and Bermúdez, 1965} (Plate 9, Figures 13-16)

Thanks to the very typical last chamber, which looks from the umbilical side like a triangular "piece of pie," G. margaritae is an easily identifiable species. It has a relatively narrow keel anda rather convexly rounded spiral side.

At three sites $G$. margaritae was found in middle Miocene sediments. This record extends the stratigraphic range of this species considered by the majority of researchers (Blow, 1969; Postuma, 1971; Parker, (1973) as an indicator of deposits not older than upper Miocene.

\section{Globorotalia menardii (d'Orbigny, 1839) (sin), s.I.} (Plate 9, Figure 11)

For this report I lumped under the name $G$. menardii, s.l. specimens which can be considered as typical $G$. menardii and those which are 
interpreted by different authors as G. cultrata, G. tumida, and $G$. ungulata. This does not mean that I really consider them as one species. I do not wish to discuss here the rather complicated relationships existing among these species. Undoubtedly they are closely related and I lumped them together for convenience. Since the area under study is located mainly in the temperate zone, the majority of the cores contained only very rare isolated specimens of $G$. menardii, s.l. Citing them separately would give poor and unreliable data, especially if we take into account that in very many cases it was really very difficult to separate the species mentioned from numerous transitional forms.

\section{Globorotalia menardii (d’Orbignya 1839), forma flexuosa (Koch, 1923)}

(Plate 9, Figure 12)

This foraminifer is treated separately and was not included in $G$. menardii, s.l. for the following reasons: (a) it is a very easily identifiable taxon, (b) it has been used by several authors as a marker species.

It was first recorded and described by Koch (1923) as a subspecies of $G$. tumida. The tendency to create a forma flexuosa in $G$. tumida was mentioned also by Parker (1967). However, forma flexuosa is widely applied also to G. menardii. Ericson et al. (1964), for instance, interpreted this foraminifer as a subspecies of $G$. menardii and stated that it became extinct during the main Wisconsin epoch. Boltovskoy (1968), Bé and McIntyre (1970), and Adegoke et al. (1971) found it in the Recent plankton of the Atlantic and Indian oceans. The facts that (a) forma flexuosa can be found among $G$. menardii and $G$. tumida, (b) its records are known interruptedly from within the Miocene to the Recent, and (c) that the mreatest percentages oRforma flexuosa were observed in the populations of $G$. menardii characterized by many aberrant forms testify to the supposition that forma flexuosa is a result of some environmental influence. Lidz (1966) states that aberrant specimens of $G$. menardii can be formed at abnormally high temperatures. Bé and McIntyre (1970) do not accept this explanation as sufficient, and the data from the present study do not confirm the supposition of Lidz either.

In the material studied $G$. menardii ( $\sin )$, s.l. ranges from upper Miocene to Recent.

\section{?Globorotalia miocenica Palmer, 1945}

(Plate 12, Figures 1-3)

This is a rather common species in zhe Neogene sediments of the Indian Ocean. It ranges from middle Miocene through middle Pliocene. It was tentatively ascribed to G. miocenica: however, being not sure of this identification, a question mark has been left. Specimens are rather large and planoconvex. They have a flat dorsal side, a characteristic which indicates a close relationship with typical specimens of $G$. miocenica. However, other characteristics, such as, for example, a smaller number of chambers in final whorl, a highly vaulted last chamber on the ventral side, and some others, distinguish this species from $G$. miocenica.

\section{Globorotalia miozea conoidea Walters, 1965 (Plate 11, Figures 5-18)}

G. miozea conoidea was one of the most common species in the Miocene and lower Pliocene sediments, especially at Sites 254 and 258. It was also recorded in sediments as high as middle Pliocene at Sites 251 and 254, and in the upper Pliocene at Site 258. For remarks on its morphological appearanc5, see the discussion of $G$. crotonensis.

$G$. miozea conoidea, according to researchers from New Zealand (Walters, 1965; McInnes, 1965; Hornibrook, 1967; Jenkins, 1971), is a typical Miocene marker. Olsson (1971) gives it a range from Miocene to Pliocene. The records of the present study extend upward considerably the stratigraphic range of $G$. miozea conoidea.

\section{Globorotalia opima Bolli, 1957, s.l.}

(Plate 12, Figures 4-8)

It was difficult to separate $G$. opima opima from $G$. opima nana. Both foraminifers have all kinds of transitional forms. They were therefore combined under G. opima, s.l.
Specimens ascribed to this species were recorded at all sites (nowhere very frequently) mostly in lower Miocene sediments. At Sites 251 and 255 it was also recorded in middle Miocene sediments and at Sites 253 and 254 it was found in Oligocene deposits.

These data contradict the widely accepted interpretation of $G$. opima as a typical Oligocene species. The specimens found in Miocene deposits did not have the appearance of being reworked. They were found rather continuously in the section at all the sites with the same assemblage. This proves that they were in situ. Thus, it seems that the upper limit of G. opima, s.l. should be considerably raised up to the middle Miocene at least, for the Indian Ocean.

Finds of G. opima, s.l. in Miocene sediments are not new. Soediono (1970) recorded G. opima nana from the Miocene deposits in Spain and Baumann (1972) recorded specimens in Borneo.

\section{Globorotalia peripheroacuta Blow and Banner, 1959}

(Plate 12, Figures 9-11)

This species was found in the middle Miocene sediments at Sites $251,253-255$, in very limited numbers.

\section{Globorotalia peripheroronda Blow and Banner} (Plate 12, Figures 13, 14)

$G$. peripheroronda was found at the same sites as the foregoing species and also at Site 250. Its range is somewhat wider, and it is somewhat more numerous than $G$. peripheroacuta. Both species are excellent lower and middle Miocene markers because they are easily identifiable and have a relatively short stratigraphic range.

\section{Globorotalia petaliformis n. sp.}

(Plate 14, Figures 1-16)

Description: Test free, medium in size compared with other species of Globorotalia, trochospiral, sinistrally coiled, biconvex, the umbilical side considerably more convex than the spiral side (rare specimens are equally biconvex). There are also planoconvex tests in which the spiral side is flat; equatorial periphery almost circular; peripheral outline strongly lobate. Axial periphery angled, sometimes rather round. Chambers angular, rhomboid, about 13-16 in the whole test arranged in 2.5-3 coils. Early whorl sometimes raised above the subsequent ones. Five chambers compose the final whorl; often they are offset from each other creating an imbricate appearance on the spiral side. Sutures variable from simple to strongly limbate, sometimes raised; curved on the spiral side, curved or radial, and depressed on the umbilical side. Umbilicus can be closed or narrow and deep. Aperture arched, interiomarginal, extraumbilical-umbilical, bordered by a rim. Wall calcareous, moderately thick, fairly smooth, rather coarsely perforate, sometimes pustulate on the umbilical side near the aperture. The largest diameter $0.25-0.45 \mathrm{~mm}$. The height of the test $0.16-0.30$ $\mathrm{mm}$.

Occurrence: Globorotalia petaliformis n. sp. occurred abundantly in the middle Miocene deposits of Site 254 and as rare or isolated specimens in middle Miocene of Sites 253 and 255, and in upper Miocene of Sites 253-255.

Diagnosis and comparison: The most striking features of Globorotalia petaliformis n. sp. are the very lobate peripheral outline and the often step-like sutures on the spiral side which give an imbricate appearance. Because of these characteristics, the test of this species resembles a flower; hence the name petaliformis (petalled).

It is a very variable species, especially concerning its biconvexity, the character of the sutures, the kind of angularity of the peripheral margin, and its imbricate appearance. However, it is a very peculiar and easily identifiable foraminifer which is not closely related to any Globorotalia as yet described. Some tests of Globorotalia petaliformis n. sp., because of the imbricate appearance, resemble the Recent coldwater species Globorotalia cavernula Bé.

Type level: Middle and upper Miocene of the middle part of the Indian Ocean.

\section{Type specimens:}

Holotype: (Plate 14, Figures 1a, 1b) from core catcher $10(10, \mathrm{CC})$ at Site 254. Depth from the sea bed, 91 meters. Dimensions of the 


\section{E. BOLTOVSKOY}

holotype: greatest diameter $0.40 \mathrm{~mm}$; height $0.21 \mathrm{~mm}$. Deposited in the Foraminiferal Collection of the Museo Argentino de Ciencias Naturales "Bernardino Rivadavia" under number FMACN 7646.

Paratypes: About 400 specimens from the middle and upper Miocene of Sites 253, 254, 255, and 258 of L5g 26 of the DSDP. Deposited in the same collection under number FMACN 7647.

Type locality: Holotype: Leg 26, Hole 254, DSDP. Lat $30^{\circ} 58.15^{\prime} \mathrm{S}$, long $87^{\circ} 53.72^{\prime} \mathrm{E}$. Water depth 1253 meters. Depth from the sea floor 91 meters.

Observations: I prefer to establish it as a new taxon but not to put it in open nomenclature because it is a very peculiar, typical, and easily identifiable species which, in addition, has a relatively short stratigraphic range. In spite of all my efforts to find at least a related species, I was not able to find any.

\section{Globorotalia pseudobulloides (Plummer, 1926)} (Plate 13, Figures 2, 3)

Plummer (1926) described this species from the Eocene sediments of Texas. Rather typical specimens were found in Oligocene sediments at Site 253.

\section{?Globorotalia pseudomiocenica Bolli and Bermúdez, 1965}

$$
\text { (Plate 12, Figures 12, 15, 16) }
$$

G. pseudomiocenica, according to Bolli and Bermúdez (1965), differs from $G$. miocenica mainly in its slightly convex spiral side and somewhat more lobulate periphery. The form recognized here is assigned only tentatively to $G$. pseudomiocenica.

\section{Globorotalia cf. pseudopima Blow, 1969}

(Plate 13, Figure 4)

According to Blow (1969, p.387), in Globorotalia (Turborotalia) acostaensis pseudopima the aperture "extends slightly beyond the midplane of the periphery and thus encroaches slightly onto the dorsal side." In specimens tentatively named $G$. cf. pseudopima the aperture is situated completely on the ventral side. Other morphological characteristics of both foraminifers are identical. Their stratigraphical ranges, however, are not identical. G. pseudopima was recorded from the base of Zone N20 (middle Pliocene) to the Recent. I found specimens ascribed to $G$. cf. pseudopima only atSite 251 , from the upper Miocene to upper Pliocene.

\section{Globorotalia scitula (Brady, 1882)} (Plate 13, Figure 1)

This species was recorded only at Site 258 where its occurrence was almost uninterrupted from the upper Miocene to Recent. The specimens are small but typical.

\section{Globorotalia tosaensis Takayanagi and Saito, 1962}

(Plate 13, Figures 5-7)

The importance of this species as an indicator for the location of the Pliocene/Quaternary boundary, as well as its stratigraphic range, was discussed previously.

\section{Globorotalia truncatulinoides (d'Orbigny, 1839)}

(Plate 13, Figures 9-11)

This species was found at all the sites; the lowest records were from upper Pliocene sediments. Details of its range were discussed previously.

\section{Orbulina suturalis Bronnimann, 1951 (Plate 13, Figure 8)}

According to Reiss and Gvirtzman (1966), O. suturalis ranges in Israel from the lower Miocene into the upper Miocene. The same range was found at the Leg 26 sites. This range is much more limited than that presented by Blow (1969), namely, from the lowermost middle Miocene up to Recent.

Specimens were small sized and, in the majority of the samples, quantitatively rare.

\section{Orbulina universa d'Orbigny, 1839}

This is one of the most common species in the material studied. It was recorded at all the sites but was especially abundant at Sites 253, 254 , and 258. Its stratigraphic range in Leg 26 material is from middle Miocene to Recent.

\section{Praeorbulina glomerosa circularis (Blow, 1956)}

This species, according to Blow (1969), has a very short stratigraphic range, occurring only in Zones N8 (uppermost lower Miocene) and N9 (lowermost middle Miocene). It is a very characteristic species whose identification does not present any problem. It was found at two sites: 251 and 253 . However, although at the latter site its range corresponds perfectly with the data of Blow, at Site 251 isolated specimens occurred much lower (down to Zone N5) and much higher (up to Zones N13-N15) in the section.

\section{Pulleniatina obliquiloculata (parker and Jones, 1862), and related spp. (Plate 13, Figure 13)}

Banner and Blow (1965) studied species referable to the genus Pulleniatina, redescribed existing taxa, established some new ones, and detailed the stratigraphic ranges of all of them. They compiled the following table.

Pulleniatina primalis Banner and Blow: Zones N17-N20

Pulleniatina obliquiloculata praecursor Banner and Blow: Zones N19-N21

Pulleniatina obliquiloculata (Parker and Jones), s.s.: Zones N22Recent .

Pulleniatina obliquiloculata finalis Banner and Blow: Zones N22Recent.

Of all these taxa the following three were found in the Leg 26 material:

$P$. primalis (Zones N19-N20; Site 253), $P$. obliquiloculata praecursor (Zones N22-N23, Site 253; Zones N20-N23, Site 258), and $P$. obliquiloculata, s.s. (Zones N21-N23, Site 250; Zones N20-N23, Site 258).

\section{Sphaeroidinella dehiscens (Parker and Jones, 1865)} (Plate 13, Figure 15)

This species is interpreted by many authors as an excellent marker of the Miocene/Pliocene boundary. Above this boundary $S$. dehiscens can be found, and below it, its ancestor $S$. subdehiscens is found.

$S$. dehiscens was recorded at all sites, never very frequently, and only in the Pliocene/Quaternary sediments.

\section{Sphaeroidinella seminulina (Schwager, 1866)} (Plate 13, Figures 14, 16, 17)

This is very variable species. Its variability was discussed by Parker (1967). According to Blow (1969), it ranges from lower Miocene to lower Pliocene. Parker (1967) raises the position of its extinction to the middle Pliocene.

$S$. seminulina was found at all sites with the exception of the westernmost one (Site 250). Its stratigraphic range is lower Miocenemiddle Pliocene. In the upper part of its range this species is characterized by a very coarsely cancellated test surface. In several cases it was very difficult to distinguish this species from $S$. subdehiscens. Kaneps (1973, p. 735$)$ states that the only difference is "a final pointed, oblique chamber in $S$. seminulina, resulting. in four chambers in the final whorl."

\section{Sphaeroidinella subdehiscens Blow, 1959 (Plate 13, Figure 12)}

This taxon was separated by Blow from $S$. dehiscens on the basis of the following differences: absence of supplementary apertures, less embracing chambers, more visible sutures, generally smaller test, and more lobate equatorial periphery. It is typically a Miocene species; however, it can range through the Pliocene (Parker, 1973). Rare specimens of it were recorded at Sites 251, 253, 254 and 255 from the upper Miocene to lower/middle Pliocene. 


\section{INDIAN OCEAN FROM MIOCENE TO RECENT}

The Miocene climate is considered to have been warm; however, according to several authors, during the upper Miocene a period of low temperatures took place. Kennett (1968) stated that at that time the Antarctic Convergence occupied a much more northerly position than now (approximately $39^{\circ} \mathrm{S}$ ). At the present its position is about $60^{\circ} \mathrm{S}$.

Bandy (1969) also found that during the upper Miocene a cold period occurred in several areas. This is proved, according to Bandy et al. (1971), by the presence of Globigerina bulloides in tropical areas, of Globigerina pachyderma (sin.) in the temperate zone, and of glacial sediments in the Antarctic.

Hays (1969) found that in the Miocene, approximately $10 \mathrm{~m} . \mathrm{y}$. ago, a glacial epoch took place in Alaska.

The simplest way to estimate the surface-water temperature of the past geological epochs using planktonic foraminifera is to compare the fossil assemblages with Recent ones of different climatic zones. This method has three difficulties: (a) comparison should be made on the basis of living species because we do not know the temperature requirements of extinct species. It is well known that the older the assemblage, the fewer the number of species in common with the Recent fauna; (b) even when comparing the lists of living species we are not sure that a species, which lives in certain temperature limits now, had the same limits in the past; (c) the dissolution effect can strongly change any fossil assemblage.

There are some additional criteria which can be used to reach paleotemperature conclusions based on foraminifera. For instance, a warm-water fauna is usually much richer qualitatively than a cold-water one; the representatives of genus Globigerinoides are mostly warm-water species, etc. Unfortunately the applicability of these criteria is also dependent on dissolution effects.

The vertical distribution of fauna encountered at the sites studied does not show any dramatic change during the Miocene. The sedimentation rate also was close to that for the Recent.

As for benthonic foraminifera, time did not permit a detailed study. However, many specimens were picked out and examined. They did not show any changes which could be attributed to the results of major changes of depth. Nevertheless, it should be taken into account that in deep-water depth changes are very difficult to detect by means of benthonic foraminifera. Deep-water benthonic foraminifera are still insufficiently studied to be used for this kind of study and many species have a large depth range. However, other observations indicate that ocean depths or the depth of the lysocline changed during the Miocene in the area of our study, at least at Sites 251 and 253. The foraminiferal assemblages of the early Miocene are strongly affected by dissolution at both sites mentioned. These assemblages are less affected in middle and late Miocene, and the foraminiferal fauna is well preserved in the Pliocene sediments. The most logical explanations of this phenomenon are that either depths were greater in the Miocene, or that the lysocline was shallower, whereas during the Pliocene, either the water was shallower or the lysocline was deeper, and thus, dissolution could not have affected foraminiferal tests. These changes were gradual and depths were always at least bathyal during the Pliocene.

Thus, we can conclude that the circulation system of oceanic currents, the general character of the depths, and climatic zonation during the Miocene time were approximately the same as now. The mean temperature, however, was probably somewhat higher than at the present. Only in the late Miocene did a relatively short period of low temperature take place in the Indian Ocean. This temperature decrease was mentioned for other areas previously by Kennett (1968), Bandy (1969), and Hays (1969). It is shown in the present material by a considerable decrease in abundance of such typical warm-water species as Globigerinoides trilobus, s.l., G. ruber and, to a certain degree, Globorotalia menardii, s.l. The latter species is relatively rare in the area under study and therefore is not so useful as the two former species. While the warm-water species decreased, the Globigerina bulloides group (cold and cold-temperate indicator) increased in number. But even at the southernmost site (Site 251, lat $36^{\circ} 30.26^{\prime} \mathrm{S}$ ), no specimens of a typical cold-water indicator- a sinistrally coiled Globigerina pachyderma-were found, and warm-water Globigerinoides conglobatus, G. trilobus, G. ruber still lived, although as rare individuals. This means that the area was still in the Temperate Zone.

In the description of Site 251 above, a lower Miocene assemblage of benthonic foraminifera of quite shallow water is recorded. It is suggested, however, that this assemblage is the result of sediment transport, but not of dramatic changes of oceanic depth in this area. Unfortunately no data were available to determine more exactly the origin of this benthonic assemblage.

As for the Pliocene, it can be suggested that the average temperature of the surface water during this time was a little lower than in the Miocene. In addition, according to various authors, a cold period (or periods) also occurred, and several authors consider that it was still colder than that which took place in the Miocene.

Bolli et al. (1968), for example, after studying Pliocene sediments in the Caribbean Sea, recognized at least two glacial periods there.

Bandy (1969) stated that in the middle Pliocene the surface-water temperatures in the temperate zone were similar to those found during Pleistocene glacial epochs, namely between $2^{\circ} \mathrm{C}$ and $8^{\circ} \mathrm{C}$.

Bermúdez and Bolli (1969) are of opinion that in the lower Pliocene the water temperatures of the oceans were lower than at present.

Kennett et al. (1971) found two glacial epochs in the Pliocene of New Zealand, one in the middle Pliocene and the other in the upper Pliocene.

Boltovskoy (1973) also found that in the southwestern Atlantic the Pliocene was a period with temperatures similar to those of the glacial epochs of the Pleistocene, i.e., considerably lower than the present day. 
The study of the Leg 26 material revealed fluctuations of temperature during the Pliocene at some sites; however, with the exception of Site 253, these fluctuations were very insignificant and it would not be correct to interpret them as an effect of real glacial epochs.

At Site 253, at a depth of 8 meters below the bottom surface, the foraminiferal assemblage indicated a temperature decrease. The evidences of lower temperatures are an increase in the number of specimens of Globorotalia truncatulinoides/tosaensis, a decrease in the number of specimens of G. menardii, s.l., Globigerinoides ruber, etc. This temperature decrease can be seen still better in a detailed climatic curve prepared using samples taken at $10-\mathrm{cm}$ intervals (see Boltovskoy, late Pliocene and Quaternary paleoclimatic changes, this volume). No temperature decrease was observed in the middle Pliocene.

The Pleistocene is a time of glacial/interglacial oscillations. Usually one considers that glaciation began at the beginning of the Pleistocene; however, there are many authors who do not correlate these events.

Since a separate paper is devoted to Quaternary temperature changes, it would be out of place to go into details here. It can be stated that the Quaternary temperature oscillations were not sufficiently well pronounced to be correlated with known glacial and interglacial epochs.

An analysis of the Pliocene-Recent planktonic foraminiferal assemblages, as well as of the benthonic foraminiferal fauna, proved that during the Pliocene, just as during the Miocene, oceanographical conditions 'in the area under study hardly changed.

The following minor biological phenomenon is of interest with reference to the Indian Ocean during the Miocene. Several Globigerina ( $G$. cryptomphala, $G$. tripartita, G. unicava), Globigerinita (G. cf. africana, $G$. dissimilis dissimilis, G. dissimilis ciperoensis), and even Globoquadrina ( $G$. dehiscens praedehiscens) developed an interesting structure, a poorly developed bulla-like last chamber which, like a valve, partially covered the aperture. In some of the species listed it is well known and is a characteristic feature. However, in others it is observed for the first time. Also, as far as the author knows, the small final chamber which covers the aperture of Globigerinoides sicanus described in this report has not previously been observed. The main function of this structure apparently is to protect the aperture from predators and parasites.

The great development of foraminifera with this protection in the Miocene suggests that foraminiferal predators were common at that time in the Indian Ocean.

\section{ACKNOWLEDGMENTS}

I wish to express my sincere thanks to the following institutions and persons:

1) The authorities of the Deep Sea Drilling Project for their kind invitation to participate onboard the Glomar Challenger for Leg 26 and for financial support to spend several days visiting the Smithsonian Institution.
2) Drs. N. de B. Hornibrook, G. H. Scott, R. Hoskin, P. Vella (all of them in Wellington), Frances L. Parker (La Jolla), and Ruth Todd (Washington) for their valuable opinions with respect to the taxonomic positions of several species.

3) Dr. T. A. Davies (La Jolla) for his help in the editing of the manuscript.

4) Dr. P. Bermúdez (Caracas) for an excellent collection of Neogene planktonic foraminifera.

5) Mr. J. Remiro, Mrs. I. C. Riobo de Magaldi, Miss A. M. Leverone, and Miss S. Watanabe (all of them from Buenos Aires) for their technical help without which I would not have been able to finish the study of such a great number of samples in a relatively short time span.

6) Mr. N. De Vicenzo and Scanning Electron Microscope Service of the CONICET (Argentina) for taking all the photomicrographs.

\section{REFERENCES OF RECORDED PLANKTONIC FORAMINIFERA}

Candeina nitida d'Orbigny, 1839, In de la Sagra, Foraminiferes. Hist. Phys. Pol. Natl. Cuba, p. 108, pl. 2, fig. 27, 28.

Globigerina ampliapertura Bolli, 1957. U.S. Natl. Museum, Bull. 215 , p. 108 , p1. 22, fig. 4-7.

Globigerina angiporoides Hornibrook, 1965. New Zealand J. Geol. Geophys., v. 8, p. 835, fig. 1,2.

Globigerina angulisuturalis Bolli $=$ Globigerina ciperoensis angulisuturalis Bolli, 1957. U.S. natl. Museum, Bull. 215, p. 109 , pl. 22, fig. 11.

Globigerina bulloides d'Orbigny, 1826. Ann. Sci. Natl., Paris, v. 7, Mod. 17 and 76; Banner and Blow, 1960. Cushman Found. Foram. Res. Contrib. v. 11, p. 3, pl. 1, fig. 1, 4 (lectotype).

Globigerina calida Parker, 1962. Micropaleontology, v. 8, p. 221, pl. 1, fig. 9-13, 15.

Globigerina cryptomphala Glaessner = Globigerina bulloides d'Orbigny var. cryptomphala Glaessner, 1937. Moscow Univ. Paleontol. Lab. Studies Micropaleontol. v. 1, fasc. 1, p. 29 , pl. 1, fig. 1 .

Globigerina decoraperta Takayanagi and Saito $=$ Globigerina druryi Akers decoraperta Takayanagi and Saito, 1962. Tohoku Univ. Sci. Rept. Second Ser. (Geol.), Sp. v. 5, p. 85 , pl. 28, fig. 10.

Globigerina euapertura Jenkins, 1960. Micropaleontology, v. 6, p. 351 , pl. 1, fig. 8 .

Globigerina falconensis Blow, 1959. Am. Paleontol. Bull., v. 39, p. 177 , pl. 9 , fig. 40,41 .

Globigerina juvenilis Bolli, 1957. U.S. Nat1. Museum, Bull. 215, p. 110 , pl. 24 , fig. 5 , 6 .

Globigerina linaperta Finlay, 1939. Roy. Soc. New Zealand Trans., Proc., v. 69, p. 125, pl. 13, fig. 54-57.

Globigerina nepenthes Todd, 1957, U.S. Geol. Surv., Prof. Paper 280-H, p. 301, pl. 78, fig. 7.

Globigerina pachyderma (Ehrenberg) = Aristerospira pachyderma Ehrenberg, 1861. Kgl. Preuss. Akad. Wiss. Berlin, Monatsber., p. 303; 1873. Kgl. Akad. Wiss. Berlin, Abhandl., Jahrg, 1872. p. 386, pl. 1, fig. 4.

Globigerina quinqueloba Natland, 1938. Scripps Inst. Oceanogr. Bull., Tech. Ser., v. 4, p. 149, pl. 6, fig. 18-21.

Globigerina rubescens Hofker, 1956. Copenhagen Univ. Zool. Museum, v. 15 , p. 234 , pl. 35 , fig. 18-21.

Globigerina tripartita Koch $=$ Globigerina bulloides d'Orbigny var. tripartita Koch, 1926. Ecolog. Geol. Helv, v. 19, p. 746, fig. $21 \mathrm{a}, \mathrm{b}$.

Globigerina yeguaensis Weinzierl and Applin, 1929. J. Paleontol. v. 3, p. 408, pl. 43, fig. 1. 
Globigerinella aequilateralis (Brady) $=$ Globigerina aequilateralis Brady, 1879. Quart. J. Micros. Sci., v. 19, p. 285; 1884. Rept. Voy. Challenger, Zool., v. 9, p. 605, pl. 80, fig. 18-21.

Globigerinita africana Blow and Banner, 1962. In: Eames, Banner, Blow, and Clarke Fundamental mid-Tertiary stratigraphic correlations, Cambridge (Cambridge Univ. Press), p. 105, pl. 15, fig. A-C.; p. 103, fig. 11, I-IV.

Globigerinita dissimilis ciperoensis Blow and Banner, 1962. In Eames, Banner, Blow, and Clarke, p. 107, pl. 14, fig. A-C.

Globigerinita dissimilis dissimilis (Cushman and Bermudez) = Globigerina dissimilis Cushman and Bermudez, 1937. Cushman Lab. Foram. Res. Contrib., v. 13, pt. 1, p. 25, pl. 3, fig. 4-6.

Globigerinita glutinata $($ Egger) $=$ Globigerina glutinata Egger, 1893. Abhandl. Kgl. Bayer. Akad. Wiss., cl. II, v. 18, p. 371, pl. 13, fig. 19-21.

Globigerinita unicava (Bolli, Loeblich, and Tappan) = Catapsydrax unicavus Bolli, Loeblich and Tappan, 1957. U. S. Natl. Museum, Bull. 215, p. 37, pl. 7, fig. 9.

Globigerinoides amplus Perconig $=$ Globigerinoides obliquus amplus Perconig, 1968. Gior. Geol., Bologna, ser. 2, v. 35, p. 225 , pl. 7 , fig. $20-22$.

Globigerinoides bulloideus Crescenti, 1966. Geol. Rom., Rome, v. 5 , p. 43 , fig. 8 , p. 43 . fig. 9 .

Globigerinoides conglobatus $($ Brady $)=$ Globigerina conglobata Brady, 1879. Quart. J. Micros. Sci., vol. 19, p. 186; 1884. Rept. Voy. Challenger. Zool., v. 9, pl. 80, fig. 1-5; pl. 82, fig. 5.

Globigerinoides elongatus (d'Orbigny) = Globigerina elongata d'Orbigny, 1826. Tabl. Meth., Ann. Sci. Nat., Paris, ser. 1, v. 7, p. 277; Fornasini, 1889. Paleontology Italy, v. 4, p. 207 , fig. 1.

Globigerinoides fistulosus (Schubert) $=$ Globigerina fistulosa Schubert, 1910. Geol. Reichsanst. Verhandl. Wien, p. 323, fig. 2; 1911. Geol. Reichsanst. Abhandl., v.20, H. 4, p. 101, fig. 13 .

Globigerinoides mitra Todd, 1957. U.S. Geol. Surv., Prof. Paper 280-H, p. 302, pl. 78, fig. 3.

Globigerinoides obliquus Bolli, 1957. U.S. Natl. Museum, Bull. 215 , p. 113 , pl. 25 , fig. 9 , 10; p. 112 , fig. 21.

Globigerinoides pyramidalis $($ van den Broeck $)=$ Globigerina bulloides, var. rubra. subvar. pyramidalis van den Broeck, 1876. Belgian Soc. Micros., Ann., v. 2, pl. 78.

Globigerinoides ruber (d'Orbigny) $=$ Globigerina rubra d'Orbigny, 1839, In de la Sagra, Foraminiferes: Hist. Phys. Pol. Nat. Cuba, p. 82, pl. 4, fig. 12-14.

Globigerinoides sicanus de Stefani, $1952=$ Globigerinoides conglobatus (Brady), Cushman and Stainforth, 1945. Cushman Lab. Foram. Res., Spec. Publ. 14, p. 68, pl. 13, fig. $6=$ Globigerinoides sicana de Stefani, 1952. Plinia, Palermo, v. 3 , nota 4 , p. 9.

Globigerinoides tenellus Parker, 1958. Swedish Deep Sea Exped. 1947-48: Rept., fasc. 2, p. 280, pl. 6, fig. 7-11.

Globigerinoides trilobus (Reuss) = Globigerina triloba Reuss, 1850. Klg. Akad. Wiss. Wien, Denkschr., v. 1, p. 474, pl. 47 , fig. 11 .

Globigerinoides trilobus (Reuss), forma sacculifera (Brady) = Globigerina sacculifera Brady, 1877. Geol. Mag., vol. 4, pl. 535; 1884. Rept. Voy. Challenger, Zool., v. 9, p. 604, pl. 80, fig. 11-17; pl. 82 , fig. 4

Globigerinoides aguasayensis Bolli, 1962. Ecolog. Geol. Helv., v. 55 , p. 282 , pl. 1 , fig. 1-7.

Globoquadrina altispira (Cushman and Jarvis) = Globigerina altispira Cushman and Jarvis, 1936. Cushman Lab. Foram. Res. Contrib., v. 12, pt. 1, p. 5, pl. 1, fig. 13, 14 .
Globoquadrina dehiscens (Chapman, Parr, and Collins) = Globorotalia dehiscens Chapman, Parr, and Collins, 1934. Linn. Soc. London (Zool.), v. 38, p. 569, pl. 11, fig. 36.

Globoquadrina dehiscens praedehiscens Blow and Banner, 1962. In: Eames, Banner, Blow and Clarke, Fundamental MidTertiary Stratigraphic Correlations, Cambridge (Cambridge Univ. Press), p. 116, pl. 15, fig. Q-S.

Globoquadrina dutertrei (d'Orbigny) = Globigerina dutertrei d'Orbigny, 1839. In de la Sagra, Foraminiferes: Hist. Phys. Pol. Nat. Cuba, p. 84, pl. 4, fig. 19-21; Banner and Blow, 1960. Cushman Found. Foram. Res. Contrib., v. 11, p. 12, fig. 1 .

Globoquadrina hexagona (Natland) = Globigerina hexagona Natland, 1938. Scripps Inst. Oceanogr. Bull., Tech. Ser., v. 4 , p. 149 , pl. 7 , fig. 1 .

Globoquadrina humerosa (Takayanagi and Saito) = Globorotalia humerosa Takayanagi and Saito, 1962. Tohoku Univ. Sci. Rept. Second Ser. (Geol.), Sp. v. 6, p. 78 , pl. 28, fig. 1, 2.

Globoquadrina venezuelana (Hedberg) = Globigerina venezuelana Hedberg, 1937. J. Paleontol., v. 11, p. 681, pl. 92 , fig. 7.

Globorotalia acostaensis Blow, 1959. Am. Paleontol., Bull. v. 39 , p. 208 , pl. 17 , fig. 106.

Globorotalia crassaformis (Galloway and Wissler) = Globigerina crassaformis Galloway and Wissler, 1927. J. Paleontol., v. 1 , p. 41 , pl. 7 , fig. 12 .

Globorotalia crassula Cushman and Stewart, 1930. San Diego Soc. Nat. Hist. Trans., v. 6, p. 77, pl. 7, fig. 1.

Globorotalia crotonensis Conato and Follador, 1967. Italian Soc. Geol. Boll., v. 86, fasc. 3, p. 556, fig. 1a-Ic (p. 557), fig. $1 \mathrm{a}-1 \mathrm{c}, 2 \mathrm{a}-2 \mathrm{c}$ (p. 560).

Globorotalia hirsuta (d'Orbigny) = Rotalina hirsuta d'Orbigny, 1839. In Barker-Webb et Berthelot, Hist. Natl. Iles Canaries, "Foraminiferes," v. 2, pt. 2, Zool, p. 131, pl. 1, fig. 37-39.

Globorotalia humilis (Brady) = Truncatulina humilis Brady, 1884. Rept. Voy. Challenger, Zool., v. 9, p. 665, pl. 94, fig. 7.

Globorotalia inflata (d'Orbigny) = Globigerina inflata d'Orbigny, 1839. In Barker-Webb et Berthelot, Hist. Natl. Iles Canaries, "Foraminiferes," v. 2, pt. 2, Zool., p. 134, pl. 2, fig. 7-9.

Globorotalia kugleri Bolli, 1957. U.S. Natl. Museum Bull. 215, p. 118 , pl. 28 , fig. 5,6 .

Globorotalia limbata $($ Fornasini) = Rotalia limbata d'Orbigny, 1826. Ann. Sci. Natl., Paris, Ser. 1, v. 7, p. 274 (nomennudum) = Rotalia limbata d'Orbigny, in Fornasini, 1902. Roy. Accad. Sci. Inst. Bologna, Mem. Sci. Natl. Ser. 5, v. 10 , p. 56 , fig. 55 .

Bloborotalia margaritae Bolli and Bermudez, 1965. Venezuelan Asoc. Geol. Min. Petr. Bol. Inform., v. 8, p. 139, pl. 1, fig. 16-18.

Globorotalia menardii (d'Orbigny) = Rotalia menardii d’Orbigny, 1826. Ann. Sci. Natl., Paris, v. 7, p. 273, Mod. 10.

Globorotalia menardii (d'Orbigny, forma flexuosa (Koch) = Pulvinulina tumida flexuosa Koch, 1923. Ecolog. Geol. Helv., p. 357, fig. 9, 10.

Globorotalia miocenica Palmer = Globorotalia menardii miocenica Palmer, 1945. Am. Paleontol. Bull. v. 29, p. 70, pl. 1, fig. 10 .

Globorotalia miozea conoidea Walters, 1965. New Zealand J. Geol. Geophys., v. 8, p. 124, fig. 8, I-M.

Globorotalia opima Bolli, 1957. U.S. Natl. Museum, Bull. 215, p. 117 , pl. 28 , fig. 1,2 . 
Globorotalia peripheroacuta Blow and Banner, 1959 Paleontology, v. 12, p. 22, pl. 1, fig. 2.

Globorotalia peripheroronda Blow and Banner,

Globorotalia petaliformis Boltvskoy, this report.

Globorotalia pseudobulloides (Plummer) $=$ Globigerina pseudobulloides Plummer, 1926. Texas Univ. Bull. 2644, p. 133, pl. 8, fig. 9.

Globorotalia pseudomiocenica Bolli and Bermudes, 1965, Venezuelan Asoc. Geol. Min. Petr. Bol. Inform., v. 8, p. 140 , pl. 1, fig. 13-15.

Globorotalia pseudopima Blow = Globorotalia $($ Turborotalia) acostaensis pseudopima Blow, 1969. Internatt. Conf. Plankt. Microfoss. Proc., v. 1, p. 387 , pl. 35, fig. 1-7.

Globorotalia scitula (Brady) = Pulvinulina scitula Brady, 1882. Roy. Soc. Edinburgh Proc., v. 11 (1880-1882), p. $716=$ Pulvinulina scitula Banner and Blow, 1961. Cushman Found. Foram. Res. Contrib., v. 11, p. 27, pl. 5, fig. 5.

Globorotalia tosaensis Takayanagi and Saito, 1962. Tohoku Univ., Sci. Rept. Geol., v. 5, p. 81, pl. 28, fig. 11, 12.

Globorotalia truncatulinoides (d'Orbigny) = Rotalina truncatulinoides d'Orbigny, 1839. In Barker-Webb et Berthelot, Hist. Natl. Iles Canaries, "Foraminiferes," v. 2, p. 132, pl. 2, fig. 25-27.

Orbulina suturalis Bronnimann, 1951. Cushman Found. Foram. Res. Contrib., v. 2, p. 135, fig. (1-15); fig. (3-8, 11, $13-16,18,20-22)$; fig. (2-4, 7-12, 15-16, 19-22); fig. 5 .

Orbulina universa d'Orbigny, 1839. In de la Sagra, Foraminiferes: Hist. Phys. Pol. Natl. Cuba, p. 3, pl. 1, fig. 1.

Praeorbulina glomerosa circularis $(\mathrm{Blow})=$ Globigerinoides glomerosa circularis Blow, 1956. Micropaleontology, v. 2, p. 65 , fig. 2 (3-4).

Pulleniatina obliquiloculata (Parker and Jones) $=$ Pullenia obliquiloculata Parker and Jones, 1862. In Carpenter, W. B., et al., Introduction to the Study of the foraminiferes $\mathrm{p}$. 183 = Pullenia sphaeroides (d'Orbigny) var. obliquiloculata Parker and Jones, 1865. Roy. Soc. London Phil. Trans., v. 155 , p. 365,368 , pl. 19 , fig. 4.

Pulleniatina obliquiloculata praecursor Banner and Blow, 1967. Micropaleontology, v. 13, p. 139, pl. 3, fig. 3.

Pulleniatina primalis Banner and Blow, 1967. Micropaleontology, v. 13 , p. 142 , pl. 1 , fig. $3-8$; pl. 3 , fig. 2 .

Sphaeroidinella dehiscens (Parker and Jones) = Spheroidina bulloides d'Orbigny var. dehiscens Parker and Jones, 1865. Roy. Soc. London Phil. Trans., v. 155, p. 369, pl. 19, fig. 5.

Sphaeroidinella seminulina (Schwager) $=$ Globigerina seminulina Schwager, 1866. Novara Expedition, 1857-1859, Geol., v. 2, p. 256 , pl. 7, fig. 112.

Sphaeroidinella subdehiscens Blow = Sphaeroidinella dehiscens subdehiscens Blow, 2959. Am. Paleontol., Bull. v. 39, p. 195, pl. 12, fig. 71,72 .

\section{REFERENCES}

Adegoke, O.S., Dessauvagie,'T. F. J., and Kogbe, C. A., 1971. Planktonic foraminifera in Gulf of Guinea sediments: Micropaleontology, v. 17, p. 197.

Bandy, O. L., 1967. Foraminiferal definition of the boundaries of the Pleistocene in Southern California, U.S.A.: Progress in Oceanogr., v. 4, p. 27.

1969. Relationships of Neogene planktonic foraminifera to paleoocenaography and correlation. In Brönnimann, P. and Renz, H. H. (Eds.), Internatl Conf. Plankt. Microfoss. Proc., Geneva, 1967: Leiden (E. J. Brill).

Bandy, O. L. and Ingle, J. C., 1970. Neogene planktonic events and radiometric scale, California: Geol. Soc. Am. Spec. Paper 124, p. 131

Bandy, O. L. and Wade, M. E., 1967. Miocene-PliocenePleistocene boundaries in deep-water environments: Progress Oceanogr., v. 4, p. 51.
Bandy, O. L. and Wilcoxon, J. A., 1970. The PliocenePleistocene boundary, Italy and California: Geol. Soc. Am. Bull., v. 81 , p. 2939.

Banay, O. L., Casey, R. E., and Wright, R. C., 1971. Late Neogene planktonic zonation, magnetic reversals and radiometric dates, Antarctic to Tropics: Antarctic Res. Ser., vol. 15, Biol. Antarctic Seas IV, Am. Geophys. Union, p. 1-26.

Banner, F. T. and Blow, W. H., 1965. Progress in the planktonic foraminiferal biostratigraphy of the Neogene: Nature, v. 208, p. 1164.

Baumann, P., 1972. Les faunes de foraminiferes de l'Eocene Supérieur a la base du Miocène dans le bassin de Pasir Sud de Kalimantan (Borneo): Rev. d'Inst. Francais Pétrole Ann., Combustibles Liquides, v. 27, p. 817.

Bé, A. W. H. and McIntyre, A., 1970. Globorotalia menardii flexuosa (Koch): An "extinct" foraminiferal subspecies living in the northern Indian Ocean. Deep-Sea Res., v. 17, p. 595 .

Beckman, J. P., 1971. The foraminifera of Sites 68 to 75. In Tracey, J. I., Sutton, G. H., et al., Initial Reports of the Deep Sea Drilling Project, Volume 8: Washington (U.S. Government Printing Office), p. 713.

Berggren, W. A., 1969. Rates of evolution in some Cenozoic planktonic foraminifera: Micropaleontology, v. 15, p. 351 1972. A Cenozoic time-scale-some implications for regional geology and paleobiography: Lethaia, v. 5, p. 195.

Berggren, W. A., Phillips, J. D., Bertels, A., and Wall, D., 1967. Late Pliocene-Pleistocene stratigraphy in deep-sea cores from the south-central North Atlantic: Nature, v. 216 , p. 253.

Bermúdez, P. J. and Bolli, H. M., 1969. Consideraciones sobre los sedimentos del Mioceno medio al reciente de las costas central y oriental de venezuela, $3^{\mathrm{a}}$. parte - los foraminíferos planctónicos: Venezuela, Direc. Geol. Bol. v. 10, p. 137.

Blow, W. H., 1969. Late middle Eocene to Recent planktonic foraminiferal biostratigraphy. In Bronnimann, P. and Renz, H. H. (Eds.), Internatl. Conf. Plankt. Microfoss. Proc., Geneva, 1967: Leiden, (E. J. Brill) p. 199.

Bolli, H. M., 1966. Zonations of Cretaceous to Pliocene marine sediments based on planktonic foraminifera: Venezolana Asoc. Geol. Min. Petrol. Bol. Inform., v. 9, p. 1

1970. The foraminifera of sites 23-31, Leg 4. In Bader, R. G., Gerard, R. D., et al., Initial Reports of the Deep Sea Drilling Project, Volume 4: Washington (U.S. Government Printing Office), p. 577.

Bolli, H. M. and Bermudez, P. J., 1965. Zonation based on planktonic foraminifera of middle Miocene to Pliocene warm water sediments: Venezolana Asoc. Geol. Min. Petrol. Bol. Inform., v. 8, p. 121.

Bolli, H. M., Bourdeaux, J. E., Emiliani, C., Hay, W. W., Hurley, R. J., and Jones, J. I., 1968. Biostratigraphy and paleotemperatures of a section cored on the Nicaragua Rise, Caribbean Sea: Geol. Soc. Amm Bull., v. 79, p. 459.

Boltovskoy, E., 1965. Twilight of foraminiferology: J. Paleontol. v. 39 , p. 383.

1968. Living planktonic foraminifera of the Eastern part of the Tropical Atlantic: Rev. Micropaleontol., v. 11, p. 85 .

1969. Tanatocenosis de Foraminíferos planctónicos en el estrecho de Mozambique: Rev. Españ. Micropaleontol., v. 1, p. 117.

1971. Planktonic foraminiferal assemblages of the epipelagic zone and their thanatocoenosis. In Funnell, B. M. and Riedel, W. R. (Eds.), The micropaleontology of oceans: Cambridge (Cambridge Univ. Press) p. 277.

1973. Estudio de testigos submarinos del Atlantico Sudoccidental. Argentina Museo Cien. Natl. Rev. Geol., v. 7 , p. 215 
Cifelli, R., 1970. Age relationships of Mid-Atlantic Ridge sediments: Geol. Soc. Am. Spec. Paper 124, p. 47.

Ericson, D. B. and Wollin, G., 1964. The deep and the past: New York (Alfred A. Knopf).

1968. Pleistocene climates and chronology in deep-sea sediments: Science, v. 162, p. 1227.

Ericson, D. B., Ewing, M., and Wollin, G., 1964. The Pleistocene epoch in deep-sea sediments: Science, v. 146. p. 723.

Glaessner, M. F. 1937. Planktonforaminiferen aus der Kreide und dem Eozan und ihre stratigraphische Bedeutung:Moscow Univ. Paleontol. Lab. Studies Micropaleontol. v. 1, p. 29.

Hays, J. D., 1969. Aspects of the Quaternary records in deepsea sediments: INQUA, 8 th Cong., Res. Communic., Paris.

Hays, J. D. and Berggren, W. A., 1971. Quaternary boundaries and correlations. In Funnell, B. M. and Riedel, W. R. (Eds.), The micropaleontology of oceans: Cambridge (Cambridge Univ. Press) p. 669.

Hornibrook, N. de B., 1967. New Zealand Tertiary microfossil zonation, correlation and climate. In Tertiary correlations and climatic changes in the Pacific: p. 29.

Jenkins, D. G., 1966. Planktonic foraminiferal datum planes in the Pacific and Trinidad Tertiary: New Zealand J. Geol. Geophys., v. 9, p. 424.

1967. Planktonic foraminiferal zones and new taxa from the lower Miocene to the Pleistocene of New Zealand: New Zealand J. Geol. Geophys., v. 10, p. 1064. 1971. New Zealand Cenozoic planktonic foraminifera: New Zealand Geol. Surv. Paleontol. Bull. v. 42, p. 1.

Jenkins, D. G. and Orr, W. H., 1972. Planktonic foraminiferal biostratigraphy of the eastern Equatorial Pacific DSDP Leg 9. In Hays, J. D. et al., Initial Reports of the Deep Sea Drilling Project, Volume 9: Washington (U.S. Government Printing Office), p. 1059.

Kaneps, A. G., 1973. Cenozoic planktonic foraminifera from the eastern Equatorial Pacific Ocean. In, van Andel, T. H., Heath, G. R., et al., Initial Reports of the Deep Sea Drilling Project, Volume 16: Washington (U.S. Government Printing Office), p. 713.

Kennett, J. P., 1966. The Globorotalia crassaformis bioseries in north Westland and Marlborough, New Zealand: Micropaleontology, v. 12, p. 235.

1968. Latitudinal variation in Globigerina pachyderma (Ehrenberg) in surface sediments of the southwest Pacific Ocean: Micropaleontology, v. 14, p. 305.

Kennett, J. P. and Geitzenauer, K. R., 1969. PliocenePleistocene boundary in a South Pacific deep-sea core: Nature, v. 224 , p. 899.

Kennett, J. P., Watkins, N. D., and Vella, P., 1971. Paleomagnetic chronology of Pliocene-early Pleistocene climates and the Plio-Pleistocene boundary in New Zealand: Science, v. 171, p. 276.

Koch, R., 1923. Die jungtertiare Foraminiferenfauna von Kabu (Res. Surabaja, Java) Eclog. Geol. Helv., v. 18, p. 342.

Krasheninnikov, V. A., 1971. Stratigrafia i foraminifery kainozojskikh pelagicheskikh osadkov severo-zapadnoj chasti Tikhogo okeana: Voprosy Mikropaleontol., p. 140.

Lamb, J. L., 1969. Planktonic foraminiferal datums and late Neogene epoch boundaries in the Mediterranean, Caribbean, and Gulf of Mexico: Gulf Coast Assoc. Geol. Soc. Trans., v. 19 , p. 559.

Lidz, L., 1966. Deep-Sea Pleistocene biostratigraphy: Science, v. 154 , p. 1448.

McInnes, B. A., 1965. Globorotalia miozea Finlay as an ancestor of Globorotalia inflata (d'Orbigny): New Zealand Geol. Geophys., v. 8, p. 104.

Olsson, R. K., 1971. Pliocene-Pleistocene planktonic foraminiferal biostratigraphy of the Northeastern Pacific. In Farinacci, A. (Ed.), Second Plank. Conf. Rome, 1970, Proc. p. 921.

Orr, W. N., 1969. Variation and distribution of Globigerinoides ruber in the Gulf of Mexico: Micropaleontology, v. 15, p. 373.

Parker, F. L., 1967. Late Tertiary biostratigraphy (planktonic foraminifera) of tropical Indo-Pacific deep-sea cores: Am. Paleontol. Bull., v. 52, p. 111. 1973. Late Cenozoic biostratigraphy (planktonic foraminifera) of tropical Atlantic deep-sea sections: Rev. Españ. Micropaleontol. v. 5, p. 253.

Plummer, H. J., 1926. Foraminifera of the Midway Formation in Texas: Texas Univ. Bull. 2644, p. 1.

Poag, C. W., 1971. A reevaluation of the Gulf Coast PliocenePleistocene boundary: Gulf Coast Assoc. Geol. Soc. Trans., v. 21, p. 291.

Poag, C. W. and Akers, W. H., 1967. Globigerina nepenthes Todd of Pliocene age from the Gulf Coast: Cushman Found. Foram. Res. Contrib., v. 18, p. 168.

Postuma, J. A., 1971. Manual of planktonic foraminifera: The Hague (Elsevier).

Reiss, Z. and Gvirtzman, G., 1966. Subsurface Neogene stratigraphy: Internatl. Union Geol. Sci., Comm. Strat., Comm. Mediter. Neog. Strat. 3rd Sess., Berne, Proc., p. 312.

Ruddiman, W. F., Tolderlund, D. S., and Bé, A. W. H., 1970. Foraminiferal evidence of a modern warming of the North Atlantic Ocean. Deep-Sea Res., v. 17, p. 141.

Soediono, H., 1970. Planktonic foraminifera from the Velez Rubio region, S. E. Spain. Part 2: The Espejos formation: Rev. Españ. Micropaleontol, v. 2, p. 215.

Walters, R., 1965. The Globorotalia zelandica and G. miozea lineages: New Zealand J. Geol. Geophys., v. 8, p. 109. 


\section{PLATE 1}

Figure $1 \quad$ Globigerina ampliapertura Bolli, $\times 90$; Sample 25310-2, 40-42 cm, hypotype: slide FMACN 7649.

Figure 2 Globigerina ampliapertura Bolli, $\times 80$; Sample 25311, CC; hypotype: slide FMACN 7649.

Figure 3 Globigerina ampliapertura Bolli, $\times 100$; Sample 253-11, CC; hypotype: slide FMACN 7649.

Figure 4 Candeina nitida d'Orbigny, $\times 64$; Sample 253-1, CC; hypotype: slide FMACN 7650.

Figure $5 \quad$ Globigerina ampliapertura Bolli, $\times 72$; Sample 25311, CC; hypotype: slide FMACN 7649.

Figure $6 \quad$ Globigerina ampliapertura Bolli, $\times 80$; Sample 25311, CC; hypotype: slide FMACN 7649.

Figure $7 \quad$ Globigerina angiroporoides Hornibrook, $\times 100$; Sample 253-11-6, 40-42 cm; hypotype: slide FMACN 7651.

Figure $8 \quad$ Globigerina angiroporoides Hornibrook, $\times 100$; Sample 253-11-6, 40-42 cm; hypotype: slide FMACN 7651.

Figure 9 Globigerina calida Parker, $\times 56$; Sample 251-2-2, $39-41 \mathrm{~cm}$; hypotype: slide FMACN 7652.

Figure 10 Globigerina decoraperta Takayanagi and Saito, $\times 200$; Sample 258A-5-5, 148-150 cm; hypotype: slide FMACN 7653.

Figure $11 \quad$ Globigerina euapertura Jenkins, $\times 100$; Sample 254-19, CC; hypotype: slide FMACN 7654.

Figure 12 Globigerina euapertura Jenkins, $\times 120$; Sample 254-19, CC; hypotype: slide FMACN 7654.

Figure 13 Globigerina juvenilis Bolli, $\times 100$; Sample 253-10, CC; hypotype: slide FMACN 7655.

Figure 14 Globigerina linaperta Finlay, $\times 100$; Sample 250A19, CC; hypotype: slide FMACN 7656.

Figure 15 Globigerina nepenthes Todd, $\times 80$; Sample 254-4-4, 115-117 cm; hypotype: slide FMACN 7657.

Figure 16 Globigerina nepenthes Todd, $\times 80$; Sample 254-4-4, 115-117 cm; hypotype: slide FMACN 7657. 
PLATE 1
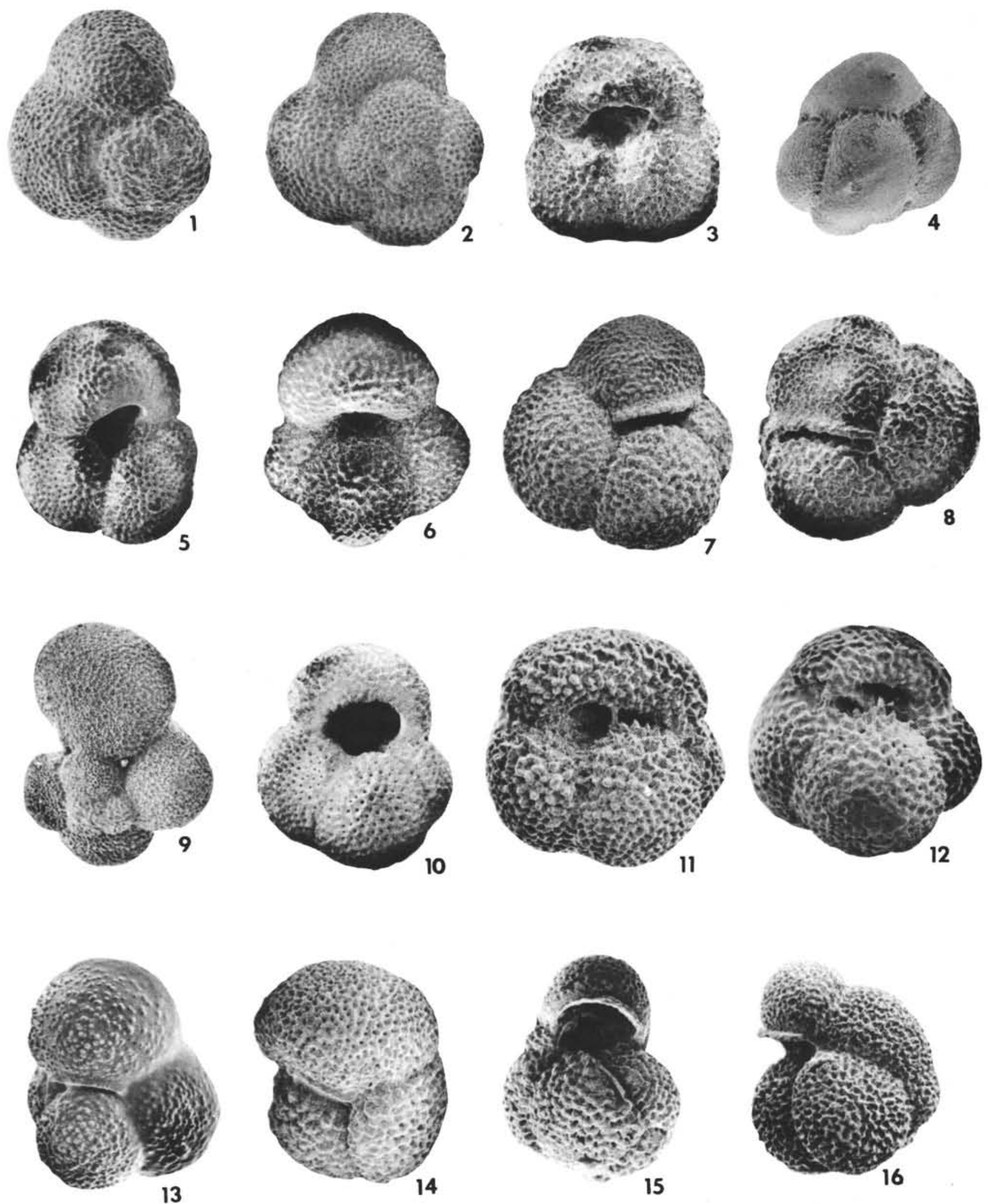


\section{PLATE 2}

Figure 1

Figure 2

Figure 3

Figure 4

Figure 5

Figure 6

Figure 7

Figure 8

Figure 9

Figure 10

Figure 11

Figure 12

Figure 13

Figure 14

Figure 15

Figure 16
Globigerina cryptomphala (Glaessner), $\times 80$; Sample 254-18-2, 40-42 cm; hypotype: slide FMACN 7658.

Globigerina cryptomphala (Glaessner), $\times 60$; Sample 254-17, CC; hypotype: slide FMACN 7658.

Globigerina falconensis Blow, $\times 120$; Sample 254-1, CC; hypotype: slide FMACN 7659.

Globigerina bulloides d'Orbigny, ×56; Sample 2542-1, 115-117 cm; hypotype: slide FMACN 7660.

Globigerina cryptomphala (Glaessner), $\times 72$; Sample 254-19-1, 40-42 cm; hypotype: slide FMACN 7658.

Globigerina cryptomphala (Glaessner), $\times 48$; Sample 254-17, CC; hypotype: slide FMACN 7658.

Globigerina cryptomphala (Glaessner), $\times 48$; Sample 254-17, CC; hypotype: slide FMACN 7658.

Globigerina cryptomphala (Glaessner), $\times 80$; Sample 253-10-2, 40-42 cm; hypotype: slide FMACN 7658.

Globigerina cryptomphala (Glaessner), $\times 80$; Sample 254-5-3, 40-42 cm; hypotype: slide FMACN 7658.

Globigerina cryptomphala (Glaessner), $\times 80$; Sample 254-17, CC; hypotype: slide FMACN 7658.

Globigerina pachyderma (Ehrenberg) (dex.), $\times 120$; Sample 251-3-2, 40-42 cm; hypotype: slide FMACN 7661.

Globigerina pachyderma (Ehrenberg) (dex.), $\times 120$; Sample 25l-3-2, 40-42 cm; hypotype: slide FMACN 7661.

Globigerina "praerubra," $\times 88$; Sample 254-11, CC; hypotype: slide FMACN 7662.

Globigerina "praerubra," $\times 120$; Sample 254-12-2, 40-42 cm; hypotype: slide FMACN 7662.

Globigerina "praerubra," $\times 100$; Sample 254-18-5, 115-117 cm; hypotype: slide FMACN 7662.

Globigerina "praerubra," $\times 72$; Sample 254-15, CC; hypotype: slide FMACN 7662. 
NEOGENE PLANKTONIC FORAMINIFERA

PLATE 2
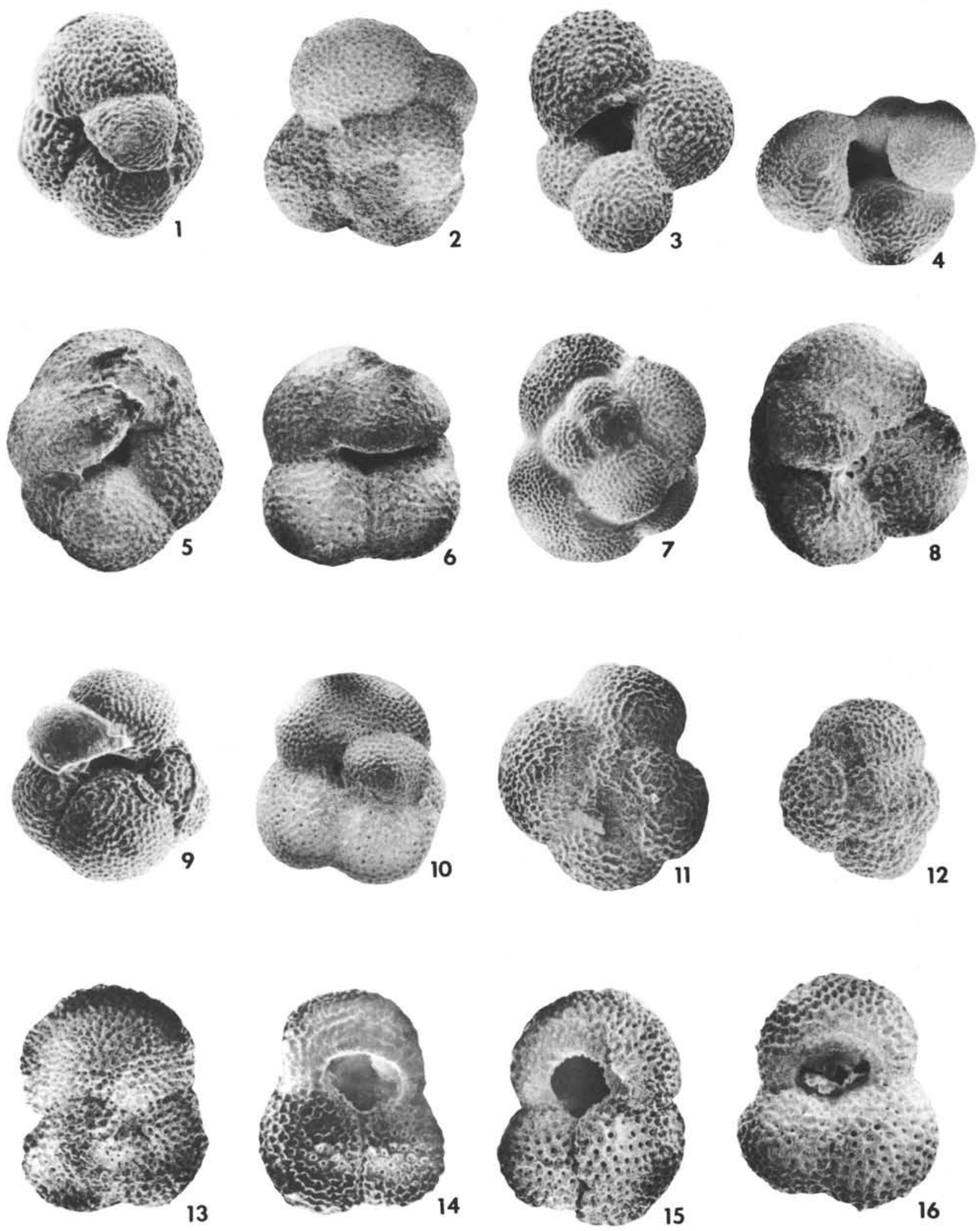
PLATE 3

Figure $1 \quad$ Globigerina quinqueloba Natland, $\times 200$; Sample 251A-1, CC; hypotype: slide FMACN 7663.

Figure 2 Globigerina quinqueloba Natlant, $\times 200$; Sample 251A-1, CC; hypotype: slide FMACN 7663.

Figure $3 \quad$ Globigerinella aequilateralis (Brady), $\times 64$; Sample 2582-1, 88-90 cm; hypotype: slide FMACN 7664.

Figure $4 \quad$ Globigerinita glutinata $($ Egger) $\times 100$; Sample 2504, CC; hypotype: slide FMACN 7665.

Figure $5 \quad$ Globigerina tripartita Koch, $\times 72$; Sample 253-12, CC; hypotype: slide FMACN 7666.

Figure $6 \quad$ Globigerina tripartita Koch, $\times 52$; Sample 253-105, 40-42 cm; hypotype: slide FMACN 7666.

Figure 7

Globigerina tripartita Koch, $\times 60$; Sample 253-12, CC; hypotype: slide FMACN 7666.

Figure $8 \quad$ Globigerina tripartita Koch, $\times 64$; Sample 253-124, 53-55 cm; hypotype: slide FMACN 7666.

Figure 9 Globigerina tripartita Koch, $\times 64$; Sample 253-12, CC; hypotype: slide FMACN 7666.

Figure $10 \quad$ Globigerina tripartita Koch, $\times 60$; Sample 253-10, CC; hypotype: slide FMACN 7666.

Figure $11 \quad$ Globigerina yeguaensis Weinzierl and Applin, $\times 80$; Sample 253-12-4, 53-55 cm; hypotype: slide FMACN 7667.

Figure 12 Globigerina yeguaensis Weinzierl and Applin, $\times 56$; Sample 253-8, CC; hypotype: slide FMACN 7667.

Figure 13 Globigerinita cf. africana Blow and Banner, $\times 56$; Sample 254-19-3, 115-117 cm; hypotype: slide FMACN 7668.

Figure $14 \quad$ Globigerinita $\mathrm{cf}$ africana Blow and Banner, $\times 48$; Sample 25418-3, 125-127 cm; hypotype: slide FMACN 7668.

Figure $15 \quad$ Globigerinita cf. africana Blow and Banner, $\times 48$; Sample 254-18-3, 125-127 cm; hypotype: slide FMACN 7668.

Figure $16 \quad$ Globigerinita cf. africana Blow and Banner, $\times 80$; Sample 254-18-3, 125-127 cm; hypotype: slide FMACN 7668. 
NEOGENE PLANKTONIC FORAMINIFERA

PLATE 3
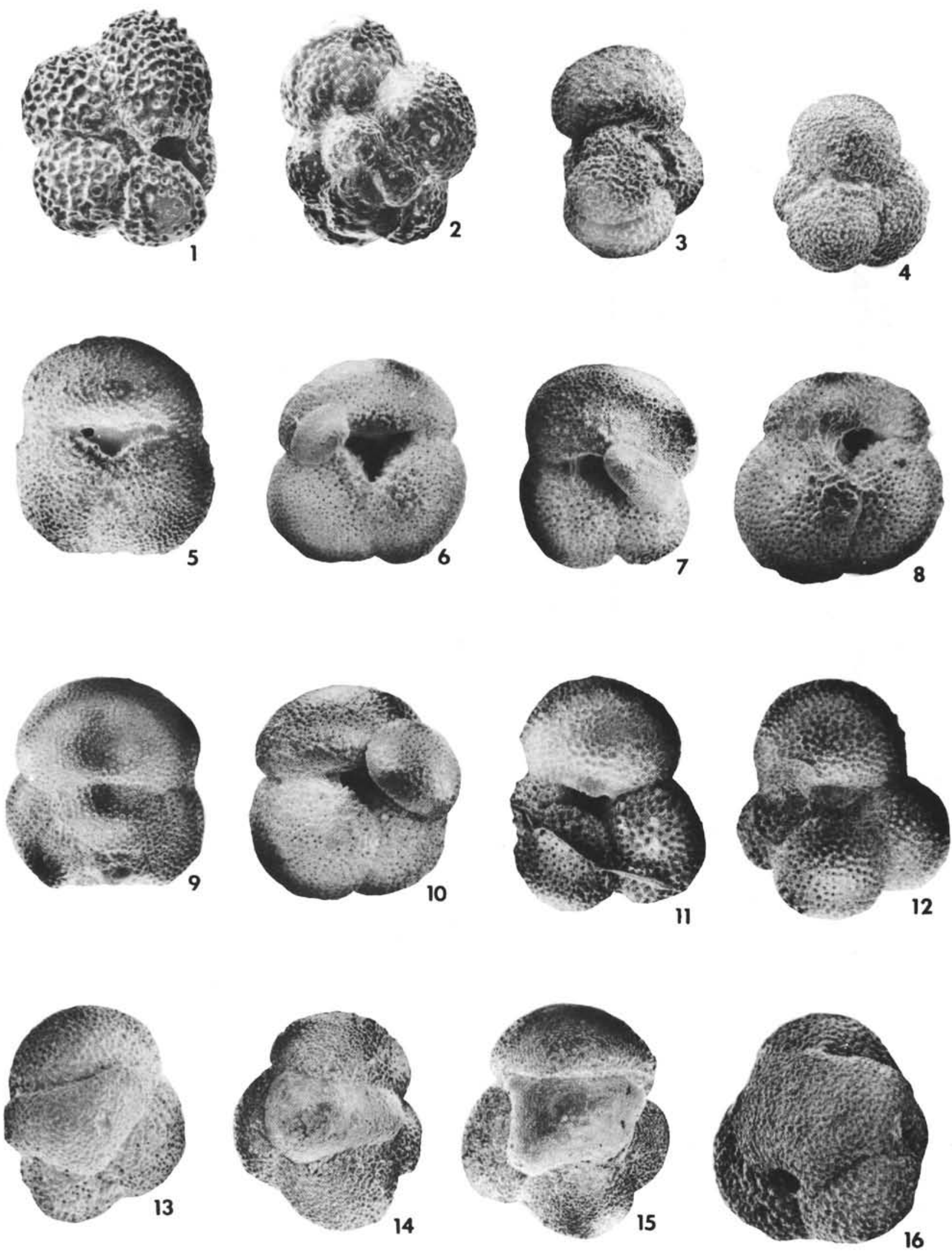


\section{PLATE 4}

Figure $1 \quad$ Globigerinita dissimilis ciperoensis Blow and Banner, $\times 64$; Sample 254-19-1, 40-42 cm; hypotype: slide FMACN 7669.

Figure 2 Globigerina rubescens Hofker, $\times 160$; Sample 2581-4, 20-22 cm; hypotype: slide FMACN 7670.

Figure 3 Globigerinoides bulloideus Crescenti, $\times 80$; Sample 254-10-2, 115-117 cm; hypotype: slide FMACN 7671 .

Figure 4 Globigerinoides bulloideus Crescenti, $\times 80$; Sample 254-10-2, 115-117 cm; hypotype: slide FMACN 7671 .

Figure $5 \quad$ Globigerinita dissimilis ciperoensis Blow and Banner, $\times 52$; Sample 254-19-1, 40-42 cm; hypotype: slide FMACN 7669.

Figure $6 \quad$ Globigerinita dissimilis dissimilis (Cushman and Bermúdez), $\times 72$; Sample 254-19, CC; hypotype: slide FMACN 7672.

Figure 7 Globigerinita unicava (Bolli, Loeblich, and Tappan), $\times 80$; Sample 254-5-3, 40-42 cm; hypotype: slide FMACN 7673.

Figure 8 Globigerinita unicava (Bolli, Loeblich, and Tappan), $\times 80$; Sample 254-5-3, 40-42 cm; hypotype: slide FMACN 7673.

Figure 9 Globigerinita unicava (Bolli, Loeblich, and Tappan), $\times 88$; Sample $254-5-3,40-42 \mathrm{~cm}$; hypotype: slide FMACN 7673.

Figure $10 \quad$ Globigerinoides amplus Perconig, $\times 64$; Sample 253-5-6, 40-42 cm; hypotype: slide FMACN 7674.

Figure $11 \quad$ Globigerinoides amplus Perconig, $\times 80$; Sample 253-5-6, 40-42 cm; hypotype: slide FMACN 7674.

Figure 12 Globigerinoides amplus Perconig, $\times 56$; Sample 253-6-5, 40-42 cm; hypotype: slide FMACN 7674.

Figure $13 \quad$ Globigerinoides amplus Perconig, $\times 76$; Sample 253-5-6, 40-42 cm; hypotype: slide FMACN 7674.

Figure $14 \quad$ Globigerinoides amplus Perconig, $\times 68$; Sample 253-4-1, 45-47 cm; hypotype: slide FMACN 7674.

Figure $15 \quad$ Globigerinoides amplus Perconig, $\times 60$; Sample 253-6-5, 40-42 cm; hypotype: slide FMACN 7674.

Figure 16 Globigerinoides conglobatus (Brady, $\times 48$; Sample 254-1-2, 115-117 cm; hypotype: slide FMACN 7674. 
PLATE 4
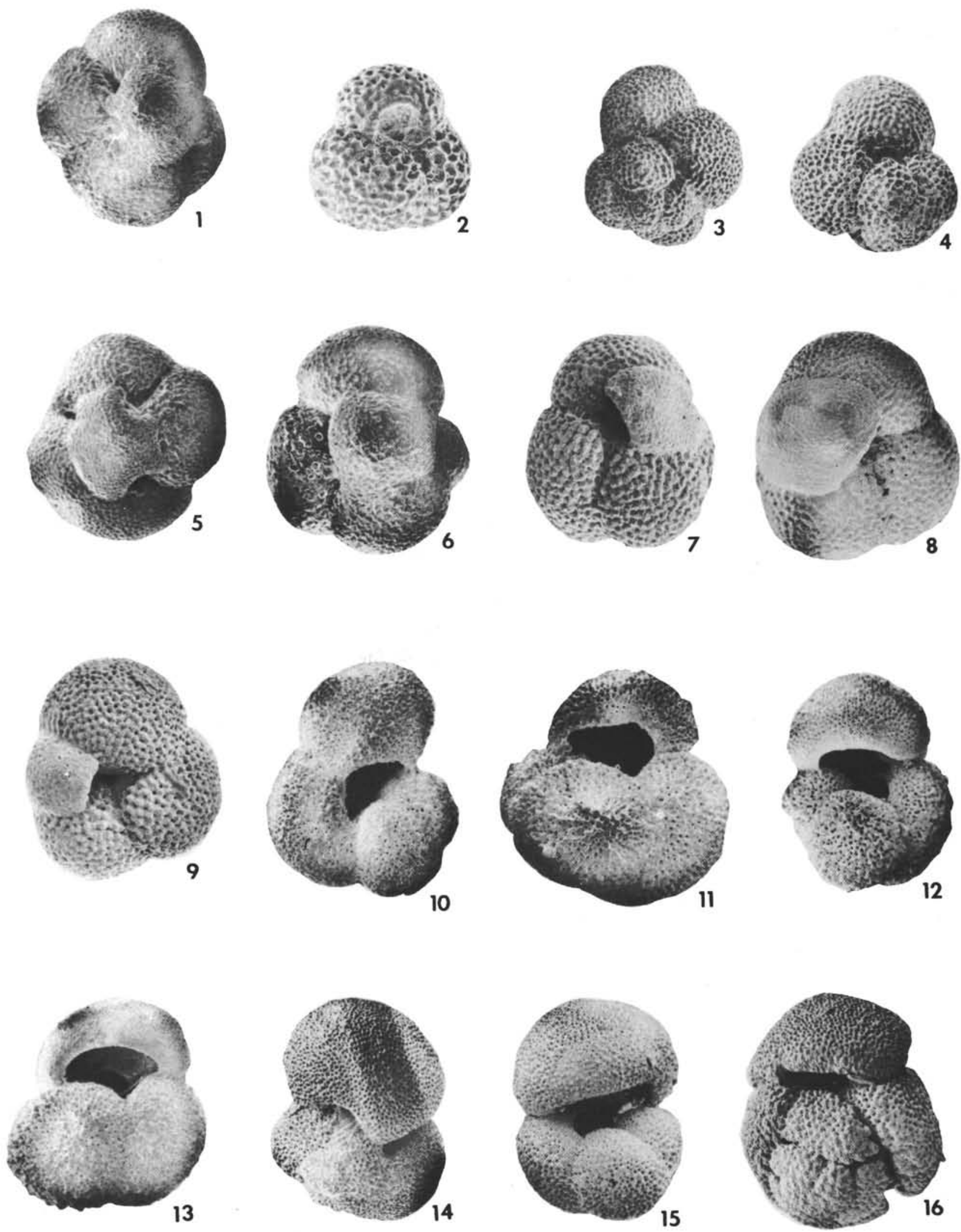


\section{PLATE 5}

Figure 1

Figure 2

Figure 3

Figure 4

Figure 5

Figure 6

Figure 7

Figure 8

Figure 9

Figure 10

Figure 11

Figure 12

Figure 13

Figure 14

Figure 15

Figure 16
Globigerinoides mitra Todd, $\times 52$; Sample 253-7-1, 70-72 cm; hypotype: slide FMACN 7676.

Globigerinoides mitra Todd, $\times 80$; Sample 253-7, CC; hypotype: slide FMACN 7676.

Globigerinoides elongatus (d'Orbigny), $\times 80$; Sample 254-1, CC; hypotype: slide FMACN 7677.

Globigerinoides obliquus Bolli, s.l. $\times 80$; Sample 254-2-3, 40-42 cm; hypotype: slide FMACN 7678.

Globigerinoides obliquus Bolli, s.l. $\times 64$; Sample 254-2-3, 40-42 cm; hypotype: slide FMACN 7678.

Globigerinoides obliquus Bolli, s.l. $\times 64$; Sample 253-3-4, 40-42 cm; hypotype: slide FMACN 7678.

Globigerinoides pyramidalis (van den Broeck), $\times 64$; Sample 253-1-3, $48-50 \mathrm{~cm}$; hypotype: slide FMACN 7679.

Globigerinoides sicanus de Stefani, $\times 100$; Sample 253-8-2, 40-42 cm; hypotype: slide FMACN 7680.

Globigerinoides sicanus de Stefani, $\times 100$; Sample 253-8-4, 60-62 cm; hypotype: slide FMACN 7680.

Globigerinoides sicanus de Stefani, $\times 80$; Sample 254-10-6, 40-42 cm; hypotype: slide FMACN 7680 .

Globigerinoides sicanus de Stefani, $\times 100$; Sample 254-12-4, 140-142 cm; hypotype: slide FMACN 7680 .

Globigerinoides sicanus de Stefani, $\times 80$; Sample 254-12-4, 140-142 cm; hypotype: slide FMACN 7680 .

Globigerinoides trilobus (Reuss), s.l. $\times 48$; Sample 253-2-1, 100-102 cm; hypotype: slide FMACN 7681 .

Globigerinoides trilobus (Reuss), s.l. $\times 48$; Sample 253-2-4, 40-42 cm; hypotype: slide FMACN 7681.

Globigerinoides trilobus (Reuss), s.l. $\times 60$; Sample 253-2-1, 100-102 cm; hypotype: slide FMACN 7681 .

Globigerinoides fistulosus (Schubert), $\times 32$; Sample 253-2-1, 50-52 cm; hypotype: slide FMACN 7682. 
NEOGENE PLANKTONIC FORAMINIFERA

PLATE 5
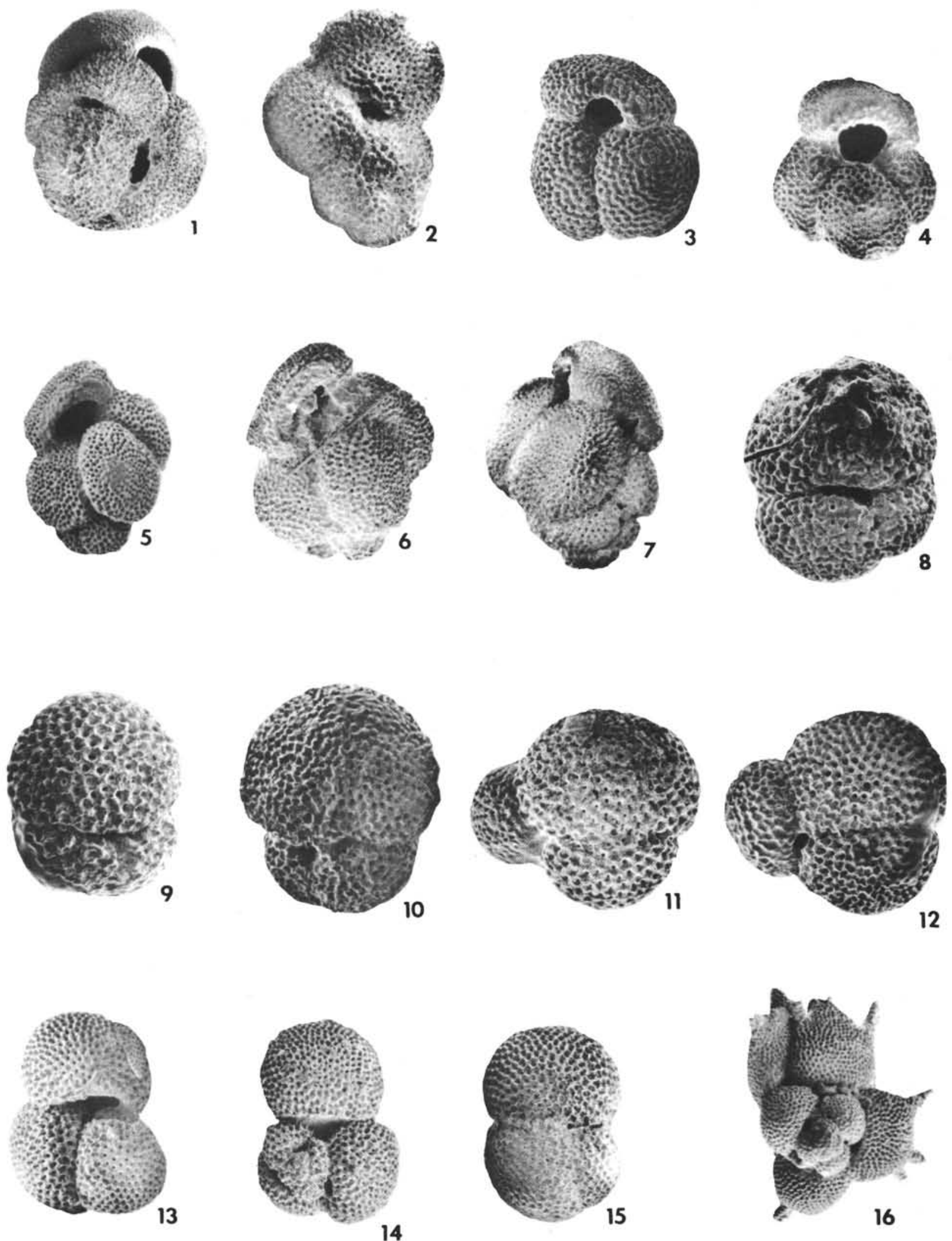


\section{PLATE 6}

Figure $1 \quad$ Globigerinoides trilobus (Reuss), $f$. sacculifera (Brady), $\times 32$; Sample 253-2-6, 40-42 cm; hypotype: slide FMACN 7683.

Figure 2 Globigerinoides trilobus (Reuss), $f$. sacculifera (Brady) $\times 40$; Sample 253-2-6, 40-42; hypotype: slide FMACN 7683.

Figure 3 Globoquadrina hexagona (Natland), $\times 100$; Sample 251-5, CC; hypotype: slide FMACN 7684.

Figure 4 Globigerinoides tenellus Parker, $\times 120$; Sample 258A-1-6, 110-112 cm; hypotype: slide FMACN 7685 .

Figure $5 \quad$ Globigerinopsis aguasayensis Bolli, $\times 48$; Sample 254-6-6, 115-117 cm; hypotype: slide FMACN 7686 .

Figure $6 \quad$ Globigerinopsis aguasayensis Bolli, $\times 48$; Sample 254-6, CC; hypotype: slide FMACN 7686.

Figure $7 \quad$ Globigerinopsis aguasayensis Bolli, $\times 48$; Sample 254-6, CC; hypotype: slide FMACN 7686.

Figure $8 \quad$ Globigerinopsis aguasayensis Bolli, $\times 40$; Sample 254-6-5, 40-42 cm; hypotype: slide FMACN 7686.

Figure $9 \quad$ Globigerinopsis aguasayensis Bolli, $\times 40$; Sample 254-7-2, 115-117 cm; hypotype: slide FMACN 7686 .

Figure $10 \quad$ Globigerinopsis aguasayensis Bolli, $\times 40$; Sample 254-7-5, 115-117 cm; hypotype: slide FMACN 7686.

Figure $11 \quad$ Globigerinopsis aguasayensis Bolli, $\times 52$; Sample 254-5-3, 115-117 cm; hypotype: slide FMACN 7686.

Figure 12 Globigerinopsis aguasayensis Bolli, $\times 32$; Sample 254-6, CC; hypotype: slide FMACN 7686.

Figure $13 \quad$ Globigerinopsis aguasayensis Bolli, $\times 40$; Sample 254-6-5, 40-42 cm; hypotype slide FMACN 7686.

Figure $14 \quad$ Globigerinopsis aguasayensis Bolli, $\times 40$; Sample 254-7, CC; hypotype: slide FMACN 7686.

Figure 15 Globoquadrina altispira (Cushman and Jarvis), $\times 56$; Sample $251 \mathrm{~A}-7-5,40-42 \mathrm{~cm}$; hypotype: slide FMACN 7687.

Figure 16 Globoquadrina altispira (Cushman and Jarvis), $\times 48$; Sample 251 A-13-1, 66-68 cm; hypotype: slide FMACN 7687. 

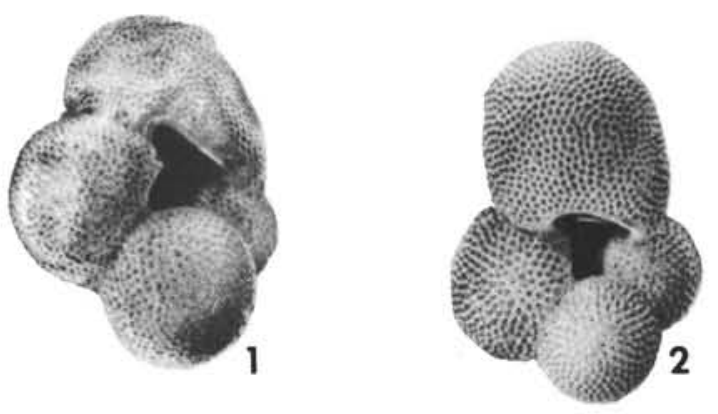

PLATE 6
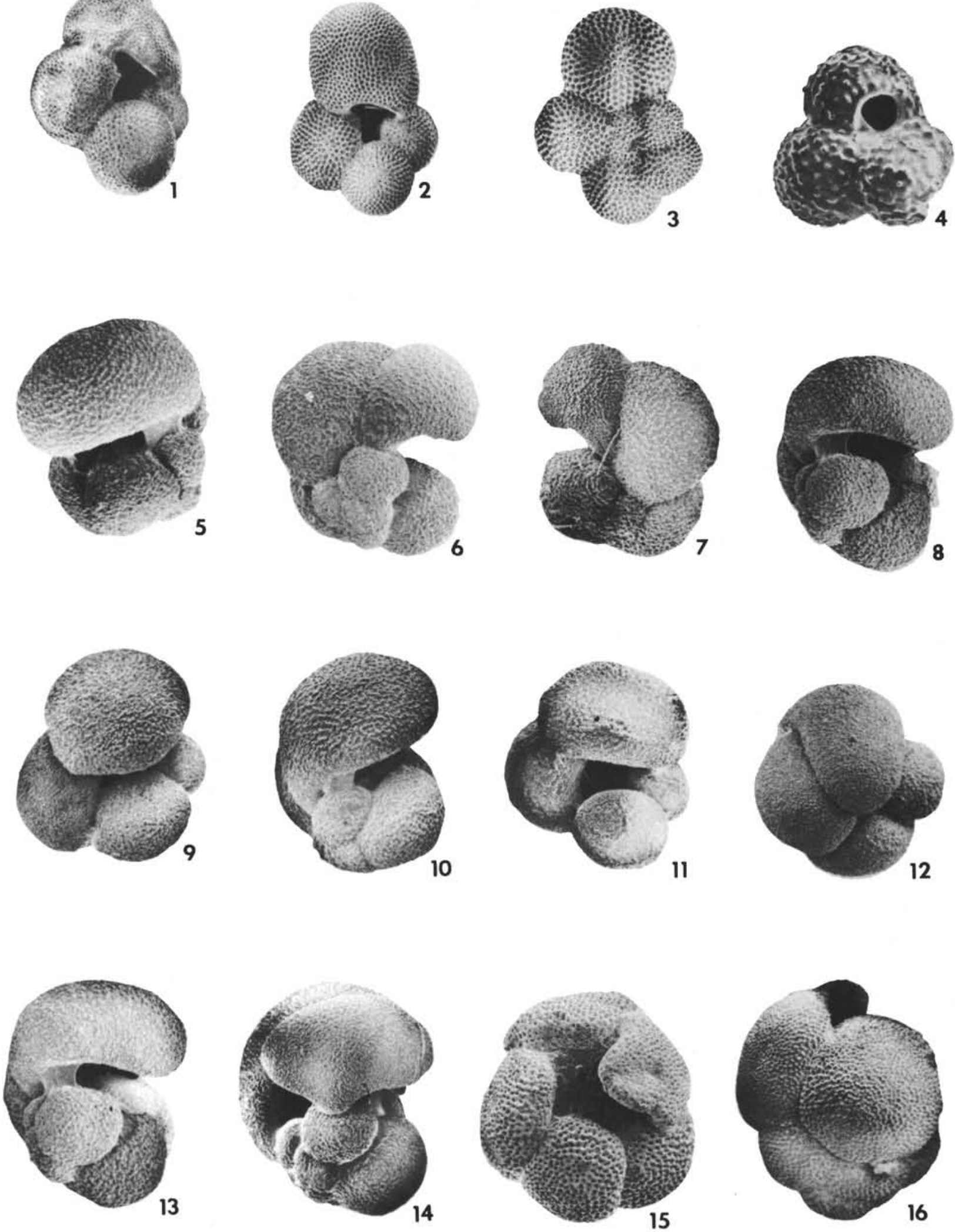


\section{PLATE 7}

Figure 1

Figure 2

Figure 3

Figure 4

Figure 5

Figure 6

Figure 7

Figure 8

Figure 9

Figure 10

Figure 11

Figure 12

Figure 13

Figure 14

Figure 15

Figure 16
Globoquadrina altispira (Cushman and Jarvis), $\times 60$; Sample 253-4-6, 40-42 cm; hypotype: slide FMACN 7687.

Globoquadrina dehiscens dehiscens (Chapman, Parr, and Collins), $\times 64$; Sample 253-7-1, 70-72 cm; hypotype: slide FMACN 7688.

Globoquadrina dehiscens dehiscens (Chapman, Parr, and Collins), $\times 64$; Sample 253-7-1, 70-72 cm; hypotype: slide FMACN 7688.

Globoquadrina dehiscens dehiscens (Chapman, Parr, and Collins), $\times 60$; Sample 254-10-5, 40-42 cm; hypotype: slide FMACN 7688.

Globoquadrina dehiscens praedehiscens Blow and Banner, $\times 48$; Sample 254-19-1, 40-42 cm; hypotype: slide FMACN 7689.

Globoquadrina dehiscens praedehiscens Blow and Banner, $\times 56$; Sample 254-18-6, $115-117 \mathrm{~cm}$; hypotype: slide FMACN 7689.

Globoquadrina dehiscens praedehiscens Blow and Banner, $\times 48$; Sample 254-19-5, $115-117 \mathrm{~cm}$; hypotype: slide FMACN 7689.

Globoquadrina dehiscens praedehiscens Blow and Banner, $\times 48$; Sample 254-19-2, $115-117 \mathrm{~cm}$; hypotype: slide FMACN 7689.

Globoquadrina dehiscens praedehiscens Blow and Banner, $\times 60$; Sample 253-9-5, 40-42 cm; hypotype: slide FMACN 7689.

Globoquadrina dutertrei (d'Orbigny), ×64; Sample 258A-I-1, 61-63 cm; hypotype: slide FMACN 7690 .

Globoquadrina venezuelana (Hedberg), $\times 80$; Sample 255-3, CC; hypotype: slide FMACN 7691.

Globoquadrina venezuelana (Hedberg), $\times 56$; Sample 255-4-2, 39-41 cm; hypotype: slide FMACN 7691.

Globoquadrina venezuelana (Hedberg), $\times 60$; Sample 251A-16-3, 40-42 cm; hypotype: slide FMACN 7691.

Globoquadrina venezuelana (Hedberg), $\times 68$; Sample 254-2-3, 115-117 cm; hypotype: slide FMACN 7691.

Globoquadrina humerosa (Takayanagi and Saito), $\times 100$; Sample 251-8-1, 40-42 cm; hypotype: slide FMACN 7692.

Globorotalia cf. humilis (Brady), $\times 240$; Sample 250-3-1, 39-43 cm; hypotype: is lost. 
PLATE 7
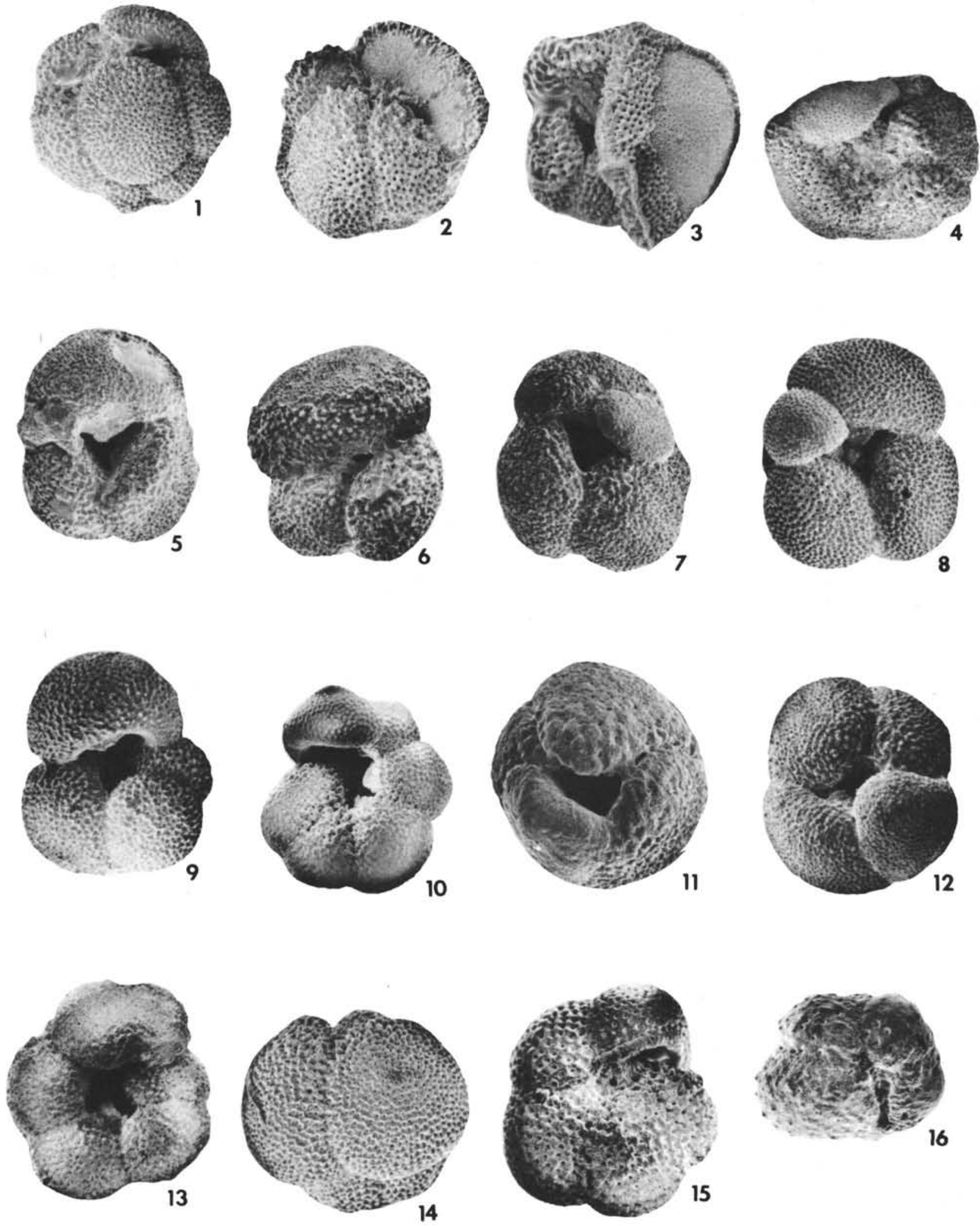
E. BOLTOVSKOY

\section{PLATE 8}

Figure 1 Globorotalia acostaensis Blow, $\times 120$; Sample 251A-6-4, 40-42 cm; hypotype: slide FMACN 7693.

Figure 2 Globorotalia acostaensis Blow, $\times 100$; Sample 251A-6-4, 40-42 cm; hypotype: slide FMACN 7693.

Figure 3 Globorotalia acostaensis Blow, $\times 100$; Sample 251A-5-3, 40-42 cm; hypotype: slide FMACN 7693.

Figure $4 \quad$ Globorotalia acostaensis Blow, $\times 80$; Sample 251A6-5, 125-127 cm; hypotype: slide FMACN 7693.

Figure 5 Globorotalia crassaformis (Galloway and Wissler), s.l.. $\times 60$; Sample 254-2-6, 40-42 cm; hypotype: slide FMACN 7694.

Figure 6 Globorotalia crassaformis (Galloway and Wissler), s.l., $\times 60$; Sample 254-2-6, 40-42 cm; hypotype: slide FMACN 7694.

Figure $7 \quad$ Globorotalia crassaformis (Galloway and Wissler), s.l., $\times 64$; Sample 254-2-6, 40-42 cm; hypotype: slide FMACN 7694.

Figure 8 Globorotalia crassaformis (Galloway and Wissler), s.l., $\times 60$; Sample 254-2-6, 40-42 cm; hypotype: slide FMACN 7694.

Figure 9 Globorotalia crassaformis (Galloway and Wissler), s.l., $\times 56$; Sample 253-1, CC; hypotype: slide FMACN 7694.

Figure 10 Globorotalia crassaformis (Galloway and Wissler), s.l., $\times 56$; Sample 254-1-3, 100-102 cm; hypotype: slide FMACN 7694.

Figure 11 Globorotalia crassaformis (Galloway and Wissler), s.l., $\times 48$; Sample 258-3-4, 40-42 cm; hypotype: slide FMACN 7694.

Figure 12 Globorotalia crassula Cushman and Stewart, $\times 60$; Sample 258A-6-4, 104-106 cm; hypotype: slide FMACN 7695.

Figure $13 \quad$ Globorotalia crassula Cushman and Stewart, $\times 52$; Sample 258A-4-2, 75-77 cm; hypotype: slide FMACN 7695.

Figure $14 \quad$ Globorotalia crassula Cushman and Stewart, $\times 72$; Sample 258A-4-1, 120-122 cm; hypotype: slide FMACN 7695.

Figure $15 \quad$ Globorotalia crassula Cushman and Stewart, $\times 52$; Sample 258A-5-2, 60-62 cm; hypotype: slide FMACN 7695.

Figure $16 \quad$ Globorotalia crassula Cushman and Stewart, $\times 60$; Sample 258-3-4, 40-42 cm; hypotype: slide FMACN 7695.

Figure $17 \quad$ Globorotalia crassula Cushman and Stewart, $\times 52$; Sample 258A-4-2, 105-107 cm; hypotype: slide FMACN 7695. 
PLATE 8
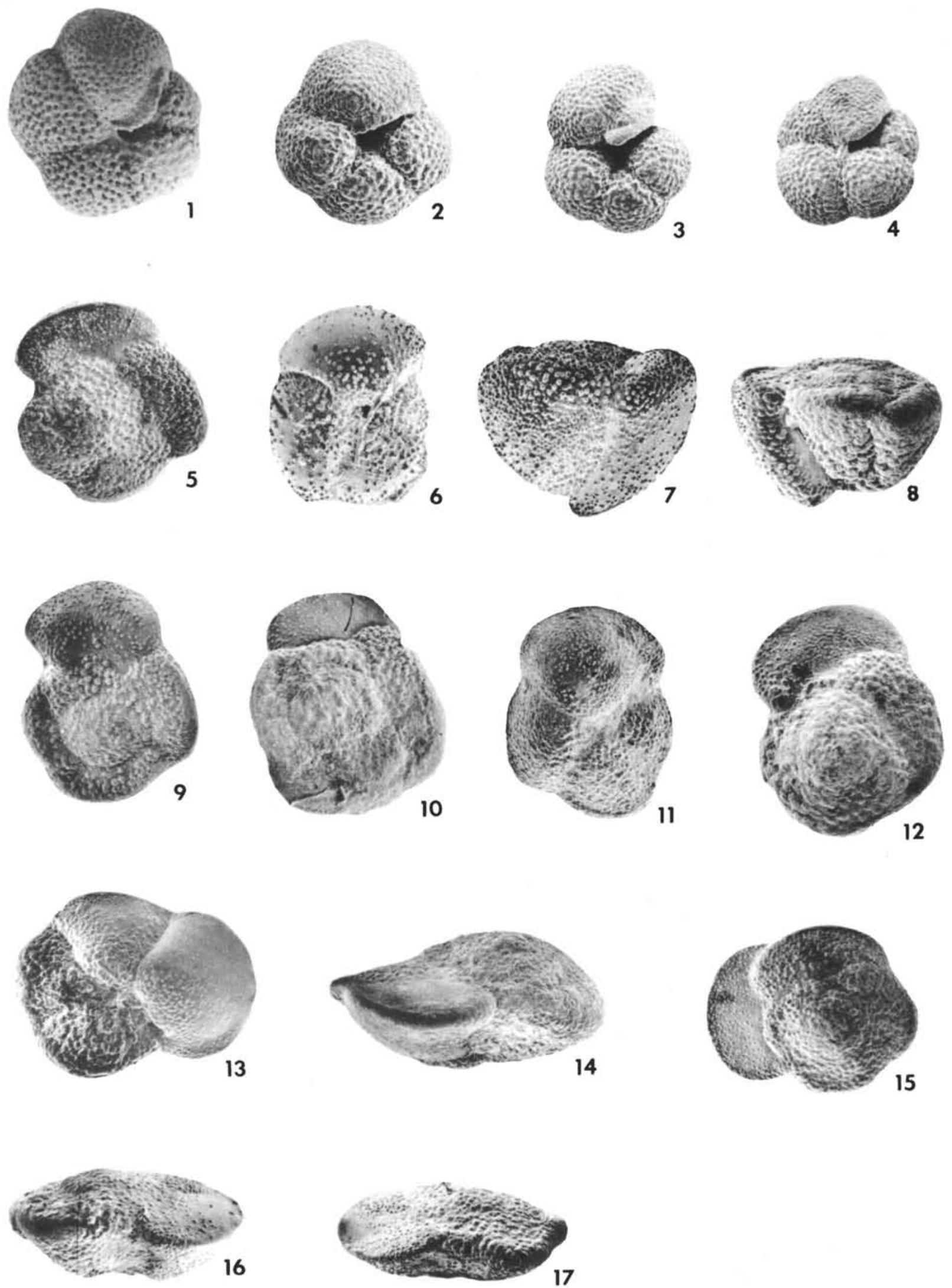


\section{PLATE 9}

Figure 1

Figure 2

Figure 3

Figure 4

Figure 5

Figure 6

Figure 7

Figure 8

Figure 9

Figure 10

Figure 11

Figure 12

Figure 13

Figure 14

Figure 15

Figure 16
Globorotalia inflata (d'Orbigny), $\times 80$; Sample 2543-4, 115-117 cm; hypotype: slide FMACN 7696.

Globorotalia inflata (d'Orbigny), $\times 56$; Sample 2543-6, 115-117 cm; hypotype: slide FMACN 7696.

Globorotalia inflata (d'Orbigny), $\times 64$; Sample 2542-3, 40-42 cm; hypotype: slide FMACN 7696.

Globorotalia inflata (d'Orbigny), $\times 72$; Sample 2543-1, 40-42 cm; hypotype: slide FMACN 7696.

Globorotalia inflata (d'Orbigny), $\times 64$; Sample 254 3, CC; hypotype: slide FMACN 7696.

Globorotalia inflata (d'Orbigny), $\times 56$; Sample 254 1-3, 100-102 cm; hypotype: slide FMACN 7696.

Globorotalia inflata (d'Orbigny), $\times 64$; Sample 2541-3, 100-102 cm; hypotype: slide FMACN 7696.

Globorotalia inflata (d'Orbigny), $\times 64$; Sample 2542-3, 40-42 cm; hypotype: slide FMACN 7696.

Globorotalia kugleri Bolli, $\times 120$; Sample 253-8, CC; hypotype: slide FMACN 7697.

Globorotalia kugleri Bolli, $\times 120$; Sample 253-8, CC; hypotype: slide FMACN 7697.

Globorotalia menardii (d'Orbigny) (sin.), s.l., $\times 28$; Sample 253-1-6, 48-50 cm; hypotype: slide FMACN 7698.

Globorotalia menardii (d'Orbigny), $f$. flexuosa (Koch), $\times 40$; Sample 253-1-1, 118-120 cm; hypotype: slide FMACN 7700.

Globorotalia margaritae Bolli and Bermúdez, $\times 60$; Sample 253-2-5, 40-42 cm; hypotype: slide FMACN 7701.

Globorotalia margaritae Bolli and Bermúdez, $\times 60$; Sample 253-2, CC; hypotype: slide FMACN 7701.

Globorotalia margaritae Bolli and Bermúdez, $\times 60$; Sample 253-2-5, 40-42 cm; hypotype: slide FMACN 7701.

Globorotalia margaritae Bolli and Bermúdez, X80; Sample 253-2, CC; hypotype: slide FMACN 7701 . 
PLATE 9
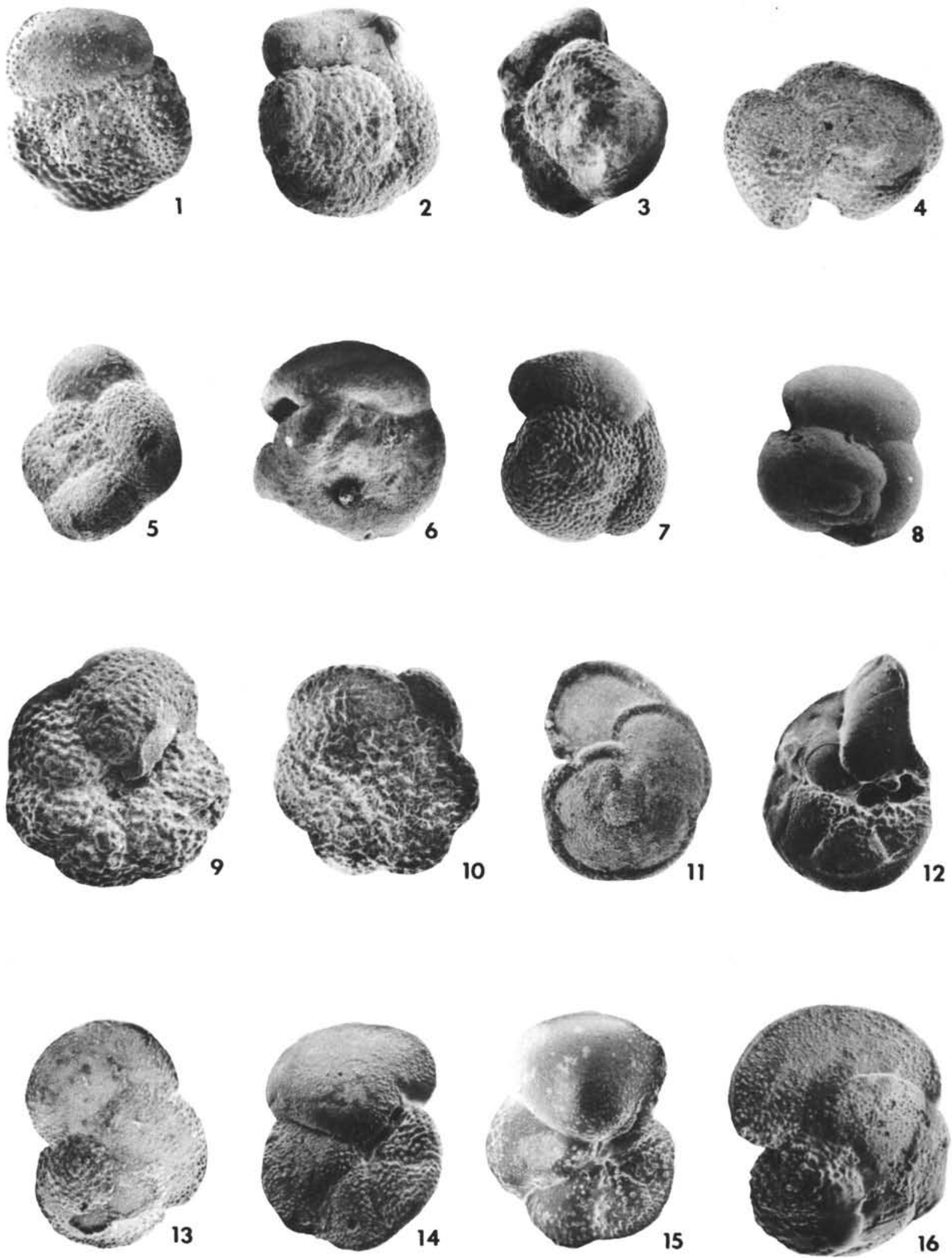
Figure 1 Globorotalia crotonensis Conato and Follador, $\times 40$; Sample 251A-1-5, 40-42 cm; hypotype: slide FMACN 7702.

Figure 2 Globorotalia crotonensis Conato and Follador, $\times 48$; Sample 251A-4, CC; hypotype: slide FMACN 7702.

Figure 3 Globorotalia crotonensis Conato and Follador, $\times 80$; Sample 251A-4, CC; hypotype: slide FMACN 7702.

Figure 4 Globorotalia crotonensis Conato and Follador, $\times 64$; Sample 251-10-5, 40-42 cm; hypotype: slide FMACN 7702.

Figure 5 Globorotalia crotonensis Conato and Follador, $\times 64$; Sample 251A-1-2, 40-42 cm; hypotype: slide FMACN 7702.

Figure 6 Globorotalia crotonensis Conato and Follador, $\times 56$; Sample 251A-4, CC; hypotype: slide FMACN 7702.

Figure 7 Globorotalia crotonensis Conato and Follador, $\times 64$; Sample 251A-4, CC; hypotype: slide FMACN 7702.

Figure $8 \quad$ Globorotalia limbata (Fornasini), $\times 48$; Sample 253-2-1, 50-52 cm; hypotype: slide FMACN 7703.

Figure $9 \quad$ Globorotalia limbata (Fornasini), $\times 72$; Sample 253-3-2, 40-42 cm; hypotype: slide FMACN 7703.

Figure $10 \quad$ Globorotalia limbata (Fornasini), $\times 48$; Sample 253-3-2, 40-42 cm; hypotype: slide FMACN 7703.

Figure $11 \quad$ Globorotalia limbata (Fornasini), $\times 48$; Sample 253-5, CC; hypotype: slide FMACN 7703.

Figure 12 Globorotalia limbata (Fornasini), $\times 48$; Sample 253-2-6, 40-42 cm; hypotype: slide FMACN 7703.

Figure 13 Globorotalia limbata (Fornasini), $\times 48$; Sample 253-2-5, 40-42 cm; hypotype: slide FMACN 7703.

Figure 14 Globorotalia limbata (Fornasini), $\times 48$; Sample 253-1, CC; hypotype: slide FMACN 7703.

Figure $15 \quad$ Globorotalia limbata (Fornasini), $\times 50$; Sample 253-2-4, 40-42 cm; hypotype: slide FMACN 7703.

Figure $16 \quad$ Globorotalia limbata (Fornasini), $\times 52$; Sample 253-3-2, 40-42 cm; hypotype: slide FMACN 7703. 
PLATE 10
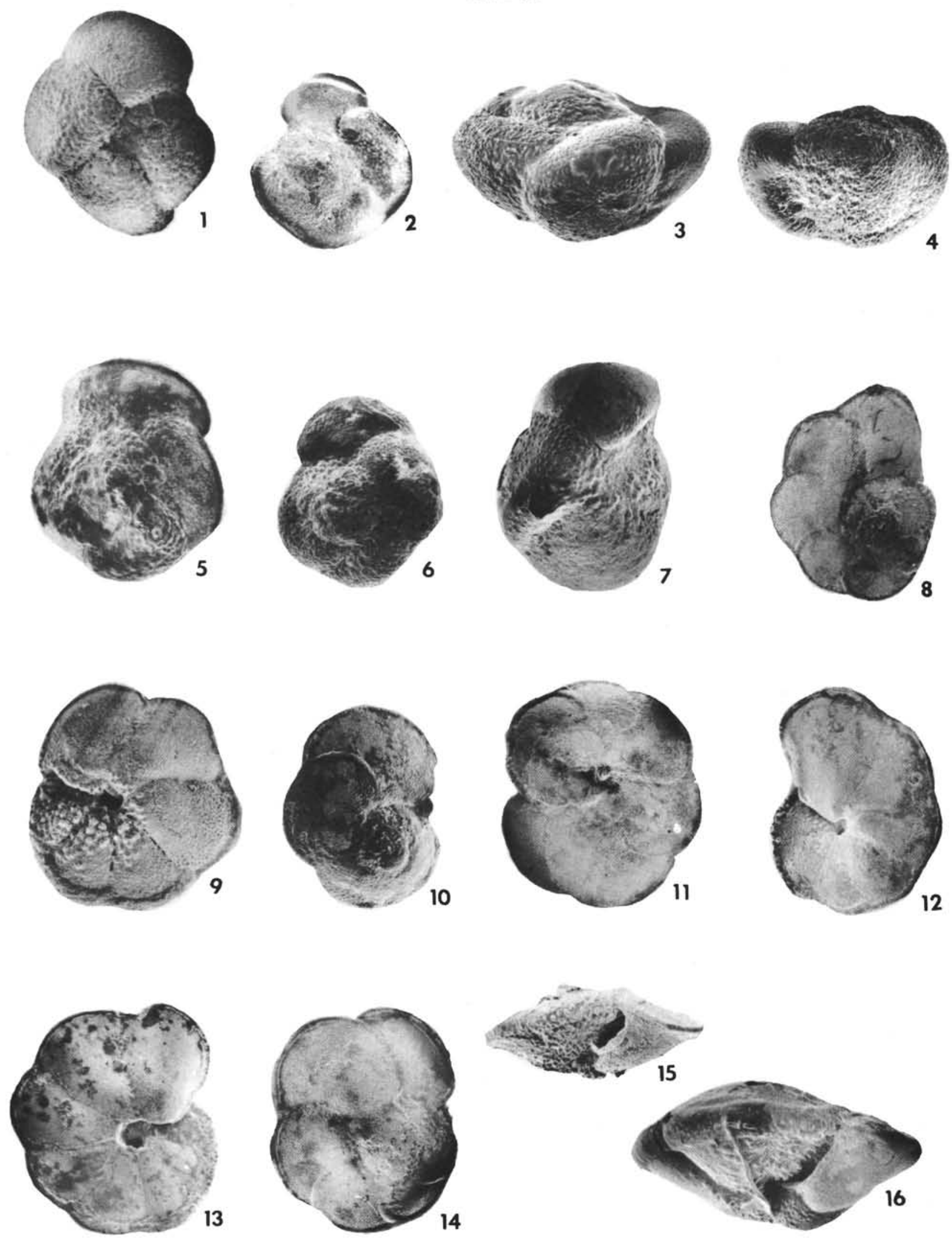
PLATE 11

Figure 1

Figure 2

Figure 3

Figure 4

Figure 5

Figure 6

Figure 7

Figure 8

Figure 9

Figure 10

Figure 11

Figure 12

Figure 13

Figure 14

Figure 15

Figure 16

Figure 17

Figure 18
Globorotalia limbata (Fornasini), $\times 48$; Sample 253-2, CC; hypotype: slide FMACN 7703.

Globorotalia aff. limbata (Fornasini), $\times 60$; Sample 254-8-2, 40-42 cm; hypotype: slide FMACN 7704.

Globorotalia aff. limbata (Fornasini), $\times 68$; Sample 254-8-2, 115-117 cm; hypotype: slide FMACN 7704.

Globorotalia aff. limbata (Fornasini), $\times 72$; Sample 254-7, CC; hypotype: slide FMACN 7704.

Globorotalia miozea conoidea Walters, $\times 48$; Sample 254-3-2, 115-117 cm; hypotype: slide FMACN 7705.

Globorotalia miozea conoidea Walters, $\times 60$; Sample 251A-5-1, 40-42 cm; hypotype: slide FMACN 7705.

Globorotalia miozea conoidea Walters, $\times 68$; Sample 251A-10, CC; hypotype: slide FMACN 7705.

Globorotalia miozea conoidea Walters, $\times 56$; Sample 254-3-2, 115-117 cm; hypotype: slide FMACN 7705.

Globorotalia miozea conoidea Walters, $\times 40$; Sample 254-3-2, 115-117 cm; hypotype: slide FMACN 7705.

Globorotalia miozea conoidea Walters, $\times 40$; Sample 254-2-6, 115-117 cm; hypotype: slide FMACN 7705.

Globorotalia miozea conoidea Walters, $\times 40$; Sample 254-2-6, 115-117 cm; hypotype: slide FMACN 7705.

Globorotalia miozea conoidea Walters, $\times 64$; Sample 251A-5-1, 40-42 cm; hypotype: slide FMACN 7705.

Globorotalia miozea conoidea Walters, $\times 64$; Sample 254-2-6, 115-117 cm; hypotype: slide FMACN 7705.

Globorotalia miozea conoidea Walters, $\times 64$; Sample 254-3-2, 115-117 cm; hypotype: slide FMACN 7705.

Globorotalia miozea conoidea Walters, $\times 60$; Sample 254-3-2, 115-117 cm; hypotype: slide FMACN 7705.

Globorotalia miozea conoidea Walters, $\times 60$; Sample 254-3-2, 40-42 cm; hypotype: slide FMACN 7705 .

Globorotalia miozea conoidea Walters, $\times 48$; Sample 254-3-2, 40-42 cm; hypotype: slide FMACN 7705 .

Globorotalia miozea conoidea Walters, $\times 60$; Sample 254-3-2, 40-42 cm; hypotype: slide FMACN 7705 . 
PLATE 11
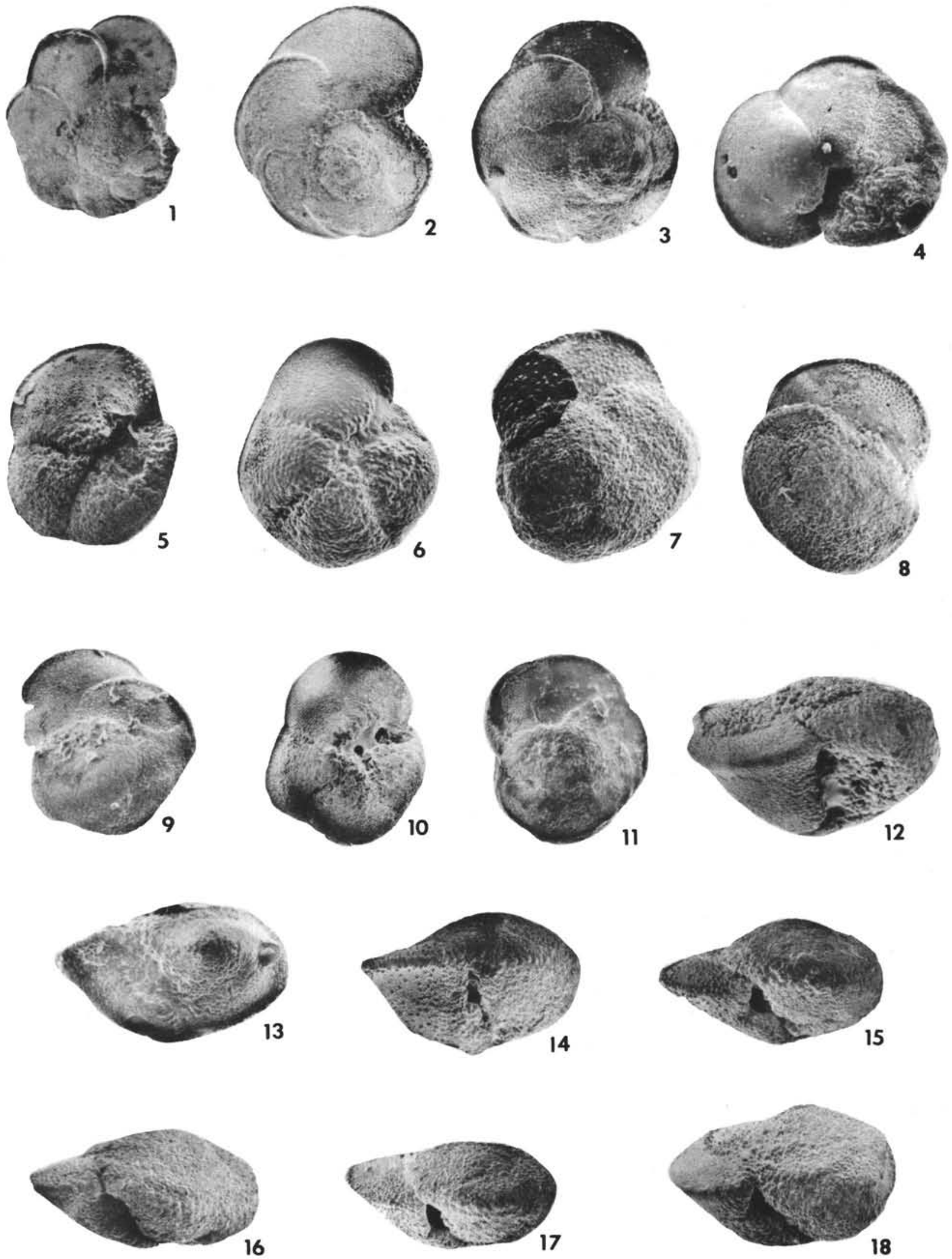


\section{PLATE 12}

Figure 1 ?Globorotalia miocenica Palmer, $\times 48$; Sample 25410, CC; hypotype: slide FMACN 7706.

Figure 2 ?Globorotalia miocenica Palmer, $\times 80$; Sample 25410-6, 115-117 cm; hypotype: slide FMACN 7706.

Figure 3

?Globorotalia miocenica Palmer, $\times 64$; Sample 2549-3, 115-117 cm; hypotype: slide FMACN 7706.

Figure 4 Globorotalia opima Bolli, s.l. $\times 80$; Sample 253-9-2, 24-26 cm; hypotype: slide FMACN 7707.

Figure $5 \quad$ Globorotalia opima Bolli, s.l., $\times 92$; Sample 254-19, CC; hypotype: slide FMACN 7707.

Figure 6 Globorotalia opima Bolli, s.l., ×80; Sample 254-192, 115-117 cm; hypotype: slide FMACN 7707.

Figure 7

Globorotalia opima Bolli, s.l., $\times 100$; Sample 25420, CC; hypotype: slide FMACN 7707.

Figure $8 \quad$ Globorotalia opima Bolli, s.l., $\times 80$; Sample 254-183, 125-127 cm; hypotype: slide FMACN 7707.

Figure 9

Globorotalia peripheroacuta Blow and Banner, $\times 92$; Sample 253-7, CC; hypotype: slide FMACN 7708 .

Figure 10 Globorotalia peripheroacuta Blow and Banner, $\times 120$; Sample 253-8-2, 40-42 cm; hypotype: slide FMACN 7708.

Figure 11 Globorotalia peripheroacuta Blow and Banner, $\times 92$; Sample 253-8-2, 40-42 cm; hypotype: slide FMACN 7708.

Figure 12 ?Globorotalia pseudomiocenica Bolli and Bermúdez, $\times 80$; Sample 254-12-1, 40-42 cm; hypotype: slide FMACN 7710.

Figure 13 Globorotalia peripheroronda Blow and Banner, $\times 100$; Sample 254-11-3, 40-42 cm; hypotype: slide FMACN 7709.

Figure 14 Globorotalia peripheroronda Blow and Banner, $\times 112$; Sample 254-11-4, 115-117 cm; hypotype: slide FMACN 7709.

Figure 15 ?Globorotalia pseudomiocenica Bolli and Bermúdez, ×80; Sample 254-12-1, 40-42 cm; hypotype: slide FMACN 7710.

Figure 16 ?Globorotalia pseudomiocenica Bolli and Bermúdez, ×76; Sample 254-12-3, 141-143 cm; hypotype: slide FMACN 7710. 
PLATE 12
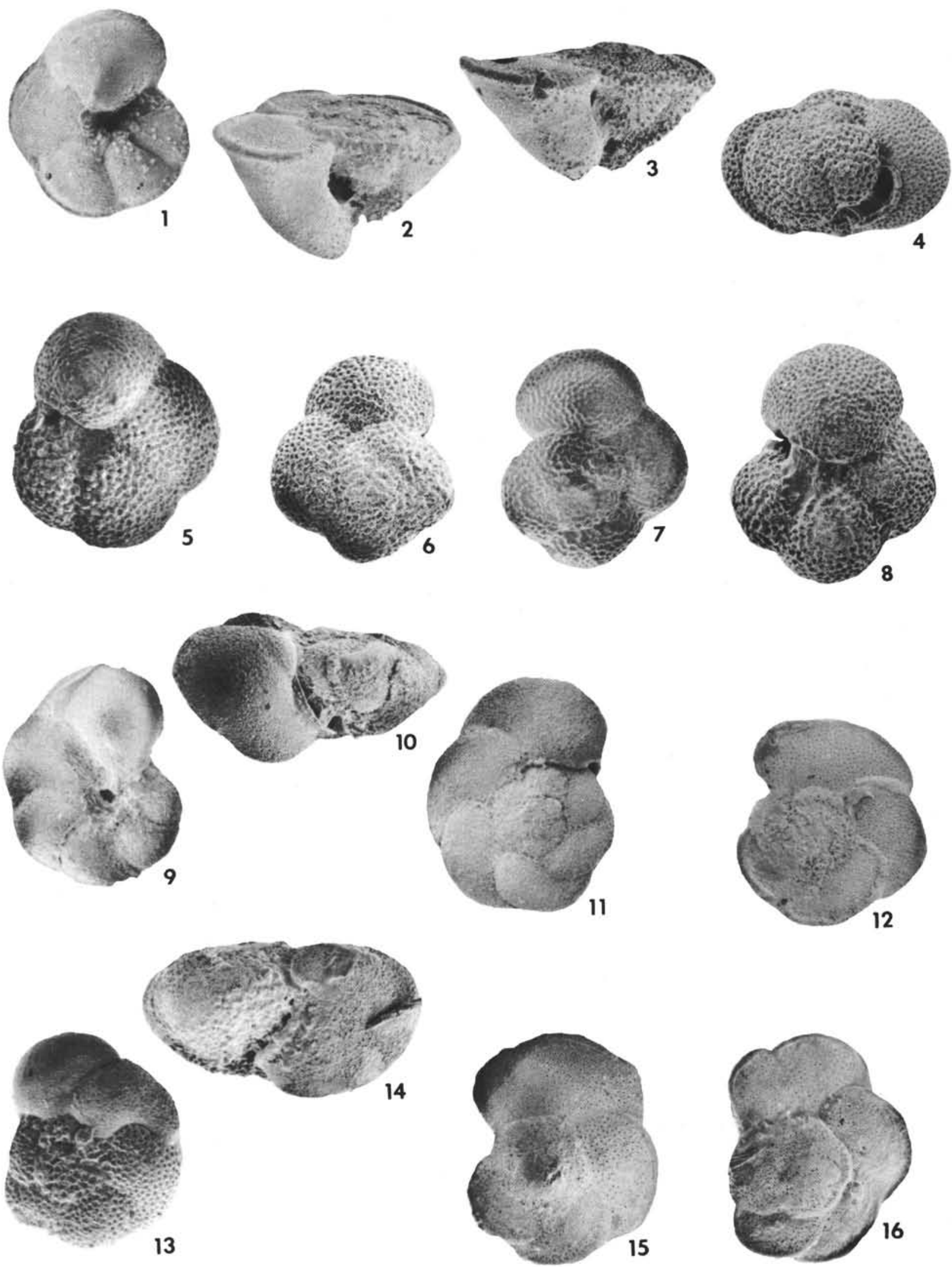


\section{PLATE 13}

Figure $1 \quad$ Globorotalia scitula (Brady), $\times 80$; Sample 258A-11, 61-63 cm; hypotype: slide FMACN 7711 .

Figure 2 Globorotalia pseudobulloides (Plummer), $\times 80$; Sample 253-11-2, 40-42 cm; hypotype: slide FMACN 7712.

Figure 3 Globorotalia pseudobulloides (Plummer), $\times 92$; Sample 253-12-5, 40-42 cm; hypotype: slide FMACN 7712.

Figure $4 \quad$ Globorotalia cf. pseudopima Blow, $\times 80$; Sample 254-1, CC; hypotype: slide FMACN 7713.

Figure $5 \quad$ Globorotalia tosaensis Takayanagi and Saito, $\times 80$; Sample 254-2-5, 40-42 cm; hypotype: slide FMACN 7714.

Figure 6

Globorotalia tosaensis Takayanagi and Saito, $\times 80$; Sample 254-2-5, 40-42 cm; hypotype: slide FMACN 7714.

Figure $7 \quad$ Globorotalia tosaensis Takayanagi and Saito, $\times 52$; Sample 254-2-5, 40-42 cm; hypotype: slide FMACN 7714.

Figure $8 \quad$ Orbulina suturalis Brönnimann, $\times 160$; Sample 251A-14-1, 10-12 cm; hypotype: slide FMACN 7715 .

Figure $9 \quad$ Globorotalia truncatulinoides (d'Orbigny), $\times 64$; Sample 254-1-2, 115-117 cm; hypotype: slide FMACN 7716.

Figure $10 \quad$ Globorotalia truncatulinoides (d'Orbigny), $\times 64$; Sample 254-1-2, 115-117 cm; hypotype: slide FMACN 7716.

Figure $11 \quad$ Globorotalia truncatulinoides (d'Orbigny), $\times 64$; Sample 254-1-2, 115-117 cm; hypotype: slide FMACN 7716.

Figure $12 \quad$ Sphaeroidinella subdehiscens Blow, $\times 56$; Sample 254-3-4, 115-117 cm; hypotype: slide FMACN 7719.

Figure 13 Pulleniatina obliquiloculata praecursor Banner and Blow, $\times 80$; Sample 258-3 CC; hypotype: slide FMACN 7717.

Figure 14 Sphaeroidinella seminulina (Schwager), $\times 48$; Sample 254-2-5, 40-42 cm; hypotype: slide FMACN 7718.

Figure 15 Sphaeroidinella dehiscens (Parker and Jones), $\times 44$; Sample 254-1-2, 115-117 cm; hypotype: slide FMACN 7720.

Figure 16 Sphaeroidinella seminulina (Schwager), $\times 64$; Sample 254-2-5, 40-42 cm; hypotype: slide FMACN 7718.

Figure 17 Sphaeroidinella seminulina (Schwager), $\times 54$; Sample 254-2-5, 40-42 cm; hypotype: slide FMACN 7718. 
PLATE 13
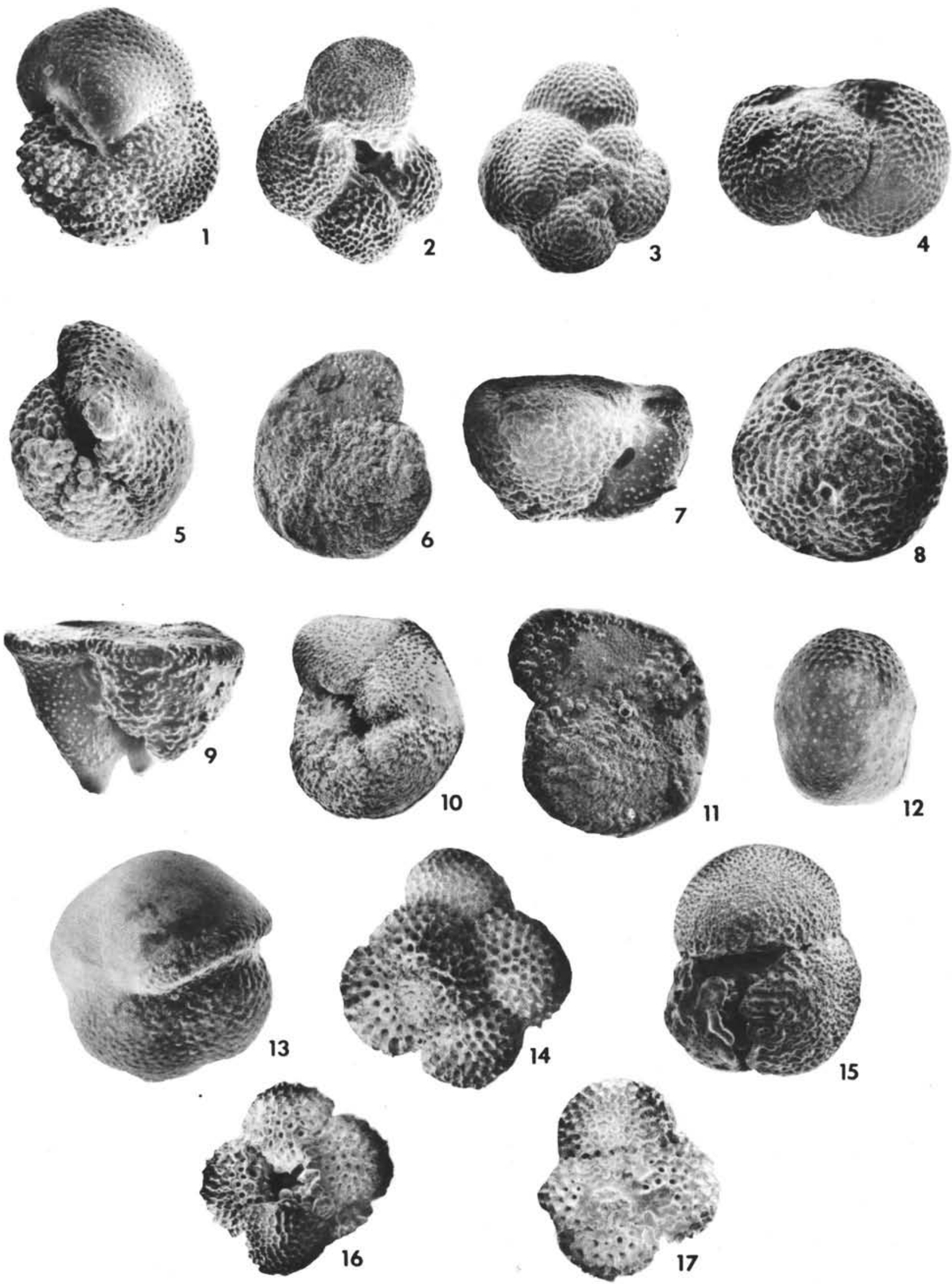


\section{PLATE 14}

Figure la-b Globorotalia petaliformis n. sp., holospecietypus, $\times 48$; Sample 254-10, CC; slide FMACN 7646.

Figures 2-16 Globorotalia petaliformis n. sp., paratypi; slide FMACN 7648

2. $\times 40$; Sample 254-12, CC

3. $\times 48$; Sample 254-11-2, 115-117 cm

4. $\times 48$; Sample 254-11-2, $115-117 \mathrm{~cm}$

5. $\times 56$; Sample $254-10$, CC

6. $\times 48$; Sample 255-4-1, $40-42 \mathrm{~cm}$

7. $\times 48$; Sample $253-7-3,40-42 \mathrm{~cm}$

8. $\times 48$; Sample 254-10-5, $115-117 \mathrm{~cm}$

9. $\times 56$; Sample 254-10-6, $40-42 \mathrm{~cm}$

10. $\times 64$; Sample 254-10-4, 40-42 cm

11. $\times 56$; Sample $254-10-4,40-42 \mathrm{~cm}$

12. $\times 76$; Sample 254-11-4, $115-117 \mathrm{~cm}$

13. $\times 60$; Sample 254-11-5, 115-117 cm

14. $\times 56$; Sample 254-11, CC

15. $\times 40$; Sample 254-12, CC

16. $\times 52$; Sample 254-10, CC 
PLATE 14
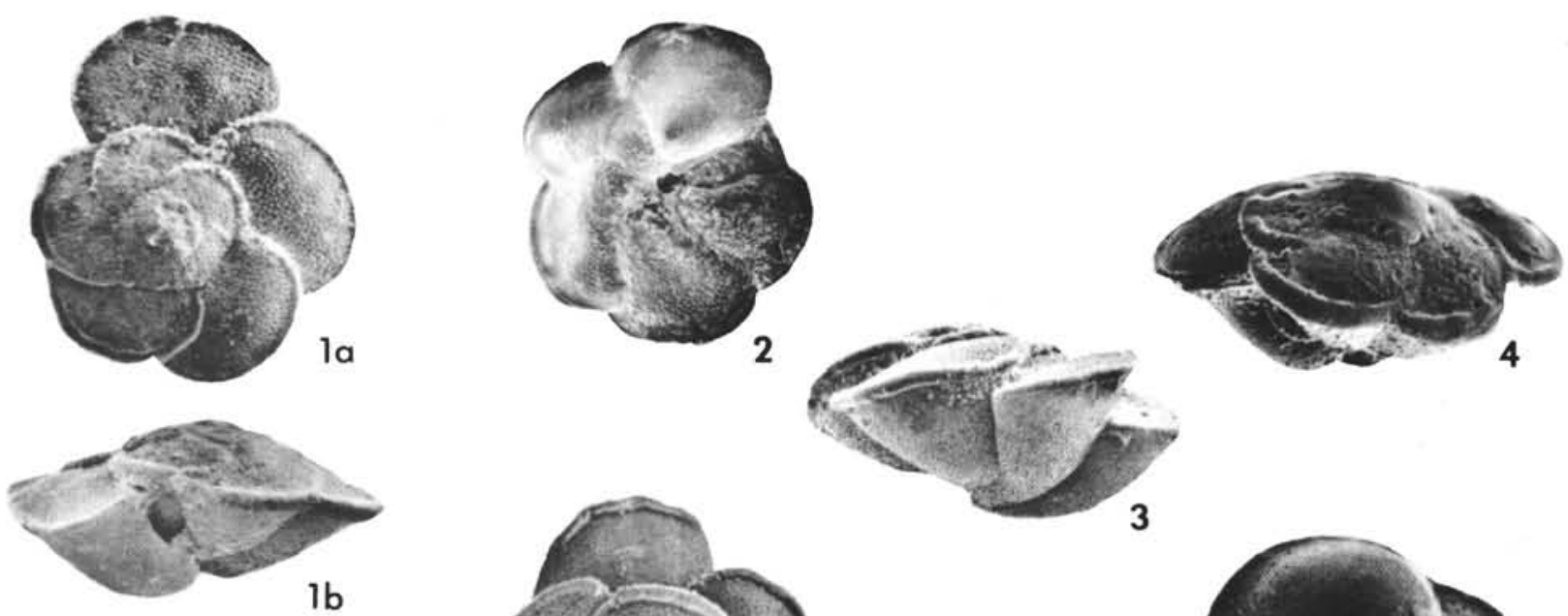

Ib
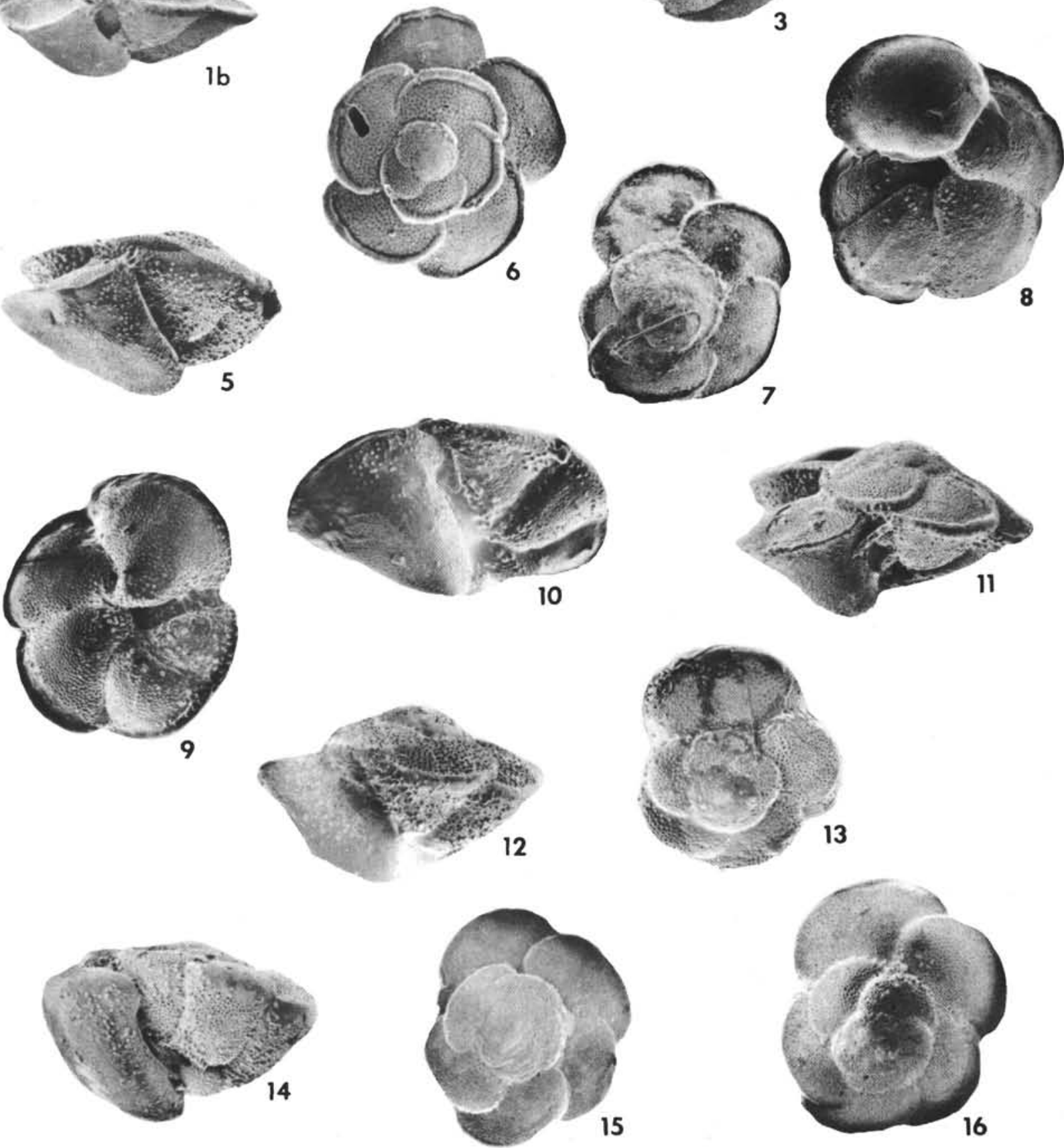